MÉMOIRES DE LA SMF 95

\title{
ERGODICITÉ ET ÉQUIDISTRIBUTION EN COURBURE NÉGATIVE
}

Thomas Roblin

Société Mathématique de France 2003

Publié avec le concours du Centre National de la Recherche Scientifique 
T. Roblin

Laboratoire de Probabilités et Modèles Aléatoires, UMR CNRS 7599,

Université de Paris VI, 4, place Jussieu, 75252 Paris Cedex 05.

E-mail : roblin@ccr.jussieu.fr

Classification mathématique par sujets (2000). - 37D40, 37F35.

Mots clefs. - Groupes discrets, courbure négative, dénombrement asymptotique, densités conformes, théorie ergodique, flot géodésique, feuilletage horosphérique, unique ergodicité, géodésiques fermées. 


\title{
ERGODICITÉ ET ÉQUIDISTRIBUTION EN COURBURE NÉGATIVE
}

\author{
Thomas Roblin
}

Résumé. - Considérant un groupe d'isométries discret agissant sur un espace CAT $(-1)$, nous établissons successivement, par des méthodes nouvelles et élémentaires, un théorème d'ergodicité du feuilletage horosphérique associé, le mélange du flot géodésique, l'équidistribution des points orbitaux du groupe, avec premier terme asymptotique exponentiel de la fonction orbitale, l'équidistribution des géodésiques fermées primitives avec, dans le cas géométriquement fini, leur dénombrement asymptotique. Enfin, nous démontrons un théorème général d'unique ergodicité du feuilletage horosphérique pour les groupes à mesure de Bowen-Margulis-Sullivan finie. Ces divers résultats sont nouveaux dans leur généralité.

\section{Abstract (Ergodicity and equidistribution in negative curvature)}

We consider a discrete isometry group acting on a CAT $(-1)$ space, and successively establish, by new and elementary methods, an ergodicity theorem for the associated horospherical foliation, then mixing of the geodesic flow, orbital equidistribution of the group, with first asymptotic for the orbital counting function, equidistribution of primitive closed geodesics with, in the geometrically finite case, asymptotic counting. Endly, we prove a general unique ergodicity theorem for the horospherical foliation for groups with finite Bowen-Margulis-Sullivan measure. Those various results are new in their generality. 



\section{TABLE DES MATIÈRES}

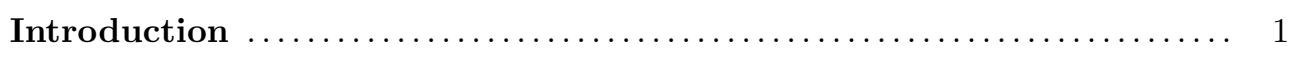

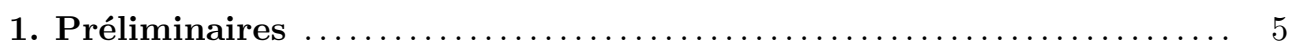

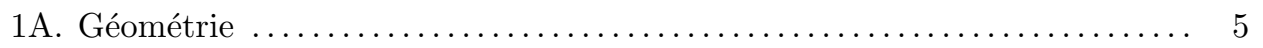

1B. Densités conformes et invariantes à l'infini $\ldots \ldots \ldots \ldots \ldots \ldots \ldots \ldots \ldots$

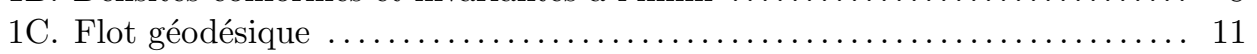

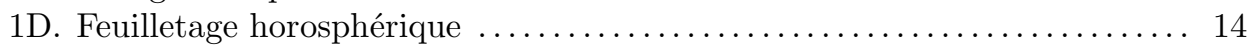

1E. Ergodicité du flot géodésique et groupes de type divergent $\ldots \ldots \ldots \ldots \ldots 17$

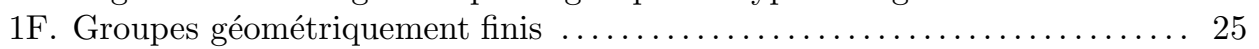

1G. Distances et mesures sur les horosphères $\ldots \ldots \ldots \ldots \ldots \ldots \ldots \ldots \ldots \ldots$

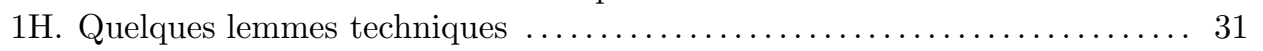

2. Ergodicité du feuilletage horosphérique $\ldots \ldots \ldots \ldots \ldots \ldots \ldots \ldots \ldots$

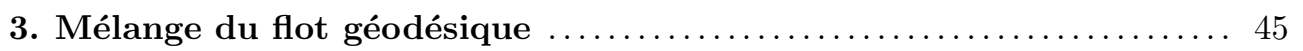

4. Dénombrement et équidistribution asymptotique des orbites . . . . 55

5. Équidistribution asymptotique des géodésiques fermées primitives 67

6. Moyennes horosphériques et classification des mesures invariantes . 77

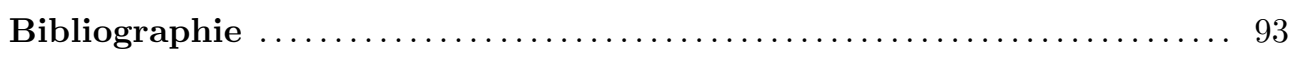





\section{INTRODUCTION}

Dans le présent travail, nous nous sommes proposé d'établir des résultats généraux concernant la théorie ergodique du feuilletage horosphérique (ergodicité, unique ergodicité), le flot géodésique (mélange), le dénombrement et l'équidistribution asymptotiques des orbites du groupe d'isométries discret associé, ainsi que des géodésiques fermées. Signalons d'emblée que tous nos résultats étaient déjà connus dans le cadre des surfaces riemanniennes compactes de courbure négative constante, de bien des manières et parfois depuis longtemps. Aussi nous sommes-nous plutôt attachés ici à étendre autant que possible ces résultats dans le contexte de la géométrie de courbure négative, ce qui nous impose le recours exclusif à des méthodes élémentaires. C'est ainsi que notre environnement sera celui des espaces CAT $(-1)$, large famille comprenant les variétés riemanniennes complètes simplement connexes à courbures sectionnelles au plus -1 , mais aussi les arbres, où nous étudierons l'action d'un groupe $\Gamma$ d'isométries discret non élémentaire quelconque. Il se trouve qu'il s'agit là du cadre axiomatique naturel où puisse s'échafauder la théorie des densités conformes introduite par Patterson et Sullivan, laquelle sera présente tout au long de ce travail, car nous verrons qu'elle est suscitée par la nature même des problèmes envisagés (à propos des mesures invariantes pour les systèmes dynamiques en jeu, mais aussi des mesures d'équidistribution). C'est de cette manière que l'on peut encore aborder avec fruit les situations où le groupe discret en question n'agit pas de façon cocompacte.

Ce qui aura permis les généralisations que nous proposons ici, c'est la nature élémentaire des méthodes employées. D'ailleurs, pour qui connaît la géométrie de courbure négative, tout au moins le demi-plan de Poincaré, le présent article se suffit à lui-même, car nous ne nous sommes adressés à aucune des théories d'une certaine manière extrinsèques (théorie spectrale, dynamique symbolique) qui ont été bien souvent utilisées jusqu'à présent, non sans succès, mais qui ne peuvent toutefois porter dans leur généralité les résultats visés ici. 
Si l'ensemble des thèmes abordés risque de paraître quelque peu disparate de prime abord, on verra qu'il n'en est rien, puisque ces divers problèmes seront reliés et traités les uns à partir des autres, selon un enchaînement que nous allons maintenant décrire, en suivant le déroulement de l'exposé subséquent. Cela justifie à notre sens la collection de ces résultats en un seul et même article.

Par feuilletage horosphérique, nous entendrons ici simplement l'ensemble des horosphères, ou encore le produit du bord à l'infini et de $\mathbb{R}$. Le système habituellement considéré, flot horocyclique ou feuilletage horosphérique stable ou instable, se ramène à l'action de $\Gamma$ sur le précédent, avec ses mesures invariantes. La pierre angulaire de ce travail est un théorème d'ergodicité du feuilletage horosphérique, qui énonce que sous certaines conditions, les actions de $\Gamma$, d'une part sur le bord à l'infini par rapport à une densité conforme invariante, et d'autre part sur le feuilletage horosphérique par rapport à une mesure invariante canoniquement associée à la précédente densité, sont simultanément ergodiques (théorème 2.2). Ce résultat, qui rejoint des travaux récents de V.Kaimanovich, ne sera pas ici utilisé dans toute son étendue, loin s'en faut, puisque nous nous contenterons d'en extraire un corollaire affirmant l'ergodicité $\mathrm{du}$ feuilletage horosphérique lorsque $\Gamma$ est de type divergent, c'est-à-dire lorsque le flot géodésique est ergodique (corollaire 2.3) ; la seule condition requise - et que l'on sait nécessaire -, est que le spectre des longueurs de $\Gamma$ ne soit pas arithmétique, hypothèse que l'on conservera pour tout le restant de cet article (à noter qu'elle est toujours acquise en courbure constante et dans quelques autres cas).

Ce dernier corollaire nous permettra d'établir brièvement, pour n'importe quel groupe $\Gamma$, la propriété de mélange du flot géodésique pour la mesure de BowenMargulis-Sullivan, que cette dernière soit finie ou non (théorème 3.1). Ce résultat, qui vient d'être encore démontré différemment par M. Babillot, n'était auparavant pas connu dans cette généralité, y compris en mesure finie. Il constitue véritablement la cheville ouvrière de notre travail, attendu que tout le restant en découle ; ses applications se répartissent en deux branches indépendantes : d'une part les divers théorèmes d'équidistribution asymptotique des chapitres 4 et 5, d'autre part celui d'unique ergodicité du feuilletage horosphérique du chapitre final (que le lecteur pourra aborder sitôt qu'il aura pris connaissance du corollaire 3.2 du théorème 3.1).

Dans le chapitre 4 , le problème de la répartition asymptotique des points d'une orbite de $\Gamma$ dans l'espace ( « revêtement universel ») sur lequel il agit, est ici relié géométriquement au phénomène de mélange du flot géodésique, ce qui conduit à un énoncé d'équidistribution orbitale (théorème 4.1) exactement équivalent au théorème 3.1. Il s'organise de façon dichotomique, en distinguant le cas où $\Gamma$ admet une mesure de Bowen-Margulis-Sullivan finie (théorème 4.1.1), et le cas contraire (théorème 4.1.2). Dans le premier cas, on y voit que la fonction orbitale de $\Gamma$ est exactement équivalente à une exponentielle (corollaire 2); au surplus, la probabilité uniforme sur les points d'une orbite dans une boule de rayon croissant (ce que dénombre justement la 
fonction orbitale) converge vaguement vers la mesure conforme de Patterson-Sullivan (corollaire 1); mais c'est un énoncé encore plus général qui traduit le mélange du flot géodésique, et qui consiste en une équidistribution de certaines paires de points orbitaux (théorème 4.1.1) - ce qui sera fondamental pour établir les résultats du chapitre 5. Dans le second cas, la signification du mélange du flot géodésique se résume en le fait que la fonction orbitale est négligeable devant l'exponentielle précédente (théorème 4.1.2). Quoique sur ce sujet de très nombreux résultats partiels fussent déjà connus, certains même plus poussés, aucun d'entre eux ne couvrait complètement la question du premier terme exponentiel asymptotique de la fonction orbitale au sens de la dichotomie précédente; en fait, l'équivalent exponentiel n'était jusqu'à présent même pas établi pour tous les groupes géométriquement finis en courbure constante, et la contrepartie que constitue le théorème 4.1.2 n'était pratiquement jamais apparue. En outre, un fait important mérite d'être noté : c'est que les groupes géométriquement finis sont très loin d'être les seuls à admettre une mesure de Bowen-Margulis-Sullivan finie, comme l'ont montré récemment A. Ancona et M. Peigné; aussi le véritable critère dichotomique pour le comportement exponentiel de la fonction orbitale n'est pas la finitude géométrique, contrairement à ce que l'on a pu soupçonner. Pour terminer, nous insisterons sur le fait que les méthodes jusque-là employées pour aborder ces questions n'étaient que rarement élémentaires (théorie spectrale, dynamique symbolique).

Le chapitre 5 traite de la même façon le problème du comportement asymptotique des géodésiques fermées primitives, avec un théorème d'équidistribution (théorème 5.1), lui aussi exact équivalent des énoncés 3.1 et 4.1 , et que l'on tirera de ce dernier. Lorsque $\Gamma$ est convexe-cocompact, il en ressort un équivalent asymptotique du nombre de géodésiques fermées primitives de longueur majorée par un nombre croissant; or ce dénombrement asymptotique reste valide dans le cas où $\Gamma$ est seulement supposé géométriquement fini, grâce à des arguments supplémentaires (théorème 5.2). Les mêmes commentaires que précédemment ont cours ici, et nous ne les répétons pas.

Enfin, le chapitre 6 revient au feuilletage horosphérique, exclusivement pour un groupe $\Gamma$ admettant une mesure de Bowen-Margulis-Sullivan finie. Le théorème principal est le 6.4, qui affirme que le feuilletage horosphérique, restreint au produit de l'ensemble limite conique de $\Gamma$ (qui correspond aux points non errants du flot géodésique) et de $\mathbb{R}$, est uniquement ergodique. Pour $\Gamma$ géométriquement fini, il en découle une classification complète des mesures invariantes par le feuilletage horosphérique (corollaire 6.5). Ainsi a-t-on pu, par une méthode élémentaire, englober les résultats déjà connus, qui portaient soit sur les groupes convexe-cocompacts, soit sur les probabilités invariantes par le flot horocyclique en courbure constante. L'objet fondamental dont nous usons est une certaine moyenne horosphérique, succédané des moyennes ergodiques classiques. 
Au cours de cette présentation de la colonne vertébrale de l'article, nous avons laissé de côté quelques résultats. Nous signalerons ici seulement un résultat général d'équidistribution asymptotique des orbites des points paraboliques (théorème 4.2), et aussi un énoncé ergodique de convergence des moyennes horosphériques (théorème 6.1).

Nous avons jugé utile que cet article débutât par des préliminaires, dont l'extension a toutefois ceci de fâcheux que l'exposé s'en trouve considérablement allongé, mais nous croyons pouvoir nous en justifier. En effet, quelques concepts usuels de la géométrie hyperbolique n'avaient pas encore fait l'objet d'une définition et d'une étude dans le cadre des espaces CAT $(-1)$. De plus, certains résultats fondamentaux n'avaient pas été établis dans le cadre des espaces CAT $(-1)$; lorsque la généralisation allait de soi, comme il arrive assez souvent, nous nous sommes contentés d'énoncés ; mais un théorème important, connu sous le nom de Hopf-Tsuji-Sullivan (théorème 1.7), gardait quelque obscurité, ce jusqu'en courbure constante, du fait notamment que certaines preuves présentaient des incorrections ; aussi avons-nous tenu à l'éclaircir une fois pour toutes en en exposant une preuve, afin de ne pas saper l'ensemble. Enfin, l'on pourra se dispenser en première lecture de la plus grande partie de ces préliminaires (voir les indications données au commencement).

Nous tenons à exprimer notre gratitude envers feu M. Babillot pour son attention portée au manuscrit. Nous en remercions également le rapporteur. 


\section{CHAPITRE 1}

\section{PRÉLIMINAIRES}

Cette première partie esquisse une introduction complète au sujet traité, la seule connaissance du disque hyperbolique (ou demi-plan de Poincaré) restant requise. Si une bonne part des notions et résultats présentés dans les sections $1 \mathrm{~A}$ à $1 \mathrm{~F}$ sont bien connus dans le cadre des espaces hyperboliques, voire dans celui des variétés à courbure pincée, et parfois même déjà dans celui des espaces CAT $(-1)$, certaines généralisations, au demeurant naturelles, ne semblent pas avoir été jusque-là sûrement examinées. Nous nous sommes ici efforcés de donner au lecteur, dans la mesure du possible, les éléments nécessaires afin qu'il puisse reconstruire aisément la théorie sur laquelle notre travail est échafaudé. Nous avons cru bon de rappeler les résultats fondamentaux incontournables pour la suite, et d'insister sur leur enchaînement logique. Nous avons aussi tenu à détailler les points de démonstrations qui nous ont paru insuffisamment éclaircis dans la littérature courante sur le sujet.

Avant d'entamer les parties 2 à 6 , qui constituent le principal apport de ce travail, il n'est généralement point besoin de lire les préliminaires dans leur ensemble. Ils sont organisés de telle sorte qu'il suffit de s'imprégner du tronc commun que forment les sections $1 \mathrm{~A}$ à $1 \mathrm{E}$ (on pourra omettre la preuve étendue du théorème 1.7 en première lecture) pour se trouver déjà en mesure d'aborder le chapitre 2 puis les théorèmes $3.1,4.1$ et 5.1 . Le théorème 5.2 requiert d'avoir pris connaissance de la section $1 \mathrm{~F}$, dont la matière n'est au surplus pas nouvelle en courbure pincée. Quant au reste, l'accès en est commandé par les sections $1 \mathrm{G}$ pour les concepts, et $1 \mathrm{H}$ pour les lemmes techniques.

\section{A. Géométrie}

Tout au long du présent article, nous considérerons un espace métrique $\mathrm{CAT}(-1)$ propre $X$. Pour tout ce qui a trait à ces espaces, nous nous référons à $[\mathbf{B o u}]$ et $[\mathbf{G}-\mathbf{H}]$. Afin d'alléger la notation de la distance entre deux points $x$ et $y$ dans $X$, nous ferons 
l'économie d'un symbole et la désignerons simplement par $(x, y)$, ce qui, espérons-le, ne sera pas cause de confusions. Rappelons que par définition, $X$ est un espace métrique, propre (complet et localement compact), géodésique (deux points quelconques sont reliés par un chemin minimisant la longueur), satisfaisant à la propriété dite $\mathrm{CAT}(-1)$ (par référence au théorème de comparaison d'Aleksandrov-Toponogov en géométrie riemannienne), que nous allons formuler. Étant donné un triangle géodésique $(a, b, c)$ dans $X$, il existe une application $x \mapsto \bar{x}$ de $(a, b, c)$ sur un triangle $(\bar{a}, \bar{b}, \bar{c})$ du demi-plan hyperbolique qui induit sur chaque côté de $(a, b, c)$ une isométrie sur le côté correspondant de $(\bar{a}, \bar{b}, \bar{c})$; ce dernier triangle est unique à isométrie près, et est appelé triangle de comparaison. La propriété $\mathrm{CAT}(-1)$ spécifie que pour tout triangle $(a, b, c)$ dans $X$, l'application $x \mapsto \bar{x}$ de $(a, b, c)$ sur son triangle de comparaison est contractante, c'est-à-dire que $(x, y)$ est inférieure ou égale à la distance de $\bar{x}$ à $\bar{y}$ dans le demi-plan hyperbolique pour tous $x$ et $y$ sur le triangle $(a, b, c)$. Outre sa grande souplesse - appréciation que ce travail ne fera que corroborer -, la définition précédente permet d'englober un large éventail d'espaces dont le caractère est de posséder une courbure majorée en deçà de zéro. Parmi les espaces CAT $(-1)$, on regroupe notamment les espaces hyperboliques (de toute dimension), plus généralement les variétés riemanniennes complètes simplement connexes à courbures sectionnelles au plus -1 , et aussi les arbres et les immeubles hyperboliques.

Tout autant que pour le disque hyperbolique, on peut construire un bord à l'infini pour $X$ selon le procédé suivant. Un rayon géodésique est une isométrie d'un intervalle maximal $I$ d'origine 0 de $\mathbb{R}$ dans $X$; nécessairement $I=[0,+\infty)$ ou bien $I=[0, a]$ $\left(a \in \mathbb{R}^{+}\right)$, auquel cas nous convenons présentement de prolonger ce rayon à $[0,+\infty)$ en lui assignant une valeur constante sur $[a,+\infty)$. Deux rayons sont dits asymptotes si la distance entre leurs images à chaque instant est uniformément bornée. Le bord à l'infini $\partial X$ de $X$ (dit aussi bord visuel) est défini comme étant l'ensemble des classes de rayons géodésiques asymptotes. En voyant les points de $X$ comme extrémités des segments géodésiques d'origine fixe, on peut munir l'ensemble $\bar{X}=X \cup \partial X$ d'une topologie naturelle (convergence compacte), qui en fait une compactification de $X$ dans laquelle $X$ est un ouvert dense. Signalons que l'on confond habituellement une géodésique (isométrie d'un intervalle maximal de $\mathbb{R}$ dans $X$ ) avec son image. Par deux points distincts $a$ et $b$ quelconques dans $\bar{X}$ passe une unique géodésique que nous noterons $(a b)$. Quant au rayon géodésique issu de $a \in X$ et passant par $b \in X$ ou finissant en $b \in \partial X$, il sera noté $] a b)$. Dans la généralité des espaces CAT $(-1)$, toutes les géodésiques ne sont pas infinies. Cependant, les rayons géodésiques finis ne joueront aucun rôle et seront de fait ignorés, pour la seule et bonne raison que notre attention sera tout entière tournée vers le bord à l'infini. D'autre part, les isométries de $X$ (dans lui-même) se prolongent en homéomorphismes de $\bar{X}$ dans lui-même, pour désigner lesquels nous conserverons en pratique les mêmes symboles. 
L'objet essentiel de la courbure négative sera ici la fonction de Busemann $\beta$. Pour $\xi \in \partial X$ et $x, y$ dans $X$, on peut définir $\beta_{\xi}(x, y)=\lim _{t \rightarrow+\infty}\left[\left(x, \xi_{t}\right)-\left(y, \xi_{t}\right)\right]$ pour un (tout) rayon géodésique $t \mapsto \xi_{t}$ finissant en $\xi$. Observons la propriété de cocycle $\beta_{\xi}(x, y)+\beta_{\xi}(y, z)=\beta_{\xi}(x, z)$, ainsi que l'invariance par isométrie $\beta_{\phi \xi}(\phi x, \phi y)=$ $\beta_{\xi}(x, y)$ (dans toute cette introduction, $\phi$ désignera une isométrie de $X$ quelconque). Notons aussi l'inégalité (d'origine triangulaire) $\left|\beta_{\xi}(x, y)\right| \leqslant(x, y)$. Il est prouvé la continuité de la fonction de $\bar{X} \times X^{2}$ dans $\mathbb{R}$ qui à $(a, x, y) \in X^{3}$ associe $(x, a)-(y, a)$, et à $(\xi, x, y) \in \partial X \times X^{2}, \beta_{\xi}(x, y)$.

Une horosphère dans $X$ est une «ligne de niveau » d'une fonction de Busemann $\beta_{\xi}(x,$.$) . Plus précisément, l'horosphère basée en \xi \in \partial X$ et passant par $x \in X$ est l'ensemble des $y \in X$ tels que $\beta_{\xi}(x, y)=0$. La fonction $\beta_{\xi}$ définit en fait une « distance horosphérique » (algébrique) vue du point base $\xi$. L'horoboule (ouverte) bordée par l'horosphère basée en $\xi \in \partial X$ et passant par $x \in X$ est l'ensemble des $y \in X$ tels que $\beta_{\xi}(x, y)>0$. On voit aisément (par comparaison) que les horoboules fermées sont strictement convexes. On vérifie aussi qu'en accord avec l'étymologie, la sphère de centre $a \in X$ passant par un point $x \in X$ fixé, tend, quand $a \rightarrow \xi \in \partial X$, vers l'horosphère basée en $\xi$ et contenant $x$.

On appelle produit de Gromov en $x \in X$ de deux points $a$ et $b$ dans $X$ la quantité $\langle a, b\rangle_{x}=\frac{1}{2}[(a, x)+(x, b)-(a, b)]$. Pour $\xi$ et $\eta$ distincts dans $\bar{X}$, on peut définir $\langle\xi, \eta\rangle_{x}$ comme la limite de $\langle a, b\rangle_{x}$ quand $X \ni a \rightarrow \xi$ et $X \ni b \rightarrow \eta$. En fait, $\langle\xi, \eta\rangle_{x}=$ $\frac{1}{2}\left[\beta_{\xi}(x, p)+\beta_{\eta}(x, p)\right]$ pour tout $p \in(\xi \eta)$. On définit alors une distance visuelle $d_{x}$ sur $\partial X$, vu du point $x \in X$, par $d_{x}(\xi, \eta)=e^{-\langle\xi, \eta\rangle_{x}}$ pour $\xi$ et $\eta$ distincts dans $\partial X$, et $d_{x}(\xi, \xi)=0$. On peut montrer que $d_{x}$ est bien une distance - ce qui n'est pas évident - induisant la topologie naturelle de $\partial X$, et que la famille de distances $\left\{d_{x}\right\}_{x \in X}$ est primo conforme et secundo invariante par isométrie, c'est-à-dire :

$$
\begin{aligned}
d_{x^{\prime}}(\xi, \eta) & =e^{\frac{1}{2}\left[\beta_{\xi}\left(x, x^{\prime}\right)+\beta_{\eta}\left(x, x^{\prime}\right)\right]} d_{x}(\xi, \eta) \\
d_{\phi x}(\phi \xi, \phi \eta) & =d_{x}(\xi, \eta) .
\end{aligned}
$$

Notons aussi que le $d_{x}$-diamètre de $\partial X$ vaut l'unité (on a d'ailleurs $\langle\xi, \eta\rangle_{x}=0$ si et seulement si $x \in(\xi \eta))$. C'est une conséquence tant utile qu'immédiate de l'inégalité triangulaire que $\langle\xi, \eta\rangle_{x} \leqslant r$ et par suite $d_{x}(\xi, \eta) \geqslant e^{-r}$, où $r$ est la distance de $x$ à la géodésique $(\xi \eta)$. À ce propos, on peut voir que $r=(x, p)$ pour un unique $p \in(\xi \eta)$ que l'on appelle parfois le «projeté orthogonal» de $x$ sur $(\xi \eta)$.

Un dernier point de notations concernant $X$ : on désignera par $B(x, r)$ la boule ouverte dans $X$ de centre $x \in X$ et de rayon $r$, et par $B_{x}(\xi, r)$ la boule ouverte visuelle dans $\partial X$ de centre $\xi$ et de $d_{x}$-rayon $r$.

À l'instar de celles de l'espace hyperbolique, les isométries de $X$ se répartissent en trois types disjoints, l'identité $i d$ mise à part. Une isométrie elliptique fixe au moins un point dans $X$. Une isométrie parabolique fixe un unique point de $\bar{X}$ situé à l'infini ; elle 
préserve toute horosphère basée en ce point. Enfin, une isométrie hyperbolique $\gamma$ fixe exactement deux points dans $\bar{X}$, situés à l'infini ; la géodésique reliant ces deux points fixes est appelée son axe, sur lequel $\gamma$ agit comme une translation dont le déplacement $\ell>0$ est dit longueur de translation de $\gamma$; l'un des points fixes, disons $a$, est attractif, l'autre, $b$, répulsif; on a alors $\beta_{a}(x, \gamma x)=\ell=-\beta_{b}(x, \gamma x)$ pour tout $x \in X$; il en découle que $(x, \gamma x)$ a pour minimum $\ell$, lequel est atteint précisément sur l'axe de $\gamma$.

Le groupe des isométries de $X$ sera muni de sa topologie naturelle. Tout au long du présent article, nous considérerons un groupe d'isométries discret $\Gamma$. Il est de tels groupes discrets qui laissent invariant un ensemble fini de points de $\bar{X}$; on les dit élémentaires, et, hormis le groupe trivial, ils se classent en trois types disjoints. Un groupe discret d'isométries de type elliptique possède au moins un point fixe global dans $X$; il est d'ordre fini et ses éléments non triviaux sont elliptiques. Un groupe de type parabolique possède exactement un point fixe global situé à l'infini; ses éléments non triviaux sont elliptiques (d'ordre fini) ou paraboliques; un tel groupe préserve les horosphères basées en son point fixe. Un groupe de type hyperbolique laisse invariant un unique doublet de points de $\bar{X}$, situés à l'infini; un tel groupe contient un sousgroupe distingué d'indice fini, engendré cycliquement par une isométrie hyperbolique ; les autres éléments non triviaux sont elliptiques. Nous supposerons dans tout ce qui suit que $\Gamma$ n'est pas élémentaire. Il contient alors une infinité de classes de conjugaison d'isométries hyperboliques primitives, c'est-à-dire qui ne sont pas des puissances non triviales d'éléments de $\Gamma$.

L'ensemble limite $\Lambda(\Gamma)$ de $\Gamma$ est par définition l'ensemble des points d'accumulation dans $\bar{X}$ d'une (de toute) orbite de $\Gamma$ dans $X$. Comme $\Gamma$ est discret, on a $\Lambda(\Gamma) \subset \partial X$. Dire que $\Gamma$ n'est pas élémentaire revient à dire que $\Lambda(\Gamma)$ est infini; alors aucun point de $\Lambda(\Gamma)$ n'est isolé, et $\Lambda(\Gamma)$ est le plus petit fermé dans $\bar{X}$ non vide et invariant par $\Gamma$. De plus, $\Gamma$ agit proprement discontinûment sur $\bar{X} \backslash \Lambda(\Gamma)$. Les stabilisateurs des points fixes de $\Gamma$ dans $\bar{X}$, nécessairement situés dans $X \cup \Lambda(\Gamma)$, sont par nature élémentaires. On attribuera à un tel point fixe le type (elliptique, parabolique ou hyperbolique) de son stabilisateur. Les sous-groupes elliptiques ou paraboliques maximaux de $\Gamma$ sont des stabilisateurs de points fixes de $\Gamma$.

\section{B. Densités conformes et invariantes à l'infini}

C'est l'ensemble limite de $\Gamma$ qui est le théâtre de la dynamique de $\Gamma$ dans $\bar{X}$. Aussi a-t-on cherché à munir cet ensemble de mesures en relation avec l'action de $\Gamma$. Du fait que $\Gamma$ n'est pas élémentaire, on voit aisément qu'il ne saurait exister de mesures finies sur $\partial X$, non triviales et invariantes par $\Gamma$. Ce sont toutefois de remarquables mesures quasi invariantes que l'on y a inventées, dans [Patt1], et qui font l'objet de ce paragraphe. Plutôt que de parler de mesures sur le bord à l'infini $\partial X$, il parait ici plus approprié d'adopter le concept de densité, à l'instar de [Sull1]. On appellera densité 
(à l'infini) une application $\mu$ qui à chaque $x \in X$ associe une mesure $\mu_{x}$ positive finie sur $\partial X$.

Une densité $\mu$ sera dite conforme de dimension $\delta \geqslant 0$ si pour tous $x$ et $x^{\prime}$ dans $X$, la mesure $\mu_{x^{\prime}}$ est absolument continue par rapport à $\mu_{x}$, avec une dérivée de RadonNikodym donnée par la formule :

$$
\frac{d \mu_{x^{\prime}}}{d \mu_{x}}(\xi)=e^{-\delta \beta_{\xi}\left(x^{\prime}, x\right)}
$$

Une densité $\mu$ sera dite invariante par $\Gamma$ si pour tout $\gamma \in \Gamma$ et pour tout $x \in X$, on $\mathrm{a}^{(1)}$ :

$$
\gamma_{*} \mu_{x}=\mu_{\gamma x}
$$

Si $\mu$ est une densité conforme et invariante par $\Gamma$, alors la mesure $\mu_{x}(x \in X)$ est quasi invariante par $\Gamma$, c'est-à-dire que $\gamma_{*} \mu_{x}$ est absolument continue par rapport à $\mu_{x}$; mais avec le concept de densité, l'on recouvre une invariance à proprement parler. Non seulement nous ne prendrons en compte que des densités conformes non nulles, mais encore nous les supposerons dorénavant normalisées, en imposant que $\mu_{o}$ soit une probabilité (c'est-à-dire $\left\|\mu_{o}\right\|=1$ ), où o est un point (origine) de $X$ définitivement fixé — c'est ainsi que nous pourrons parler d'unicité dans certaines situations. En outre, nous choisissons o parmi les points non elliptiques, c'est-à-dire de stabilisateur trivial dans $\Gamma$. Remarquons que le support d'une densité conforme et invariante par $\Gamma$ contient nécessairement $\Lambda(\Gamma)$. Nous n'exigerons cependant pas que le support d'une telle densité soit exactement $\Lambda(\Gamma)$.

L'exposant critique $\delta(\Gamma)$ de $\Gamma$ est un nombre fondamental que l'on définit comme étant l'exposant critique de la série de type Dirichlet $\sum_{\gamma \in \Gamma} e^{-s(x, \gamma x)}(s \in \mathbb{R})$, appelée série de Poincaré de $\Gamma$, ce qui revient à dire que

$$
\delta(\Gamma)=\limsup _{t \rightarrow+\infty} \#\{\gamma \in \Gamma \mid(x, \gamma y) \leqslant t\}
$$

(l'exposant ne dépend point du choix de $x$ et $y$ dans $X$ ); la fonction de $t$ précédente est généralement baptisée fonction orbitale de $\Gamma$ - nous en reparlerons notamment dans le chapitre 4 . Nous ferons désormais l'hypothèse que $\delta(\Gamma)$ est fini, hypothèse que la généralité de notre cadre géométrique nous contraint de stipuler, mais qui est d'emblée acquise notamment lorsque $X$ est une variété à courbure pincée (grâce au théorème de comparaison de Rauch, avec un argument volumique), ou encore lorsque $X$ admet un groupe d'isométries discret cocompact.

Les bases de la théorie des densités conformes ont été jetées par Patterson ([Patt1], [Patt3]), à qui l'on doit une construction célèbre, dont nous ne reproduirons pas les détails, et dont nous abstrayons l'énoncé d'existence ci-dessous. La construction en

$\overline{{ }^{(1)} \gamma_{*} \mu_{x} \text { désigne }}$ la mesure définie par $\gamma_{*} \mu_{x}(B)=\mu_{x}\left(\gamma^{-1} B\right)$ pour tout borélien $B$. 
question consiste pour l'essentiel à extraire une limite faible d'une somme convenablement normalisée de masses de Dirac le long d'une orbite de $\Gamma$, pondérées par les termes de la série de Poincaré en $s$, éventuellement quelque peu modifiés, quand $s$ décroît vers $\delta(\Gamma)$. Ce procédé ne requiert que la continuité de la fonction de Busemann (cf. 1A), et se transpose tel quel dans les espaces CAT $(-1)$.

ThÉORÈme 1.1 (Patterson). - Il existe une densité conforme de dimension $\delta(\Gamma)$, invariante par $\Gamma$ et de support $\Lambda(\Gamma)$.

Comme nous savons (voir 1A) ne pas exister de mesure non triviale invariante par $\Gamma$ à l'infini, ou encore de densité conforme de dimension zéro et invariante, il vient directement du théorème 1.1 un fait loin d'être évident : c'est que $\delta(\Gamma)>0$.

À partir de ce théorème d'existence, la théorie a pris un essor remarquable dans les travaux de Sullivan ([Sull1], [Sull2]), dont la pierre angulaire est le lemme 1.3 ci-dessous, connu sous le nom de lemme de l'ombre. Concurremment avec les boules visuelles, nous ferons en effet un grand usage de ce qu'il est convenu d'appeler l'ombre (à l'infini) d'une boule $B(y, r)$ dans $X$ vue d'un point $x \in X$, à savoir :

$$
\left.\left.\mathcal{O}_{r}(x, y)=\{\xi \in \partial X \mid] x \xi\right) \cap B(y, r) \neq \varnothing\right\} .
$$

Enregistrons d'abord un lemme découlant simplement de l'inégalité triangulaire, et dont nous ferons mention à plusieurs reprises.

Lemme 1.2. - Pour tout $\xi \in \mathcal{O}_{r}(x, y)$, on $a(x, y)-2 r<\beta_{\xi}(x, y) \leqslant(x, y)$.

Voici à présent le lemme de l'ombre, conçu à l'origine en courbure constante dans [Sull1].

Lemme 1.3 (Sullivan). - Soit $\mu$ une densité conforme de dimension $\delta$ et invariante par $\Gamma$. Soit $x \in X$. Alors, dès que $r>0$ est assez grand, il existe $C>0$ tel que pour tout $\gamma \in \Gamma$,

$$
\frac{1}{C} e^{-\delta(x, \gamma x)} \leqslant \mu_{x}\left(\mathcal{O}_{r}(x, \gamma x)\right) \leqslant C e^{-\delta(x, \gamma x)}
$$

Démonstration. - En usant des seules définitions, on a

$$
\begin{aligned}
\mu_{x}\left(\mathcal{O}_{r}(x, \gamma x)\right) & =\mu_{x}\left(\gamma \mathcal{O}_{r}\left(\gamma^{-1} x, x\right)\right) \\
& =\mu_{\gamma^{-1} x}\left(\mathcal{O}_{r}\left(\gamma^{-1} x, x\right)\right)=\int_{\mathcal{O}_{r}\left(\gamma^{-1} x, x\right)} e^{-\delta \beta_{\xi}\left(\gamma^{-1} x, x\right)} d \mu_{x}(\xi) .
\end{aligned}
$$

Avec le lemme 1.2, il en résulte que

$$
e^{-\delta(\gamma x, x)} \mu_{x}\left(\mathcal{O}_{r}\left(\gamma^{-1} x, x\right)\right) \leqslant \mu_{x}\left(\mathcal{O}_{r}(x, \gamma x)\right) \leqslant\left\|\mu_{x}\right\| e^{2 \delta r} e^{-\delta(\gamma x, x)} .
$$

Or la borne supérieure des $d_{x}$-diamètres des $\partial X \backslash \mathcal{O}_{r}(y, x), y$ parcourant $X$, tend vers 0 quand $r$ tend vers l'infini. Comme $\mu_{x}$ n'est pas réduite à un atome (parce que 
$\Gamma$ n'est pas élémentaire), on en déduit que

$$
\mu_{x}\left(\mathcal{O}_{r}\left(\gamma^{-1} x, x\right)\right) \geqslant \frac{1}{2}\left[\left\|\mu_{x}\right\|-\max _{\xi \in \partial X} \mu_{x}(\{\xi\})\right]>0
$$

pourvu que $r$ soit assez grand (indépendamment de $\gamma$ ), ce qui achève la démonstration.

Une première conséquence importante de ce résultat, décrite dans [Sull1], est que la dimension $\delta$ de $\mu$ est nécessairement supérieure ou égale à $\delta(\Gamma)$. On prouve simultanément que la fonction orbitale de $\Gamma$ ( $c f$. supra) est majorée par une constante fois $e^{\delta(\Gamma) t}$, estimation que l'on verra largement précisée par le théorème 4.1 plus loin. Ce double corollaire repose sur un argument que l'on verra réapparaître dans la preuve du théorème 5.2 plus loin; il consiste surtout à observer que le recouvrement de $\partial X$ par les ombres $\mathcal{O}_{r}(x, \gamma x)$, où $t-1<(x, \gamma x) \leqslant t$, est de multiplicité comprise entre zéro et une constante indépendante de $t$, le lemme de l'ombre faisant le reste.

Plutôt que d'étudier abruptement l'action de $\Gamma$ à l'infini, c'est-à-dire un système dynamique $\left(\partial X, \Gamma, \mu_{o}\right)$ où $\mu$ est une densité conforme invariante, on a préféré se tourner vers des extensions de ce dernier, à commencer par le flot géodésique.

\section{C. Flot géodésique}

Notons $S X$ l'ensemble des isométries de $\mathbb{R}$ dans $X$, que l'on munira de sa topologie naturelle. Autrement dit, $S X$ est l'espace des géodésiques de $X$ paramétrées par $\mathbb{R}$. Dans le cas où $X$ est une variété, $S X$ s'identifie au fibré unitaire tangent à $X$, d'où la notation employée. On dispose d'une «projection» canonique de $S X$ sur $X$, qui à un point de $S X$ associe ce qu'on appellera son point de base. Signalons que dans notre cadre général, cette projection peut ne pas être surjective, les géodésiques n'étant pas toutes bi-infinies; ce défaut n'aura toutefois aucune incidence, comme nous l'avons déjà annoncé. Nous commettrons fréquemment par la suite l'abus de notation consistant à confondre un point de $S X$ et son point de base dans des expressions où cela ne pourra être source d'ambiguité; c'est ainsi que nous écrirons $\beta_{\xi}(x, u)$ avec $u \in S X$, etc. Pour $B \subset X$, nous noterons $S B$ l'image réciproque de $B$ par la projection de $S X$ dans $X$. Enfin, l'on voit facilement que le groupe des isométries de $X$ agit librement par homéomorphismes sur $S X$, et que $\Gamma$ agit proprement, discontinûment et librement sur $S X$.

Le flot trivial sur $\mathbb{R}$ induit un flot continu $\left\{g^{t}\right\}_{t \in \mathbb{R}}$ agissant sur $S X$, appelé flot géodésique. De plus, chaque $g^{t}$ commute avec les isométries de $X$ agissant sur $S X$. Pour $u \in S X$, nous noterons $g^{+\infty} u$ (resp. $g^{-\infty} u$ ) l'extrémité à l'infini suivant les temps positifs (resp. négatifs) de la géodésique déterminée par $u$. Autrement dit, $g^{+\infty} u$ (resp. $g^{-\infty} u$ ) est la limite (dans $\bar{X}$ ) du point de base de $g^{t} u$ quand $t$ tend vers $+\infty$ $($ resp. $-\infty)$. 
La symétrie de centre 0 dans $\mathbb{R}$ induit pour sa part une involution $\mathcal{I}$ de $S X$, qui agit par renversement de l'orientation : $g^{ \pm \infty}=g^{\mp \infty} \circ \mathcal{I}$, et par « renversement du temps » sur le flot géodésique $: \mathcal{I} \circ g^{t}=g^{-t} \circ \mathcal{I}$.

Nous adoptons la notation usuelle $\partial^{2} X$ pour le produit de $\partial X$ par lui-même privé de sa diagonale. Dans tout ce qui suit, nous identifierons $S X \grave{a} \partial^{2} X \times \mathbb{R}$ par l'homéomorphisme qui à $u \in S X$ associe $\left(g^{-\infty} u, g^{+\infty} u, \beta_{g^{-\infty}} u(u, o)\right)$. Seule la projection de $S X$ sur $\partial^{2} X$ est canonique, la composante réelle dans l'identification précédente étant définie seulement à translation près. En décrivant $u \in S X$ par ses coordonnées $(\xi, \eta, s) \in \partial^{2} X \times \mathbb{R}$, nous avons $g^{t} u=(\xi, \eta, s+t)$ et $\phi u=\left(\phi \xi, \phi \eta, s+\beta_{\xi}\left(o, \phi^{-1} o\right)\right)$. Notons enfin que $S X$ est métrisable.

Introduisons maintenant des mesures de Radon sur $S X$ invariantes à la fois par le flot géodésique et par $\Gamma$, formées à partir des densités conformes invariantes par $\Gamma$ du paragraphe précédent, selon une construction employée dans [Sull1]. Il se trouve que les mesures ainsi obtenues coïncident dans certains cadres avec celles étudiées par de nombreux auteurs suivant des voies différentes. Aussi avons-nous choisi ici de les appeler mesures de Bowen-Margulis-Sullivan (BMS en abrégé). Étant donnée une densité $\mu$ conforme de dimension $\delta$ et invariante par $\Gamma$, nous lui associons la mesure $m$ de BMS sur $S X$ définie par

$$
d m(u)=\frac{d \mu_{x}(\xi) d \mu_{x}(\eta) d s}{d_{x}(\xi, \eta)^{2 \delta}} \quad \text { avec } u=(\xi, \eta, s) \in S X \text {, où } x \in X \text { est fixé. }
$$

On constate sans peine que la mesure $m$ ne dépend point du choix de l'identification de $S X$ à $\partial^{2} X \times \mathbb{R}$; elle est donc intrinsèque. Grâce à la conformité de $\mu$, la mesure $m$ ne dépend pas davantage du choix de $x \in X$; notons d'ailleurs la formule suivante :

$$
d m(u)=e^{\delta \beta_{\xi}(x, u)+\delta \beta_{\eta}(x, u)} d \mu_{x}(\xi) d \mu_{x}(\eta) d s .
$$

L'invariance par $\Gamma$ de $m$ découle alors de celle de $\mu$. Quant à l'invariance de $m$ par le flot géodésique dans $S X$, ainsi que par $\mathcal{I}$, elle est triviale. Lorsque $X$ est un espace hyperbolique, la mesure de BMS associée à la densité de Lebesgue est mieux connue sous le nom de mesure de Liouville.

Nous considérerons épisodiquement des mesures quelque peu plus générales : si $\nu$ et $\mu$ sont deux densités conformes de dimensions respectives $\delta_{\nu}$ et $\delta_{\mu}$, et invariantes par $\Gamma$, nous leur associerons la mesure de BMS généralisée $m^{\nu, \mu}$ donnée par

$$
d m^{\nu, \mu}(u)=e^{\delta_{\nu} \beta_{\xi}(x, u)+\delta_{\mu} \beta_{\eta}(y, u)} d \nu_{x}(\xi) d \mu_{y}(\eta) d s
$$

avec $u=(\xi, \eta, s) \in S X$, où $x$ et $y$ sont fixés dans $X$. Là encore, on vérifie rapidement que $m^{\nu, \mu}$ ne dépend point du choix de $x$ ni de $y$, que $m^{\nu, \mu}$ est invariante par $\Gamma$, et enfin que $\left(g^{t}\right)_{*} m^{\nu, \mu}=e^{\left(\delta_{\nu}-\delta_{\mu}\right) t} m^{\nu, \mu}(t \in \mathbb{R})$ et $\mathcal{I}_{*} m^{\nu, \mu}=m^{\mu, \nu}$. La mesure $m$ que nous définissions plus haut n'était autre que $m^{\mu, \mu}$.

Les quotients de $g^{t}$ et de $m$ par $\Gamma$ seront notés respectivement $g_{\Gamma}^{t}$ et $m_{\Gamma}$. Nous étudierons par la suite les systèmes dynamiques $\left(S X / \Gamma,\left\{g_{\Gamma}^{t}\right\}_{t \in \mathbb{R}}, m_{\Gamma}\right)$. Lorsqu'à l'avenir 
nous parlerons de flot géodésique et de mesures de BMS, nous entendrons généralement par là leurs quotients suivant $\Gamma$. Cependant, les démonstrations se dérouleront d'ordinaire dans le « revêtement universel » $S X$. Nous aurons à ce moment-là besoin de domaines fondamentaux appropriés pour l'action de $\Gamma$, au sujet desquels il convient de dire quelques mots.

On appellera domaine fondamental pour l'action de $\Gamma$ sur l'un des espaces $\bar{X} \backslash \Lambda(\Gamma)$ ou $S X$, tout ouvert $D$ tel que $\gamma D \cap D=\varnothing$ pour tout $\gamma \in \Gamma \backslash\{\operatorname{Id}\}$, et tel que la réunion $\Gamma \bar{D}$ des images de $D$ par $\Gamma$ couvre l'espace considéré. Un tel domaine est dit localement fini si tout compact donné ne rencontre qu'un nombre fini d'images de $\bar{D}$. Il est facile de s'assurer de l'existence d'un domaine fondamental localement fini pour l'action de $\Gamma$ sur $\bar{X} \backslash \Lambda(\Gamma)$, et partant sur $X$ puis $S X$, en reprenant la construction classique de Dirichlet : si $a \in X$ est un point qui n'est fixé par aucun élément non trivial de $\Gamma$ (par exemple $a=o$ ), alors l'ensemble des points $x \in X$ tels que $(x, a)<(x, \gamma a)$ pour tout $\gamma \in \Gamma \backslash\{\operatorname{Id}\}$ est un domaine possédant les propriétés susindiquées, du seul fait que $\Gamma$ est discret; il est appelé domaine de Dirichlet de centre a pour $\Gamma$. Dans $S X$ ou $\bar{X} \backslash \Lambda(\Gamma)$, les domaines fondamentaux convenables seront ceux dont le bord est négligeable pour une mesure quasi invariante par $\Gamma$ donnée, pourvu qu'elle ne donne aucune masse à l'ensemble des points fixes de $\Gamma$, ce qui sera bien notre situation en temps utile (voir corollaire 1.8). Il en existe effectivement, puisque l'on peut modifier comme suit le bord d'un domaine fondamental localement fini $D_{0}$ de façon à obtenir la propriété voulue. En notant $F$ l'ensemble des points fixes, on peut en effet recouvrir $\partial D_{0} \backslash F$ par une famille localement finie dans $F^{c}$ d'ouverts $V_{1}, V_{2}, \ldots$ dont le bord est négligeable pour la mesure quasi invariante donnée et dont les images par $\Gamma$ sont d'adhérences disjointes; comme $D_{0}$ est localement fini, la famille $\Gamma V_{1}, \Gamma V_{2}, \ldots$ est encore localement finie dans $F^{c}$; en définissant par récurrence $D_{n}=\left(D_{n-1} \backslash \Gamma \overline{V_{n}}\right) \cup V_{n}(n \geqslant 1)$, on obtient par conséquent une suite de domaines fondamentaux dans $F^{c}$ localement stationnaire; sa limite $D$ est encore un domaine fondamental localement fini, mais cette fois de bord négligeable car contenu dans la réunion de $F$ et des $\Gamma \partial V_{n}$. Rappelons enfin l'usage d'un tel domaine $D$ : pour toute fonction $h$ appartenant à l'espace $C_{c}(S X / \Gamma)$ des fonctions continues à support compact sur $S X / \Gamma$, que l'on relève en une fonction $\widetilde{h}$ sur $S X$, on vérifie aisément que la quantité $\int_{D} \widetilde{h} d m$ ne dépend pas de $D$, et qu'en fait $\int_{D} \widetilde{h} d m=\int_{S X / \Gamma} h d m_{\Gamma}$.

Effleurons pour finir la question des géodésiques fermées sur le quotient $S X / \Gamma$ : en l'absence de torsion dans $\Gamma$, ce sont précisément les orbites périodiques du flot géodésique, mais aussi les projetés dans $S X / \Gamma$ des axes des isométries hyperboliques de $\Gamma$ orientés — nous voulons dire par là relevés naturellement dans $S X$ où on les parcourra du point fixe répulsif vers l'attractif. Dans notre généralité, où il est permis que $\Gamma$ contienne des éléments elliptiques, ces derniers ne fournissent plus toujours de véritables géodésiques fermées, mais plutôt des chemins à extrémités qui se recollent éventuellement moyennant un élément de torsion, comme cela a été formalisé dans 
[G-H] (chapitre 11). Cependant, dans le souci de ne pas alourdir inutilement l'exposition, nous persisterons à appeler géodésiques fermées les projetés dans $S X / \Gamma$ des axes orientés des isométries hyperboliques de $\Gamma$. L'ensemble des longueurs des géodésiques fermées relatives à $\Gamma$, communément dénommé spectre des longueurs de $\Gamma$, jouera un rôle dans tout ce qui suit.

\section{D. Feuilletage horosphérique}

L'étude des propriétés dynamiques du flot géodésique a depuis longtemps mis au tout premier plan les ensembles particuliers que sont les variétés stable et instable. Pour $u \in S X$, la «variété » stable (resp. instable) de $u$, que nous noterons $H^{-}(u)$ (resp. $H^{+}(u)$ ), est par définition l'ensemble des $v \in S X$ tels que la distance de $g^{t} v$ à $g^{t} u$ tend vers 0 quand $t \rightarrow+\infty$ (resp. $\left.-\infty\right)$. Il est clair que $H^{-}(u)$ (resp. $H^{+}(u)$ ) est l'ensemble des $v \in S X$ tels que $g^{+\infty} v=g^{+\infty} u$ (resp. $g^{-\infty} v=g^{-\infty} u$ ) et que $v$ soit basé sur l'horosphère basée en $g^{+\infty} u$ (resp. $g^{-\infty} u$ ) et passant par le point de base de $u$. Aussi $H^{-}(u)$ (resp. $H^{+}(u)$ ) sera-t-elle désormais appelée, en toute légitimité, l'horosphère stable (resp. instable) de u.

L'ensemble des horosphères stables (resp. instables) pour le flot géodésique dans $S X$ constitue une partition de $S X$, que l'on appellera le "feuilletage horosphérique stable (resp. instable)», qui constitue effectivement un feuilletage au sens usuel lorsque $X$ est une variété. Ces deux feuilletages se déduisent l'un de l'autre par renversement de l'orientation, vu que $H^{+}(u)=\mathcal{I} H^{-}(\mathcal{I} u)$. Aussi ferons-nous abstraction des directions sur les horosphères (in)stables pour ne plus considérer que l'espace $\mathcal{H}$ des horosphères de $X$ : c'est cet objet que nous appellerons le « feuilletage horosphérique» tout court. Le groupe des isométries de $X$ agit naturellement sur $\mathcal{H}$, corrélativement à son action sur les feuilletages stable et instable.

De même que nous avions identifié $S X$ à $\partial^{2} X \times \mathbb{R}$ (voir $1 \mathrm{C}$ ), de même allons-nous assimiler $\mathcal{H}$ à $\partial X \times \mathbb{R}$ par l'homéomorphisme qui à une horosphère $H \in \mathcal{H}$ de base $\xi \in \partial X$ et passant par un point $h \in X$ associe $\left(\xi, \beta_{\xi}(h, o)\right)$ (ce qui ne dépend pas du choix de $h$ ). Seule la projection sur $\partial X$ est canonique, la composante réelle n'étant bien définie qu'à translation près. En décrivant $H \in \mathcal{H}$ par ses coordonnées $(\xi, s)$, nous avons $\phi(\xi, s)=\left(\phi \xi, s+\beta_{\xi}\left(o, \phi^{-1} o\right)\right)$ pour toute isométrie $\phi$.

Nous allons faire de l'action de $\Gamma$ sur $\mathcal{H}$ un système dynamique qui, par son origine même, manifestera des liens étroits avec le flot géodésique, lesquels se révéleront fructueux par la suite - ceci constitue le principe même des chapitres 2,3 et 4 . Il convient avant tout de discuter la notion de mesure invariante pour l'action de $\Gamma$ sur $\mathcal{H}$, certaines situations particulières offrant des points de vue multiples. Il est tout d'abord un cas simple où la notion de mesure invariante est ordinaire : en effet, lorsque $\partial X$ est homéomorphe au cercle (notamment lorsque $X$ est une surface), on peut orienter 
les horosphères - alors appelées horocycles - (in)stables, puis les voir comme les orbites d'un flot dans $S X$ dit horocyclique (in)stable; on s'intéressera alors aux mesures invariantes par le flot horocyclique (in)stable et par $\Gamma$; dans le cas où $X$ est le disque hyperbolique, on peut donner à titre d'exemple les mesures du type $m^{\mu, \lambda}$ où $\mu$ est une densité conforme invariante par $\Gamma$ et $\lambda$ la densité de Lebesgue. Conditionnée sur une orbite du flot horocyclique, une telle mesure invariante se réduit à celle de Lebesgue; en effectuant une désintégration, on se convainc alors de la compatibilité de cette notion avec la suivante, valable cette fois dans le cadre plus général d'un (véritable) feuilletage. On se représente une mesure invariante par un feuilletage comme une famille de mesures sur les variétés transversales à ce dernier, invariante par holonomie. Pour un feuilletage topologiquement transitif, ce qui sera bientôt notre situation, une telle famille est en fait déterminée par un seul de ses éléments. Dans le cas où $X$ est une variété, on peut choisir pour transversale au feuilletage horosphérique stable le produit d'une géodésique et d'une horosphère instable se rencontrant, ce que l'on ramène, par projection de cette dernière à l'infini suivant les géodésiques, à $\partial X \times \mathbb{R}=\mathcal{H}$. Les isométries commutant avec l'holonomie du feuilletage en question, on voit de cette façon que les mesures invariantes à la fois par le feuilletage horosphérique stable et par $\Gamma$ correspondent aux mesures sur $\mathcal{H}$ invariantes par $\Gamma$. Il nous est donc tout à fait loisible d'étendre dans notre cadre général la notion de mesure invariante à la fois par le feuilletage horosphérique (in)stable et par $\Gamma$, tout simplement en appelant telle une mesure sur $\mathcal{H}$ invariante par $\Gamma$. Si $\nu$ est une telle mesure, nous étudierons le système dynamique $(\mathcal{H}, \Gamma, \nu)$.

Une classe de mesures sur $\mathcal{H}$ invariantes par $\Gamma$ s'impose, que l'on tire des densités conformes à la façon des mesures de BMS. À chaque densité $\mu$ conforme de dimension $\delta$ et invariante par $\Gamma$, nous associerons la mesure $\widehat{\mu}$ sur $\mathcal{H}$, invariante par $\Gamma$, donnée par :

$$
d \widehat{\mu}(H)=d \mu_{x}(\xi) e^{\delta \beta_{\xi}(x, h)} d s
$$

avec $H=(\xi, s) \in \mathcal{H}$, et $h$ un point quelconque de $H$ dans $X$. Il est aisé de vérifier l'indépendance de $\widehat{\mu}$ par rapport au point $x$ fixé dans $X$, puis son invariance par $\Gamma$. On constate qu'en outre $\widehat{\mu}$ ne dépend point du choix de l'identification de $\mathcal{H}$ à $\partial X \times \mathbb{R}$, et partant qu'elle est intrinsèque. Signalons enfin qu'avec $x=o$, la formule ci-dessus devient

$$
d \widehat{\mu}(H)=d \mu_{o}(\xi) e^{-\delta s} d s .
$$

Refermons cette présentation du feuilletage horosphérique sur des rappels concernant ses caractéristiques topologiques, qui feront d'ores et déjà ressortir son attache avec le flot géodésique (voir 1C pour ce qui a trait à ce dernier). Il revient à F. Dal'bo ([Dal]) d'avoir achevé d'éclaircir les relations entre les assertions de l'énoncé cidessous. Détailler les preuves des deux résultats suivants, qui au surplus ne nous seront utiles qu'une seule fois (théorème 6.4), nous eût conduit à par trop paraphraser [Dal]. Quoique le contexte adopté dans ce dernier travail fût celui des variétés riemanniennes 
à courbure pincée, tant les énoncés que les démonstrations s'adaptent directement au cadre élargi des espaces CAT $(-1)$ (en particulier, le lemme de fermeture qui y est utilisé reste valable).

THÉORÈME 1.4 (Dal'bo). — Les trois assertions suivantes sont équivalentes :

(i) Le spectre des longueurs associé à $\Gamma$ n'est pas arithmétique, c'est-à-dire qu'il engendre un sous-groupe dense de $\mathbb{R}$.

(ii) Le feuilletage horosphérique restreint à $\Lambda(\Gamma) \times \mathbb{R} \subset \mathcal{H}$ est topologiquement transitif, c'est-à-dire qu'il existe une horosphère dont l'orbite suivant $\Gamma$ est dense dans $\Lambda(\Gamma) \times \mathbb{R}$.

(iii) Le flot géodésique $\left\{g_{\Gamma}^{t}\right\}$ restreint au quotient par $\Gamma$ de $S X \cap(\Lambda(\Gamma) \times \Lambda(\Gamma) \times \mathbb{R})$ est topologiquement mélangeant, c'est-à-dire que pour tous ouverts $U$ et $V$ de $S X / \Gamma$ rencontrant l'ensemble précédent, on a $U \cap g_{\Gamma}^{-t} V \neq \varnothing$ dès que $|t|$ est assez grand.

On définit l'ensemble limite horosphérique $\Lambda_{h}(\Gamma)$ de $\Gamma$ comme l'ensemble des points $\xi$ de $\partial X$ (dits limites horosphériques) tels que toute horoboule basée en $\xi$ rencontre l'orbite d'un (de tout) point de $X$ fixé. Évidemment, $\Lambda_{h}(\Gamma) \subset \Lambda(\Gamma)$. La proposition suivante confirme l'importance de cet ensemble.

Proposition 1.5 (Hedlund, Dal'bo). - Supposons que les conditions équivalentes de la proposition précédente soient satisfaites. Alors l'orbite suivant $\Gamma$ d'une horosphère de $\mathcal{H}$ est dense dans $\Lambda(\Gamma) \times \mathbb{R} \subset \mathcal{H}$ si et seulement si sa base est dans $\Lambda_{h}(\Gamma)$.

Dès lors, il va sans dire que la question de la non-arithméticité du spectre des longueurs associé à $\Gamma$ paraît cruciale dans l'étude du feuilletage horosphérique. De fait, nous nous heurterons encore à elle au sujet de l'ergodicité de ce dernier (chapitre 2), et serons contraints de l'admettre tout au long du restant de l'article (chapitres 3 à 6), qui est fondé sur le corollaire 2.3. Aussi n'est-il peut-être pas inutile de signaler que cette condition est d'avance acquise dans un bon nombre de situations, où l'on peut plus ou moins facilement la vérifier, alors qu'elle a jusqu'à présent résisté aux investigations dans le cas général - on conjecture cependant qu'elle est vraie lorsque $X$ est, par exemple, une variété, tandis qu'elle est clairement invalide lorsqu'à l'opposé, $X$ est un arbre à longueur d'arêtes uniforme. Voici l'état de ce problème ouvert tel qu'on peut le dresser aujourd'hui. Nous renvoyons le lecteur à [Dal] pour les références.

Proposition 1.6. - Le spectre de $\Gamma$ n'est pas arithmétique dans les cas suivants :

(1) $X$ est une surface riemannienne (dimension deux);

(2) $X$ est un espace hyperbolique (courbure constante);

(3) $\Lambda(\Gamma)$ possède une composante connexe non réduite à un point;

(4) $\Gamma$ contient une isométrie parabolique.

Notons que le cas (3) se produit lorsque $\Gamma$ est cocompact et $\partial X$ est connexe. 


\section{E. Ergodicité du flot géodésique et groupes de type divergent}

Cette section est consacrée au critère d'ergodicité du flot géodésique connu sous le nom de théorème de Hopf-Tsuji-Sullivan (nous indiquons quelques références en début de preuve). Si ce résultat est d'ores et déjà assez familier dans son ensemble, les démonstrations proposées jusqu'alors paraissent peu ou prou entachées de lacunes, à tout le moins en ce qui concerne certaines tentatives de généralisation en courbure variable. Aussi avons-nous tenu à établir ici un énoncé d'une part complet dans sa formulation, et d'autre part valable dans les espaces CAT $(-1)$ généraux (théorème 1.7), et à exposer brièvement une démonstration unifiée, au risque de lasser le lecteur par certains arguments rebattus.

Auparavant, il convient de rappeler quelques notions fondamentales de la théorie ergodique. Nous avons déjà introduit au cours des paragraphes précédents plusieurs systèmes dynamiques $(\Omega, G, M)$ où $\Omega$ est un espace topologique séparé, localement compact et $\sigma$-compact, $G$ un groupe qui sera dénombrable $(\Gamma)$ ou $\mathbb{R}$ (le flot géodésique), et $M$ une mesure de Radon, finie ou non, quasi invariante par $G$ (pour tout $g \in G, g_{*} M$ est absolument continue par rapport à $\left.M\right)$. Nous avons là l'archétype des systèmes $\left(\partial X, \Gamma, \mu_{o}\right),\left(S X / \Gamma,\left\{g_{\Gamma}^{t}\right\}_{t \in \mathbb{R}}, m_{\Gamma}\right),(\mathcal{H}, \Gamma, \widehat{\mu}), \ldots$ Notons $d g$ une mesure de Haar sur $G$ (si $G=\Gamma$, ce sera la mesure de dénombrement, et si $G=\mathbb{R}$, il s'agira de la mesure de Lebesgue). Un borélien $E \subset \Omega$ est dit errant si $\int_{G} \mathbb{1}_{E}(g \omega) d g$ est finie pour presque tout $\omega \in E$. Par un classique argument d'extension ([Kre], page 17), l'espace $\Omega$ se décompose en deux parties $\Omega_{C}$ et $\Omega_{D}$, disjointes et invariantes par $G$, où $\Omega_{D}$ est une réunion dénombrable d'ensembles errants, maximale dans le sens que tout ensemble errant est contenu dans $\Omega_{D}$ modulo $M$. On appelle $\Omega_{D}$ la partie dissipative de $(\Omega, G, M)$, et le complémentaire $\Omega_{C}$ sa partie conservative (elles sont définies modulo $M)$. Si $B$ est un borélien contenu dans $\Omega_{C}$, alors $\int_{G} \mathbb{1}_{B} \circ g d g=+\infty$ presque partout sur $B$; notamment, l'orbite de presque tout point de $B$ retourne indéfiniment dans $B$. Le système $(\Omega, G, M)$ sera dit complètement conservatif (resp. dissipatif) si $M\left(\Omega_{D}\right)=0$ (resp. $\left.M\left(\Omega_{C}\right)=0\right)$. D'autre part, le système $(\Omega, G, M)$ sera dit ergodique si tout borélien $A$ invariant par $G$ est de mesure pleine ou nulle. Au lieu d'invariant, on peut entendre ici essentiellement invariant, c'est-à-dire que $M(A \Delta g A)=0$ pour tout $g \in G$, et c'est ce que nous ferons par la suite sauf mention contraire (dans le cas où $G$ est un flot, cette variante requiert sa continuité). On vérifie aisément que si $G$ est dénombrable, si $M$ n'a pas d'atomes et si $(\Omega, G, M)$ est ergodique, alors $(\Omega, G, M)$ est complètement conservatif. C'est là pour ainsi dire le seul lien entre les deux notions que nous venons de rappeler.

Venons-en au théorème de dichotomie du comportement ergodique du flot géodésique. Il mettra en relief l'ensemble limite conique $\Lambda_{c}(\Gamma)$ de $\Gamma$, défini comme l'ensemble des points $\xi$ de $\partial X$ (dits limites coniques) qui sont indéfiniment approchés par des 
points de l'orbite d'un (de tout) point fixé dans $X$ qui restent à distance bornée d'un (de tout) rayon géodésique donné finissant en $\xi$. Il est clair que $\Lambda_{c}(\Gamma) \subset \Lambda_{h}(\Gamma)$.

THÉORÈME 1.7. - Soit $\mu$ une densité conforme de dimension $\delta$ et invariante par $\Gamma$, et soit $m_{\Gamma}$ la mesure de Bowen-Margulis-Sullivan qui lui est associée. Dans chacun des deux cas complémentaires suivants, les assertions (i) à (iv) sont simultanément vraies.

Premier cas :

(i) $\sum_{\gamma \in \Gamma} e^{-\delta(o, \gamma o)}<\infty$.

(ii) $\mu_{o}\left(\Lambda_{c}(\Gamma)\right)=0$.

(iii) $\left(\partial^{2} X, \Gamma, \mu_{o} \otimes \mu_{o \mid \partial^{2} X}\right)$ est complètement dissipatif et non ergodique.

(iv) $\left(S X / \Gamma,\left\{g_{\Gamma}^{t}\right\}_{t \in \mathbb{R}}, m_{\Gamma}\right)$ est complètement dissipatif et non ergodique.

Second cas :

(i) $\sum_{\gamma \in \Gamma} e^{-\delta(o, \gamma o)}=\infty$ (alors $\delta=\delta(\Gamma)$.)

(ii) $\mu_{o}\left(\Lambda_{c}(\Gamma)\right)=1$.

(iii) $\left(\partial X \times \partial X, \Gamma, \mu_{o} \otimes \mu_{o}\right)$ est complètement conservatif et ergodique.

(iv) $\left(S X / \Gamma,\left\{g_{\Gamma}^{t}\right\}_{t \in \mathbb{R}}, m_{\Gamma}\right)$ est complètement conservatif et ergodique.

Si la série de Poincaré de $\Gamma$ diverge en $\delta(\Gamma)$, on dira que $\Gamma$ est de type divergent. Sinon, $\Gamma$ sera dit de type convergent.

Un dernier sous-ensemble remarquable de $\Lambda(\Gamma)$ jouera un rôle de premier plan dans le chapitre 2 plus loin : il s'agit des points de Myrberg, dont l'ensemble sera noté $\Lambda_{M}(\Gamma)$. Un point $\xi \in \partial X$ est dit de Myrberg si toute géodésique orientée à extrémités dans $\Lambda(\Gamma)$ est limite d'une suite d'images par $\Gamma$ d'un (de tout) rayon géodésique orienté finissant en $\xi$, autrement dit si pour tous $a$ et $b$ distincts dans $\Lambda(\Gamma)$, il existe $\left\{\gamma_{n}\right\} \subset \Gamma$ telle que $\gamma_{n} o \rightarrow a$ et $\gamma_{n} \xi \rightarrow b$. On trouve dans [ $\left.\mathbf{T u}\right]$ une étude élémentaire des points de Myrberg. Il est clair que $\Lambda_{M}(\Gamma) \subset \Lambda_{c}(\Gamma)$. À l'inverse, les points fixes hyperboliques sont des points limites coniques qui ne sont pas de Myrberg. La dernière affirmation du corollaire suivant du théorème 1.7 sera employée pour le corollaire 2.3 ; elle généralise le résultat de [Tu] à l'aide d'un bref argument.

Corollaire 1.8. - Si $\Gamma$ est de type divergent, il existe une unique densité $\mu$ conforme de dimension $\delta(\Gamma)$ et invariante par $\Gamma$. Elle n'a pas d'atomes, et l'ensemble des points fixes de $\Gamma$ dans $S X$ est négligeable pour la mesure de Bowen-MargulisSullivan associée.

De plus, on a $\mu_{o}\left(\Lambda_{M}(\Gamma)\right)=1$.

Avant de passer aux démonstrations, introduisons dès à présent une classe de groupes particulièrement importante, que nous ne laisserons de rencontrer tout au long de cet article : il s'agit des groupes admettant une mesure de BMS finie. Si $\Gamma$ est un tel groupe, et $m_{\Gamma}$ la mesure de BMS finie en question, alors $\left(S X / \Gamma,\left\{g_{\Gamma}^{t}\right\}_{t \in \mathbb{R}}, m_{\Gamma}\right)$ est conservatif, d'après le théorème de récurrence de Poincaré $(c f .[\mathbf{K r e}],[\mathbf{W}])$. Par le 
théorème précédent, $\Gamma$ est alors de type divergent; en particulier, $m_{\Gamma}$ est unique, et la dimension de la densité associée est $\delta(\Gamma)$.

Démonstration. - Le théorème 1.7 découle des huit affirmations suivantes, que nous allons tour à tour établir. Nous paraissent nouvelles (c) et, en courbure variable, (g). Pour ce qui est de (f), nous proposons ici une extension de la méthode de $[\mathbf{A}-\mathbf{S}]$ où était traité le cas particulier de la mesure de Liouville en courbure constante. Cela mis à part, le reste des arguments a son origine dans [Sull1] (où avait été établi le cas $\delta=\delta(\Gamma)>d / 2$ dans l'espace hyperbolique de dimension $d$ par une méthode de théorie du potentiel). On pourra entre autres consulter $[\mathbf{T u}],[\mathbf{Y}]$ et $[\mathbf{K a 1}]$ sur ce sujet.

(a) Si $\sum_{\gamma \in \Gamma} e^{-\delta(o, \gamma o)}<\infty$, alors $\mu_{o}\left(\Lambda_{c}(\Gamma)\right)=0$.

(b) Si $\mu_{o}\left(\Lambda_{c}(\Gamma)\right)=0$, alors $\left(\partial^{2} X, \Gamma, \mu_{o} \otimes \mu_{o \mid \partial^{2} X}\right)$ est complètement dissipatif.

(c) $\operatorname{Si}\left(\partial^{2} X, \Gamma, \mu_{o} \otimes \mu_{o \mid \partial^{2} X}\right)$ est ergodique, alors $\mu_{o}$ n'a pas d'atomes - et $(\partial X \times$ $\left.\partial X, \Gamma, \mu_{o} \otimes \mu_{o}\right)$ est ergodique et complètement conservatif.

(d) $\forall u \in S X, g^{+\infty} u \in \Lambda_{c}(\Gamma)$ si et seulement si il existe un compact $K \subset S X$ tel que $\int_{0}^{+\infty} \mathbb{1}_{\Gamma K} \circ g^{t}(u) d t=\infty$. Ainsi $\left(S X / \Gamma,\left\{g_{\Gamma}^{t}\right\}_{t \in \mathbb{R}}, m_{\Gamma}\right)$ est complètement conservatif (resp. dissipatif) si et seulement si $\mu_{o}\left(\Lambda_{c}(\Gamma)\right)=1\left(\right.$ resp. $\left.\mu_{o}\left(\Lambda_{c}(\Gamma)\right)=0\right)$.

(e) $\left(S X / \Gamma,\left\{g_{\Gamma}^{t}\right\}_{t \in \mathbb{R}}, m_{\Gamma}\right)$ est ergodique $\Leftrightarrow\left(\partial^{2} X, \Gamma, \mu_{o} \otimes \mu_{o \mid \partial^{2} X}\right)$ est ergodique.

(f) $\mathrm{Si} \sum_{\gamma \in \Gamma} e^{-\delta(o, \gamma o)}=\infty$, alors $\mu_{o}\left(\Lambda_{c}(\Gamma)\right)>0$.

(g) Si $\mu_{o}\left(\Lambda_{c}(\Gamma)\right)>0$, alors $\mu_{o}\left(\Lambda_{c}(\Gamma)\right)=1$.

(h) Si $\left(S X / \Gamma,\left\{g_{\Gamma}^{t}\right\}_{t \in \mathbb{R}}, m_{\Gamma}\right)$ est complètement conservatif, alors il est ergodique.

Il est aisé de voir comment (d) provient de la définition de $\Lambda_{c}(\Gamma)$; (a) découle de cette dernière conjuguée au lemme de l'ombre 1.3, et (e) est triviale.

Prouvons (b). À chaque $(\xi, \eta) \in \partial^{2} X$ avec $\xi \notin \Lambda_{c}(\Gamma)$ et $\eta \notin \Lambda_{c}(\Gamma)$, associons l'ensemble fini non vide $\Gamma_{\xi, \eta}$ des $\gamma \in \Gamma$ tels que la distance de $\gamma o$ à $(\xi \eta)$ soit minimale. Pour toute partie finie non vide $\mathcal{F}$ de $\Gamma$, soit $E_{\mathcal{F}}$ l'ensemble (borélien) des $(\xi, \eta)$ tels que $\Gamma_{\xi, \eta}=\mathcal{F}$. Si $\gamma E_{\mathcal{F}}$ rencontre $E_{\mathcal{F}}$, alors $\gamma \mathcal{F}=\mathcal{F}$; le stabilisateur de $\mathcal{F}$ dans $\Gamma$ étant fini, $E_{\mathcal{F}}$ est errant. Comme l'ensemble des parties finies non vides de $\Gamma$ est dénombrable, on voit que le carré de $\partial X \backslash \Lambda_{c}(\Gamma)$ privé de la diagonale est contenu dans la partie dissipative. On en déduit (b).

Prouvons (c). Supposons par l'absurde que $\mu_{o}$ possède un atome $\xi \in \partial X$, en même temps que $\left(\partial^{2} X, \Gamma, \mu_{o} \otimes \mu_{o \mid \partial^{2} X}\right)$ est ergodique. Prenons $\phi \in \Gamma$ tel que $\phi \xi \neq \xi$, et considérons l'orbite $\mathcal{O}=\Gamma(\xi, \phi \xi)$ dans $\partial^{2} X$, qui est nécessairement de $\mu_{o} \otimes \mu_{o \mid \partial^{2} X^{-}}$ mesure pleine. Or l'orbite de $\phi \xi$ suivant le stabilisateur $\Gamma_{\xi}$ ne peut s'accumuler que dans l'ensemble fini $\Lambda\left(\Gamma_{\xi}\right)$. Par conséquent, on peut trouver $\gamma \in \Gamma$ de façon que $\gamma \xi$ soit dans l'ouvert non vide de $\Lambda(\Gamma)$ qu'est le complémentaire de $\{\xi\} \cup \overline{\Gamma_{\xi} \phi \xi}$. Ainsi $(\xi, \gamma \xi) \in \partial^{2} X \backslash \mathcal{O}$, ce qui est contradictoire avec la plénitude de la mesure de $\mathcal{O}$. 
Prouvons maintenant (f). Nous nous appuierons sur la version généralisée du second lemme de Borel-Cantelli ci-dessous, dont l'idée est due à Rényi, et dont on trouvera la démonstration dans $[\mathbf{A}-\mathbf{S}]$.

LEMme 1. - Soient $M$ une mesure positive finie sur un espace $\Omega$, et $\left\{A_{t}\right\}_{t \in \mathbb{R}^{+}}$une famille de parties de $\Omega$ telle que la fonction $(t, \omega) \mapsto \mathbb{1}_{A_{t}}(\omega)$ soit mesurable. Supposons que $\int_{0}^{+\infty} M\left(A_{t}\right) d t=\infty$, et qu'il existe une constante $C>0$ telle que

$$
\int_{0}^{T} \int_{0}^{T} M\left(A_{t} \cap A_{s}\right) d t d s \leqslant C\left(\int_{0}^{T} M\left(A_{t}\right) d t\right)^{2}
$$

pour tout $T>0$ assez grand. Alors l'ensemble des $\omega \in \Omega$ tels que $\int_{0}^{+\infty} \mathbb{1}_{A_{t}}(\omega) d t=\infty$ a une $M$-mesure supérieure $\grave{a} \frac{1}{C}$.

La démonstration de (f) use de techniques voisines de celle de la première étape du théorème 4.1 plus loin. Aussi le lecteur désireux de poursuivre voudra bien consulter au début du passage cité les définitions des objets géométriques $K(z, r), \mathcal{O}_{r}^{ \pm}(a, b)$ et $\mathcal{L}_{r}(a, b)$, dont nous allons déjà nous servir. Le lemme de l'ombre 1.3 au point $x=o$ reste bien sûr valable pour les ombres par excès ou par défaut, avec un $r$ assez grand, désormais fixé. Notons alors $K=K(o, r)$.

Supposons que $\sum_{\gamma \in \Gamma} e^{-\delta(o, \gamma o)}=\infty$. Nous allons montrer ci-après les deux inégalités suivantes, valables pour $T>0$ assez grand, où le symbole $C_{i}$ désigne une constante strictement positive indépendante de $T$.

$$
\begin{gathered}
\int_{0}^{T} \int_{0}^{T} \sum_{\gamma, \phi \in \Gamma} m\left(K \cap g^{-t} \gamma K \cap g^{-t-s} \phi K\right) d t d s \leqslant C_{1}\left(\sum_{(o, \gamma o) \leqslant T} e^{-\delta(o, \gamma o)}\right)^{2} . \\
\int_{0}^{T} \sum_{\gamma \in \Gamma} m\left(K \cap g^{-t} \gamma K\right) d t \geqslant C_{2} \sum_{(o, \gamma o) \leqslant T} e^{-\delta(o, \gamma o)} .
\end{gathered}
$$

On achèvera alors la preuve en appliquant le lemme précédent avec $A_{t}=K_{\Gamma} \cap g_{\Gamma}^{-t} K_{\Gamma}$ où $K_{\Gamma}$ est le projeté de $K$ dans $S X / \Gamma$, et $M$ la mesure $m_{\Gamma}$ restreinte à $K_{\Gamma}$. La première condition exigée pour obtenir la conclusion du lemme découle de (2), et la seconde de (1) et (2) ensemble, vu que

$$
\int_{0}^{T} \int_{0}^{T} M\left(A_{t} \cap A_{s}\right) d t d s=2 \int_{0}^{T} \int_{t}^{T} M\left(A_{t} \cap A_{s}\right) d t d s \leqslant 2 \int_{0}^{T} \int_{0}^{T} M\left(A_{t} \cap A_{t+s}\right) d t d s .
$$

D'après $(\mathrm{d})$, la conclusion du lemme signifie bien que $\mu_{o}\left(\Lambda_{c}(\Gamma)\right)>0$.

Montrons donc (1). En revenant aux définitions de $m, K$ et $\mathcal{L}_{r}(a, b)$, on voit que

$$
m\left(K \cap g^{-t} \gamma K \cap g^{-t-s} \phi K\right) \leqslant \int_{\mathcal{L}_{r}(o, \phi o)} \frac{d \mu_{o}(\xi) d \mu_{o}(\eta)}{d_{o}(\xi, \eta)^{2 \delta}} r .
$$

Or $d_{o}(\xi, \eta) \geqslant e^{-r}$ lorsque $(\xi \eta)$ rencontre $B(o, r)$, et $\mathcal{L}_{r}(o, \phi o) \subset \partial X \times \mathcal{O}_{r}^{+}(o, \phi o)$. D'où

$$
m\left(K \cap g^{-t} \gamma K \cap g^{-t-s} \phi K\right) \leqslant e^{2 \delta r} r \mu_{o}\left(\mathcal{O}_{r}^{+}(o, \phi o)\right) .
$$


Enfin, en utilisant le lemme de l'ombre adapté aux ombres par excès, il vient :

$$
m\left(K \cap g^{-t} \gamma K \cap g^{-t-s} \phi K\right) \leqslant C_{3} e^{-\delta(o, \phi o)} .
$$

Mais si $K \cap g^{-t} \gamma K \cap g^{-t-s} \phi K$ n'est pas vide, on voit, par l'inégalité triangulaire, qu'alors

$$
(o, \gamma o)+(\gamma o, \phi o) \leqslant(o, \phi o)+6 r
$$

et aussi

$$
(o, \gamma o)-3 r \leqslant t \leqslant(o, \gamma o)+3 r \text { et } \quad(\gamma o, \phi o)-3 r \leqslant s \leqslant(\gamma o, \phi o)+3 r
$$

(on rappelle que $K \subset S B(o, 3 r / 2)$ ). Il résulte de tout cela que

$$
\begin{aligned}
& \int_{0}^{T} \int_{0}^{T} \sum_{\gamma, \phi \in \Gamma} m\left(K \cap g^{-t} \gamma K \cap g^{-t-s} \phi K\right) d t d s \\
& \leqslant(6 r)^{2} \sum_{\substack{(o, \gamma o) \leqslant T+3 r \\
(\gamma o, \phi o) \leqslant T+3 r}} C_{3} e^{6 \delta r} e^{-\delta(o, \gamma o)} e^{-\delta(\gamma o, \phi o)} \\
& \leqslant C_{4}\left(\sum_{(o, \gamma o) \leqslant T+3 r} e^{-\delta(o, \gamma o)}\right)^{2}
\end{aligned}
$$

(on réindexe la somme sur $\phi$ en remplaçant ce dernier par $\gamma \phi$ ). Comme

$$
\sum_{T<(o, \gamma o) \leqslant T+3 r} e^{-\delta(o, \gamma o)}
$$

est bornée, grâce à la majoration de la fonction orbitale de $\Gamma$ vue à la suite du lemme 1.3 , et comme $\sum e^{-\delta(o, \gamma o)}$ diverge, l'inégalité (1) s'ensuit.

Montrons enfin (2) de façon similaire. Si $\gamma \in \Gamma$ est tel que

$$
3 r \leqslant(o, \gamma o) \leqslant T-3 r
$$

on voit, en examinant plus attentivement la définition de $K=K(o, r)$, qu'alors

$$
\begin{aligned}
\int_{0}^{T} m\left(K \cap g^{-t} \gamma K\right) d t & =\int_{\mathcal{L}_{r}(o, \gamma o)} \frac{d \mu_{o}(\xi) d \mu_{o}(\eta)}{d_{o}(\xi, \eta)^{2 \delta}} \int_{-r / 2}^{r / 2} \int_{0}^{T} \mathbb{1}_{\gamma K}\left(g^{s+t} o_{\xi \eta}\right) d s d t \\
& =r^{2} \int_{\mathcal{L}_{r}(o, \gamma o)} \frac{d \mu_{o}(\xi) d \mu_{o}(\eta)}{d_{o}(\xi, \eta)^{2 \delta}}
\end{aligned}
$$

ce qui est plus grand que $r^{2} \mu_{o}\left(\mathcal{O}_{r}^{-}(\gamma o, o)\right) \mu_{o}\left(\mathcal{O}_{r}^{-}(o, \gamma o)\right)$, puisque

$$
\mathcal{L}_{r}(o, \gamma o) \supset \mathcal{O}_{r}^{-}(\gamma o, o) \times \mathcal{O}_{r}^{-}(o, \gamma o)
$$

et que le diamètre de $d_{x}$ est 1 . Pourvu seulement que nous ayions pris $r$ assez grand avant de commencer, nous savons que $\mu_{o}\left(\mathcal{O}_{r}^{-}(\gamma o, o)\right) \geqslant C_{5}$, comme on aura pu le voir dans la preuve du lemme de l'ombre 1.3 (ici adapté aux ombres par défaut). Qui plus est, ce même lemme nous assure que $\mu_{o}\left(\mathcal{O}_{r}^{-}(o, \gamma o)\right) \geqslant C_{6} e^{-\delta(o, \gamma o)}$. En définitive, on a

$$
\int_{0}^{T} \sum_{\gamma \in \Gamma} m\left(K \cap g^{-t} \gamma K\right) d t \geqslant C_{7} \sum_{3 r \leqslant(o, \gamma o) \leqslant T-3 r} e^{-\delta(o, \gamma o)} .
$$

On en déduit (2) par l'argument terminant la preuve de (1). 
Prouvons à présent $(\mathrm{g})$, ce qui nécessitera le lemme de dérivation de la mesure $\mu_{o}$ ci-dessous. Quand $X$ est l'espace hyperbolique, les ombres à l'infini sont des boules euclidiennes et l'énoncé suivant, alors contenu dans un théorème classique, peut être employé sans autre forme de procès, ce qui n'est plus permis dès que l'on se place en courbure variable - ce point a été négligé dans [YY]-, où subsiste cependant ce lemme 2 , que nous montrerons ci-après. Pour $r>0$, on notera $\Lambda(r)$ l'ensemble des points de $\partial X$ appartenant à une infinité d'ombres distinctes $\mathcal{O}_{r}(o, \gamma o)(\gamma \in \Gamma)$; observons que $\Lambda_{c}(\Gamma)$ est la réunion croissante des $\Lambda(r)$ quand $r$ croît indéfiniment.

LEMme 2. - Dès que $r>0$ est assez grand, pour toute fonction $h \in L^{1}\left(\mu_{o}\right)$, pour $\mu_{o}$-presque tout $\xi \in \Lambda(r)$, on a

$$
\frac{1}{\mu_{o}\left(\mathcal{O}_{r}(o, \gamma o)\right)} \int_{\mathcal{O}_{r}(o, \gamma o)} \varphi d \mu_{o} \longrightarrow \varphi(\xi)
$$

quand $(o, \gamma o) \rightarrow+\infty$ avec $\mathcal{O}_{r}(o, \gamma o) \ni \xi$

Terminons d'abord la preuve de (g). Supposons que $\mu_{o}\left(\Lambda_{c}(\Gamma)\right)>0$. Nous allons en fait montrer que si un borélien $A \subset \Lambda_{c}(\Gamma)$ de $\mu_{o}$-mesure non nulle est invariant par $\Gamma$, alors $\mu_{o}(A)=1$. Prenons un tel $A$ (par exemple $\Lambda_{c}(\Gamma)$ ), et $r>0$ assez grand pour que $\mu_{o}(A \cap \Lambda(r))>0$ et que les conclusions du lemme 2 ci-dessus et du lemme 1.3 (avec $x=o$ ) soient toutes deux valides. Notons $B=\partial X \backslash A$. Une application du lemme 2 avec $h=\mathbb{1}_{B}$ et $\xi \in A \cap \Lambda(r)$ nous procure une suite $\left\{\gamma_{n}\right\} \subset \Gamma$ telle que

$$
\left(o, \gamma_{n} o\right) \longrightarrow \infty \quad \text { et } \quad \frac{\mu_{o}\left(\mathcal{O}_{r}\left(o, \gamma_{n} o\right) \cap B\right)}{\mu_{o}\left(\mathcal{O}_{r}\left(o, \gamma_{n} o\right)\right)} \longrightarrow 0 \quad \text { quand } n \longrightarrow \infty
$$

Or le quotient ci-dessus est proportionnel à $\mu_{\gamma_{n} o}\left(\mathcal{O}_{r}\left(o, \gamma_{n} o\right) \cap B\right)$, ce que l'on voit en utilisant les lemmes 1.2 (pour le numérateur) et 1.3 (pour le dénominateur). Compte tenu de l'invariance de $B$ par $\Gamma$, la quantité $\mu_{o}\left(\mathcal{O}_{r}\left(\gamma_{n}^{-1} o, o\right) \cap B\right)$ est égale à la précédente, et tend donc vers 0 . En prenant des valeurs de $r$ arbitrairement grandes, on obtient une suite d'ouverts $\Omega_{k}$ de $\partial X$ telle que le diamètre de $\partial X \backslash \Omega_{k}$ tend vers 0 et $\mu_{o}\left(\Omega_{k} \cap B\right) \rightarrow 0$ quand $k \rightarrow \infty$. Quitte à extraire, on peut supposer que $\partial X \backslash \Omega_{k}$ converge au sens de Hausdorff vers un point $\zeta$; alors $\mu_{o}(B \backslash\{\zeta\})=0$. En prenant $\gamma \in \Gamma$ tel que $\gamma \zeta \neq \zeta$, en utilisant encore l'invariance de $B$ par $\gamma$, on aboutit enfin à $\mu_{o}(B)=0$, ce qu'il fallait montrer.

Complétons cette démonstration par celle du lemme 2. Pour $h \in L^{1}\left(\mu_{o}\right)$, introduisons la fonction (dite maximale) $h^{*}$ bien définie en tout point $\xi \in \Lambda(r)$ par

$$
\varphi^{*}(\xi)=\lim _{t \rightarrow+\infty} \sup \frac{1}{\mu_{o}\left(\mathcal{O}_{r}(o, \gamma o)\right)} \int_{\mathcal{O}_{r}(o, \gamma o)} \varphi d \mu_{o}
$$

où la borne supérieure est prise sur les $\gamma \in \Gamma$ tels que $(o, \gamma o)>t$ et $\xi \in \mathcal{O}_{r}(o, \gamma o)$; posons $\varphi^{*}=0$ sur $\partial X \backslash \Lambda(r)$. Par un argument simple que le lecteur pourra retrouver 
à la fin de la preuve du théorème 2.2, on sait que la conclusion désirée découle de l'inégalité $(*)$ suivante, où $C>0$ ne dépend pas de $h$ :

$$
\text { pour tout } \varepsilon>0, \quad \mu_{o}\left(\left\{\varphi^{*}>\varepsilon\right\}\right) \leqslant \frac{C}{\varepsilon} \int_{\partial X} \varphi d \mu_{o} .
$$

Fixons $\varepsilon>0$ et montrons $(*)$. Nous disposons de $G \subset \Gamma$ tel que

$$
\left\{\varphi^{*}>\varepsilon\right\} \subset \bigcup_{\gamma \in G} \mathcal{O}_{r}(o, \gamma o) \quad \text { et } \quad \frac{1}{\mu_{o}\left(\mathcal{O}_{r}(o, \gamma o)\right)} \int_{\mathcal{O}_{r}(o, \gamma o)} \varphi d \mu_{o}>\varepsilon
$$

pour tout $\gamma \in G$. Notons $G=\left\{\gamma_{i}\right\}_{i \in \mathbb{N}}$ avec $\left(o, \gamma_{i} o\right) \leqslant\left(o, \gamma_{i+1} o\right)$ pour tout $i$. Définissons par récurrence $i_{0}=0$, puis

$$
i_{k+1}=\min \left\{i>i_{k} \mid \mathcal{O}_{r}\left(o, \gamma_{i} o\right) \cap \cup_{j \leqslant k} \mathcal{O}_{r}\left(o, \gamma_{i_{j}} o\right)=\varnothing\right\} .
$$

Si $i_{k}<i<i_{k+1}$ pour un $k$, alors $\mathcal{O}_{r}\left(o, \gamma_{i} o\right) \cap \mathcal{O}_{r}\left(o, \gamma_{i_{j}} o\right) \neq \varnothing$ pour un $j \leqslant k$, et l'on dispose d'un rayon géodésique partant de $o$ qui traverse $B\left(\gamma_{i_{j}} o, r\right)$ et $B\left(\gamma_{i} o, r\right)$; s'il les rencontre dans cet ordre, alors $\mathcal{O}_{r}\left(o, \gamma_{i} o\right) \subset \mathcal{O}_{3 r}\left(o, \gamma_{i_{j}} o\right)$ par un simple argument de géométrie hyperbolique; sinon, on voit que $B\left(\gamma_{i} o, r\right) \subset B\left(\gamma_{i_{j}} o, 5 r\right)$ du fait que $\left(o, \gamma_{i} o\right) \geqslant\left(o, \gamma_{i_{j}} o\right)$; on en conclut dans tous les cas que $\mathcal{O}_{r}\left(o, \gamma_{i} o\right) \subset \mathcal{O}_{5 r}\left(o, \gamma_{i_{j}} o\right)$. Ainsi, en notant $G^{*}=\left\{\gamma_{i_{j}}, j \in \mathbb{N}\right\}$, les ombres $\mathcal{O}_{r}(o, \gamma o), \gamma \in G^{*}$ sont deux à deux disjointes, et nous venons d'établir que

$$
\bigcup_{\gamma \in G} \mathcal{O}_{r}(o, \gamma o) \subset \bigcup_{\gamma \in G^{*}} \mathcal{O}_{5 r}(o, \gamma o) .
$$

D'après le lemme de l'ombre 1.3 , on voit que pour $r$ assez grand, on a

$$
\mu_{o}\left(\mathcal{O}_{5 r}(o, \gamma o)\right) \leqslant C_{0} \mu_{o}\left(\mathcal{O}_{r}(o, \gamma o)\right)
$$

pour une constante $C_{0}$ indépendante de $\gamma \in \Gamma$. Il résulte de tout cela que

$$
\begin{aligned}
\mu_{o}\left(\left\{\varphi^{*}>\varepsilon\right\}\right) \leqslant \sum_{\gamma \in G^{*}} \mu_{o}\left(\mathcal{O}_{5 r}(o, \gamma o)\right) & \leqslant C_{0} \sum_{\gamma \in G^{*}} \mu_{o}\left(\mathcal{O}_{r}(o, \gamma o)\right) \\
& \leqslant \frac{C_{0}}{\varepsilon} \sum_{\gamma \in G^{*}} \int_{\mathcal{O}_{r}(o, \gamma o)} \varphi d \mu_{o} \leqslant \frac{C_{0}}{\varepsilon} \int_{\partial X}|\varphi| d \mu_{o},
\end{aligned}
$$

ce qui termine la preuve du lemme 2 et de $(\mathrm{g})$.

Prouvons enfin (h), en reprenant un argument célèbre de Hopf (cf. [Ho]). Comme dans [Sull1], introduisons une fonction $\widetilde{\rho}(u)=e^{-4 \delta(u, \Gamma o)}(u \in S X)$; elle induit une fonction $\rho$ sur $S X / \Gamma$. Il est aisé de vérifier que $m(S B(o, r)) \leqslant 2 r e^{2 \delta r}$; or en appelant $D \subset X$ le domaine de Dirichlet pour $\Gamma$ centré en $o$, on a $\widetilde{\rho}(u)=e^{-4 \delta(u, o)}$ pour $u \in S \bar{D}$; l'intégrale par rapport à $m$ de $\widetilde{\rho}$ sur $[S B(o, r+1) \backslash S B(o, r)] \cap S \bar{D}$ est donc majorée par une constante fois $e^{-\delta r}$. Il s'ensuit que $\rho \in L^{1}\left(m_{\Gamma}\right)$. D'autre part, on vérifie facilement que pour tous $u$ et $v$ dans $S X$ tels que

$$
(u, v) \leqslant 1, \quad|\widetilde{\rho}(v)-\widetilde{\rho}(u)| \leqslant 4 \delta e^{4 \delta}(u, v) \widetilde{\rho}(u) .
$$


Comme $\rho$ est continue et strictement positive, on a

$$
\int_{0}^{ \pm \infty} \widetilde{\rho}\left(g^{t} u\right) d t= \pm \infty
$$

pour tout $u$ tel que $g^{ \pm \infty} \in \Lambda_{c}(\Gamma)$. Considérons $h \in C_{c}(S X / \Gamma)$ que l'on relève en $\widetilde{h}$ sur $S X$, et définissons $h^{*}\left(\operatorname{resp} . h_{*}\right)$ sur $\left(g^{+\infty}\right)^{-1} \Lambda_{c}(\Gamma)\left(\operatorname{resp} .\left(g^{-\infty}\right)^{-1} \Lambda_{c}(\Gamma)\right)$ par

$$
h^{*}=\limsup _{T \rightarrow+\infty} \frac{\int_{0}^{T} \widetilde{h} \circ g^{t} d t}{\int_{0}^{T} \tilde{\rho} \circ g^{t} d t} \quad \text { et } \quad h_{*}=\limsup _{T \rightarrow+\infty} \frac{\int_{0}^{T} \widetilde{h} \circ g^{-t} d t}{\int_{0}^{T} \widetilde{\rho} \circ g^{-t} d t} \text {. }
$$

On vérifie aisément, à l'aide des propriétés de continuités de $\widetilde{h}$ et $\widetilde{\rho}$ indiquées plus haut, que $h^{*}$ (resp. $h_{*}$ ) est constante sur les horosphères stables (resp. instables) basées dans $\Lambda_{c}(\Gamma)$. De plus, elles sont toutes deux bornées et invariantes (stricto sensu) par $\Gamma$ et $g^{t}$. Elles induisent ainsi chacune une fonction borélienne sur $\Lambda_{c}(\Gamma)$. Supposons $\left(S X / \Gamma,\left\{g_{\Gamma}^{t}\right\}_{t \in \mathbb{R}}, m_{\Gamma}\right)$ complètement conservatif, ou encore $\mu_{o}\left(\Lambda_{c}(\Gamma)\right)=1$. Alors $h^{*}$ et $h_{*}$ sont égales $m$-presque partout, d'après le théorème de Hopf (voir [Ho], [Kre]). Avec ce qui précède, on conclut qu'elles sont $m$-essentiellement constantes. Ainsi les moyennes ergodiques

$$
\frac{\int_{0}^{T} h \circ g_{\Gamma}^{-t} d t}{\int_{0}^{T} \rho \circ g_{\Gamma}^{-t} d t}
$$

convergent $m_{\Gamma}$-presque partout vers une fonction constante quand $|T| \rightarrow \infty$, ce pour toute $h$ dans $C_{c}(S X / \Gamma)$, puis dans $L^{1}\left(m_{\Gamma}\right)$, grâce encore au théorème de Hopf, ce qui signifie l'ergodicité de $\left(S X / \Gamma,\left\{g_{\Gamma}^{t}\right\}_{t \in \mathbb{R}}, m_{\Gamma}\right)$. Ainsi se termine la preuve de (h), et avec elle celle du théorème.

Démontrons pour finir le corollaire 1.8. Supposons $\Gamma$ de type divergent. L'argument d'unicité sera repris de [Sull1]. Si $\mu$ et $\mu^{\prime}$ sont deux densités conformes de dimension $\delta(\Gamma)$ et invariantes par $\Gamma$, il en est alors de même de $\nu=\frac{1}{2}\left(\mu+\mu^{\prime}\right)$. Or $\mu_{o}$ est absolument continue par rapport à $\nu_{o}$, et l'invariance par $\Gamma$ de $\mu$ et de $\nu$ entraîne celle de $d \mu_{o} / d \nu_{o}$ modulo $\nu_{o}$. On sait en particulier que $\left(\partial X, \Gamma, \nu_{o}\right)$ est ergodique, et par conséquent $d \mu_{o} / d \nu_{o}$ est essentiellement constante, égale à 1 , d'où $\mu=\nu=\mu^{\prime}$.

La non-atomicité de $\mu$ découle du lemme de l'ombre et du fait que $\mu_{o}\left(\Lambda_{c}(\Gamma)\right)=1$; l'ensemble des points fixes de $\Gamma$ dans $S X$ est invariant par $\Gamma$ et par le flot géodésique, de plus fermé et différent du support de $m$, donc $m$-négligeable par ergodicité du flot géodésique.

Prouvons enfin que $\mu_{o}\left(\Lambda_{M}(\Gamma)\right)=1$. Soit $\mathcal{B}$ une base dénombrable de la topologie du support de $m$ dans $S X$, c'est-à-dire de $\left(\partial^{2} X \cap(\Lambda(\Gamma) \times \Lambda(\Gamma)) \times \mathbb{R}\right.$. Pour $V \in \mathcal{B}$, soit $\mathcal{M}(V)$ l'ensemble des $u \in S X$ tels que $g^{t_{i}} u \in \Gamma V$ pour une suite $t_{i}$ tendant vers $+\infty$. Le borélien $\mathcal{M}(V)$ est invariant par $\Gamma$ et par le flot géodésique. Grâce à la conservativité de $\left(S X / \Gamma,\left\{g_{\Gamma}^{t}\right\}_{t \in \mathbb{R}}, m_{\Gamma}\right)$, on sait que $\mathcal{M}(V) \supset \Gamma V$ modulo $m$. L'ergodicité du flot géodésique montre ensuite que $\mathcal{M}(V)$ est de $m$-mesure pleine (on 
a $m(V)>0)$. Or $g^{+\infty}\left(\bigcap_{V \in \mathcal{B}} \mathcal{M}(V)\right)=\Lambda_{M}(\Gamma)$ par la définition même de $\Lambda_{M}(\Gamma)$, ce qui emporte la conclusion.

\section{F. Groupes géométriquement finis}

Cette section est consacrée aux groupes d'isométries discrets les plus simples que l'on conçoive du point de vue géométrique. On songe en premier lieu aux groupes cocompacts, c'est-à-dire dont le quotient de $X$ associé est compact; l'ensemble limite d'un tel groupe est le bord à l'infini tout entier. Les propriétés de ces groupes ont d'ordinaire leur homologue dans la classe plus générale des groupes $\Gamma$ convexe-cocompacts, qui par définition agissent de façon cocompacte sur l'enveloppe $\mathcal{E}$ de $\Lambda(\Gamma)$ dans $X$, définie comme étant la réunion des géodésiques à extrémités dans $\Lambda(\Gamma)$. Faisons observer que $\mathcal{E}$ n'est pas précisément convexe; néanmoins, si $X$ est une variété à courbure pincée, l'enveloppe convexe de $\Lambda(\Gamma)$ est contenue dans un voisinage uniforme de $\mathcal{E}$ (voir [Bow2], paragraphe 2.5).

Supposons que $\Gamma$ soit convexe-cocompact. Soit $m_{\Gamma}$ la mesure de BMS associée à une densité conforme invariante par $\Gamma$ et de support $\Lambda(\Gamma)$, comme l'on en sait exister d'après le théorème 1.1. Vu que son support est le quotient par $\Gamma$ de

$$
\left(\partial^{2} X \cap(\Lambda(\Gamma) \times \Lambda(\Gamma)) \times \mathbb{R} \subset S X\right.
$$

lequel quotient est compact, la mesure $m_{\Gamma}$ est finie. Aussi les groupes convexecocompacts appartiennent-ils à cette classe remarquable présentée suite au corollaire 1.8. En fait, les groupes convexe-cocompacts sont précisément les groupes admettant une mesure de BMS à support compact. Notons que ce sont par ailleurs les groupes dont tous les points limites sont coniques; on en verra d'autres caractérisations après la proposition 1.10 .

Nous allons ci-après introduire une classe de groupes plus large que la précédente. Il s'agira des groupes dits géométriquement finis, dont les multiples caractérisations sont bien connues dans le cadre du disque hyperbolique; que l'on hausse seulement la dimension de l'espace, et déjà pointent quelques complications, dont un tour d'horizon a été mené à bien dans [Bow1]; cette étude a été poursuivie en courbure variable pincée dans [Bow2], où l'on est contraint de renoncer à la caractérisation en termes de géométrie d'un domaine fondamental. Comme les méthodes de [Bow2] ressortissent par trop à la géométrie riemannienne (emploi du volume...), nous proposons ci-dessous une analyse de la notion de finitude géométrique dans la généralité des espaces CAT $(-1)$, ce qui, autant que nous le sachions, n'a pas encore été examiné. Nous montrerons que l'équivalence des deux caractérisations fondamentales reste valable (proposition 1.10); pour finir, nous aborderons la question de la finitude d'une mesure de BMS pour les groupes décrits en nous référant à [D-O-P]. 
Un point $p$ (dans $\Lambda(\Gamma)$ ) est dit parabolique si son stabilisateur $\Gamma_{p}$ dans $\Gamma$ est un groupe (élémentaire) parabolique (cf. $1 \mathrm{~A})$; on a alors $\Lambda\left(\Gamma_{p}\right)=\{p\}$. Si en outre $\Gamma_{p}$ agit de façon cocompacte sur $\Lambda(\Gamma) \backslash\{p\}$, alors $p$ sera dit parabolique borné. L'ensemble des points fixes paraboliques bornés de $\Gamma$ sera noté $\Lambda_{p b}(\Gamma)$. Lorsque $X$ est une surface, tout point parabolique est borné, tandis qu'en dimension supérieure, ces deux catégories se côtoient. Nous donnerons plus loin une preuve commune des énoncés 1.9 et 1.10.

Le lemme suivant établit l'existence d'horoboules précisément invariantes pour un point parabolique borné. En courbure pincée, ce fait est même avéré pour tout point parabolique, selon le Lemme de Margulis (voir [Bow2]); ici, la compacité introduite par l'hypothèse que le point soit borné permet de pallier l'absence de borne inférieure générale sur la courbure.

Lemme 1.9. - Soit p un point parabolique borné de $\Gamma$. Il existe alors une horoboule $H$ basée en $p$ telle que les images de $H$ par $\Gamma$ soient deux à deux disjointes ou confondues (une telle horoboule est dite précisément invariante par $\Gamma_{p}$ ). En corollaire, il apparaît que $\Lambda_{h}(\Gamma) \cap \Lambda_{p b}(\Gamma)=\varnothing$.

Nous dirons que $\Gamma$ est géométriquement fini lorsque $\Lambda(\Gamma)=\Lambda_{h}(\Gamma) \cup \Lambda_{p b}(\Gamma)$. Ce n'est pas là la définition courante, qui stipule plutôt que $\Lambda(\Gamma)=\Lambda_{c}(\Gamma) \cup \Lambda_{p b}(\Gamma)$, mais leur équivalence a été déjà signalée dans [Dal] pour ce qui est des variétés; au surplus, cette présentation paraîtra plus naturelle à qui étudiera la preuve plus bas. L'assertion (iii) ci-dessous décrit l'action caractéristique d'un groupe géométriquement fini sur $X$. On y retrouve l'image familière d'un quotient $X / \Gamma$ composé d'une partie compacte, d'un nombre fini de bouts hyperboliques et d'un nombre fini de cornes paraboliques.

Proposition 1.10. - Les trois assertions suivantes sont équivalentes, et signifient que $\Gamma$ est géométriquement fini :

(i) $\Lambda(\Gamma)=\Lambda_{h}(\Gamma) \cup \Lambda_{p b}(\Gamma)$.

(ii) $\Lambda(\Gamma)=\Lambda_{c}(\Gamma) \cup \Lambda_{p b}(\Gamma)$.

(iii) $\Lambda_{p b}(\Gamma)$ est la réunion d'un nombre fini ou nul $k$ d'orbites distinctes $\Gamma p_{1}, \ldots, \Gamma p_{k}$, et chaque $p_{i}$ est la base d'une horoboule $H_{i}$, de telle sorte que les images par $\Gamma$ des horoboules $H_{1}, \ldots, H_{k}$ sont deux à deux disjointes ou confondues, et que le quotient de $\mathcal{E} \backslash\left(\Gamma H_{1} \cup \cdots \cup \Gamma H_{k}\right)$ par $\Gamma$ est compact, où $\mathcal{E}$ désigne la réunion des géodésiques à extrémités dans $\Lambda(\Gamma)$.

Notons maintenant que $\Gamma$ est convexe-cocompact si et seulement si $\Lambda(\Gamma)=\Lambda_{h}(\Gamma)$, ou encore $\Lambda(\Gamma)=\Lambda_{c}(\Gamma)$, et que les groupes convexes-cocompacts sont, en dernière analyse, exactement les groupes géométriquement finis ne contenant aucun sous-groupe parabolique.

Démonstration. - Pour montrer les énoncés 1.9 et 1.10 , nous nous appuierons sur le lemme suivant, qui contient le premier de ceux-là. 
LEMME 1. - Si p et $p^{\prime}$ sont dans $\Lambda_{p b}(\Gamma)$ et si $H^{\prime}$ est une quelconque horoboule basée en $p^{\prime}$, il existe alors une horoboule $H$ basée en $p$, telle que pour tout $\gamma \in \Gamma$, on ait $\gamma H \cap H^{\prime}=\varnothing$, à moins que $\gamma p=p^{\prime}$.

Prouvons le lemme 1 par l'absurde, en supposant que pour tout $n \in \mathbb{N}$, l'on ait quelque $\gamma_{n} \in \Gamma$ tel que $\gamma_{n} p \neq p^{\prime}$ et $\gamma_{n} H_{n} \cap H^{\prime} \neq \varnothing$, où $H_{0}$ est une quelconque horoboule fixée basée en $p$, et $H_{n}$ l'horoboule contenue dans $H_{0}$ de bord à distance $n$ de celui de $H_{0}$. Vu que $H_{n} \cap \gamma_{n}^{-1} H^{\prime} \neq \varnothing$, la géodésique $\left(p \gamma_{n}^{-1} p^{\prime}\right)$ rencontre le bord de $\gamma_{n}^{-1} H^{\prime}$ en un point $x_{n} \in H_{n}$; soit aussi $z_{n}$ le point d'intersection de cette géodésique avec $\partial H_{0}$. Comme $\Gamma_{p}$ agit de façon cocompacte sur $\Lambda(\Gamma) \backslash\{p\}$ et partant sur $\partial H_{0} \cap \bigcup_{\xi \in \Lambda(\Gamma) \backslash\{p\}}(p \xi)$, on peut supposer que $z_{n}$ demeure dans un certain compact, quitte à composer $\gamma_{n}$ à droite par un élément de $\Gamma_{p}$ et à remplacer $x_{n}$ par son antécédent par ce dernier, donc sans toucher à $\gamma_{n} x_{n}$. Il est alors facile de vérifier, par comparaison avec le demi-plan hyperbolique, que pour $\pi \in \Gamma_{p}$ donné, on a $\left(x_{n}, \pi x_{n}\right) \leqslant e^{-n}\left(z_{n}, \pi z_{n}\right)=O\left(e^{-n}\right)$. D'autre part, comme $\Gamma_{p^{\prime}}$ agit de façon cocompacte sur $\partial H^{\prime} \cap \bigcup_{\xi \in \Lambda(\Gamma) \backslash\left\{p^{\prime}\right\}}\left(p^{\prime} \xi\right)$, on peut de même supposer que $\gamma_{n} x_{n}$ ne quitte pas un certain compact, quitte à modifier $\gamma_{n}$ sans déplacer $x_{n}$. On peut alors trouver une suite $n_{i} \rightarrow+\infty$ telle que $\gamma_{n_{i}} x_{n_{i}}$ converge vers un point $y \in X$. Considérons $\pi \in \Gamma_{p}$ : on a vu plus haut que $\left(x_{n_{i}}, \pi x_{n_{i}}\right) \rightarrow 0$, d'où $\left(y, \gamma_{n_{i}} \pi \gamma_{n_{i}}^{-1} y\right) \rightarrow 0$, et par suite $\gamma_{n_{i}} \pi \gamma_{n_{i}}^{-1} \in \Gamma_{y}$ dès que $i$ est assez grand ( $\Gamma$ est discret). Mais $\Gamma_{y}$ étant fini, on en déduit que $\Gamma_{p}$ l'est également, et que $\gamma_{n_{i}} \Gamma_{p} \gamma_{n_{i}}^{-1} \subset \Gamma_{y}$ pour $i$ assez grand, soit $\Gamma_{p} \subset \Gamma_{\gamma_{n_{i}}^{-1} y}$, ce qui est absurde parce que $\Gamma_{p}$ est parabolique et ne fixe par conséquent que $p$.

Le lemme 2 décrit l'action du stabilisateur $\Gamma_{p}$ d'un point parabolique borné $p$ sur $\mathcal{E}$.

Lemme 2. - Soient $p \in \Lambda_{p b}(\Gamma)$, et $H$ une horoboule basée en $p$. Alors le quotient de $[\mathcal{E} \cup(\Lambda(\Gamma) \backslash\{p\})] \backslash H$ par $\Gamma_{p}$ est compact.

En effet, prenons un domaine $F$ fondamental localement fini pour l'action de $\Gamma_{p}$ sur $\Lambda(\Gamma) \backslash\{p\}$. Alors $\bar{F} \subset \Lambda(\Gamma) \backslash\{p\}$, vu que $p \in \Lambda_{p b}(\Gamma)$. Soit $\widehat{F}$ l'ensemble des $x \in X$ tels que la boule $B(x, \operatorname{Argcosh} \sqrt{2})$ rencontre la réunion des géodésiques dont l'une des extrémités est $p$ et l'autre dans $\bar{F}$. Considérons $a$ et $b$ distincts dans $\Lambda(\Gamma) \backslash\{p\}=\Gamma_{p} \bar{F}$. Par comparaison avec le demi-plan hyperbolique, on voit que tout point de $(a b)$ est à distance au plus $\operatorname{Argcosh} \sqrt{2}$ de $(p a) \cup(p b)$. Par conséquent, $\mathcal{E} \subset \Gamma_{p} \widehat{F}$. Or il est clair que $\widehat{F} \backslash H$ est relativement compact dans $\bar{X} \backslash\{p\}$, ce qui emporte la conclusion.

Démontrons enfin la proposition 1.10. Les implications (iii) $\Rightarrow$ (ii) $\Rightarrow$ (i) sont évidentes. Supposons (i) et montrons alors (iii). Soient $p_{1}, p_{2} \ldots$ des représentants de chaque orbite suivant $\Gamma$ dans $\Lambda_{p b}(\Gamma)$ (ce dernier peut être vide). En utilisant le lemme 1 , on peut trouver pour chaque $p_{i}$ une horoboule $H_{i}$ basée en $p_{i}$ de telle sorte que les images des horoboules fermées $\overline{H_{1}}, \ldots$ soient deux à deux disjointes ou confondues. Soit $D$ le domaine de Dirichlet pour $\Gamma$ centré en 0 ( $c f .1 \mathrm{C})$. Nous allons prouver la compacité de $\bar{D} \cap \mathcal{E} \backslash\left(\Gamma H_{1} \cup \ldots\right)$, ce qui entraînera en sus que le nombre 
d'horoboules $H_{1}, \ldots$ est fini, autrement dit que le nombre d'orbites dans $\Lambda_{p b}(\Gamma)$ est fini. Soit $\left\{x_{n}\right\}$ une suite de points de $\bar{D} \cap \mathcal{E}$ non bornée (s'il en existe). Quitte à extraire, on peut supposer que $\left\{x_{n}\right\}$ converge vers un point $\xi$, nécessairement situé dans $\Lambda(\Gamma)=\overline{\mathcal{E}} \cap \partial X$. Pour tout $\gamma \in \Gamma$, on a $\left(x_{n}, o\right) \leqslant\left(x_{n}, \gamma o\right)$ car $x_{n} \in \bar{D}$, et par suite $\beta_{\xi}(o, \gamma o) \leqslant 0$. Ainsi $\xi \notin \Lambda_{h}(\Gamma)$, et donc $\xi \in \Lambda_{p b}(\Gamma)$ selon (i); on peut supposer que $\xi=p_{1}$ par exemple. Or $D$ est contenu dans le domaine $D_{1}$ de Dirichlet pour $\Gamma_{p_{1}}$ centré en $o$, et d'après le lemme 2 , on sait que $D_{1} \backslash H_{1}$ est isolé de $p_{1}$, compte tenu du fait que $D_{1}$ est localement fini. Comme $D \ni x_{n} \rightarrow p_{1}$, on en déduit que $x_{n} \in H_{1}$ pour $n$ assez grand. Ceci achève de prouver que $\bar{D} \cap \mathcal{E} \backslash\left(\Gamma H_{1} \cup \ldots\right)$ est compact.

La structure des groupes géométriquement finis que l'on vient de présenter offre une certaine simplicité du point de vue géométrique. Cependant, tout au long du présent article, nous mènerons l'étude des groupes principalement par la voie des mesures de BMS associées, et de ce point de vue différent, les groupes les plus accessibles sont sans conteste ceux qui admettent une telle mesure qui soit finie. Dès lors se pose la question de savoir quels liens existent entre ceux-ci et ceux-là, entre ces deux espèces de finitude. Lorsque $X$ est un espace hyperbolique (courbure constante), il est bien connu que les groupes géométriquement finis admettent une mesure de BMS finie (voir [Sull3]) en particulier, ils sont de type divergent. Le problème de la réciproque a même été suggéré dans [Sull2] : en fait, cette dernière a été très largement infirmée, y compris en dimension deux, depuis que de larges familles de groupes fuchsiens admettant une mesure de BMS finie, tout en étant eux-mêmes géométriquement infinis, ont été bâties, par des voies toutes différentes, dans $[\mathbf{A n c}]$ et $[\mathbf{P e i}]$, auxquels nous renvoyons le lecteur. La situation en courbure variable pincée a été étudiée dans $[\mathbf{D}-\mathbf{O}-\mathbf{P}]$, et paraît plus confuse, du moins plus variée : loin de toujours posséder une mesure de BMS finie, un groupe géométriquement fini peut même s'avérer de type convergent. Les critères de divergence et de finitude élaborés dans $[\mathbf{D}-\mathbf{O}-\mathbf{P}]$ ne requièrent point de borne inférieure sur la courbure, et se transposent avec leurs preuves dans le cadre des espaces CAT $(-1)$, à quelques formulations près. Nous aurons besoin du critère de finitude suivant à propos du théorème 5.2, dont la preuve présente d'ailleurs un point commun avec celle de celui-là (on y prouve une finitude de mesure à partir de celle de la série ci-dessous).

THÉorÈme 1.11 ([D-O-P], théorème B). - Supposons que $\Gamma$ soit géométriquement fini, et soit $m_{\Gamma}$ une mesure de Bowen-Margulis-Sullivan associée à une densité conforme de dimension $\delta(\Gamma)$. Alors $m_{\Gamma}$ est finie si et seulement si $\Gamma$ est de type divergent et si pour tout $p \in \Lambda_{p b}(\Gamma)$, pour tout $x \in X$, la série

$$
\sum_{\pi \in \Gamma_{p}}(x, \pi x) e^{-\delta(\Gamma)(x, \pi x)}
$$

converge. 
Avec le critère de divergence ([D-O-P], théorème $\mathrm{A})$, on obtient un corollaire remarquable : supposons que $\Gamma$ soit géométriquement fini, et que pour tout $p \in \Lambda_{p b}(\Gamma)$, l'on ait $\delta\left(\Gamma_{p}\right)<\delta(\Gamma)$; alors la mesure $m_{\Gamma}$ est finie.

\section{G. Distances et mesures sur les horosphères}

Considérons une horosphère $H$ dans $X$ basée en $\zeta \in \partial X$. On dispose d'une projection $P_{H}$ de $\partial X \backslash\{\zeta\}$ dans $H$ qui à $\xi \in \partial X \backslash\{\zeta\}$ associe le point d'intersection de $H$ et de la géodésique $(\zeta \xi)$. Dans $[\mathbf{H}-\mathbf{P 1}]$ a été construite une distance sur $\partial X \backslash\{\zeta\}$, dite d'Euclide-Cygan, ou encore de Hämenstadt, que nous allons reprendre en la projetant dans $H$. Dans la généralité des espaces CAT $(-1)$, il se peut que $P_{H}$ ne soit pas réellement surjective mais nous nous intéressons à l'image de $P_{H}$ plutôt qu'à $H$ tout entière. Notons que $P_{H}$ est un homéomorphisme sur son image.

Étant donnés $\xi$ et $\eta$ dans $\partial X \backslash\{\zeta\}$, notons $\xi_{t}$ et $\eta_{t}$ les géodésiques issues de $\zeta$, finissant respectivement en $\xi$ et en $\eta$, et telles que $\xi_{0} \in H$ et $\eta_{0} \in H$ (autrement dit $\xi_{0}=P_{H} \xi$ et $\left.\eta_{0}=P_{H} \eta\right)$. Définissons alors

$$
d_{H}\left(P_{H} \xi, P_{H} \eta\right)=\lim _{t \rightarrow+\infty} e^{-t+\frac{1}{2}\left(\xi_{t}, \eta_{t}\right)},
$$

l'existence de cette limite s'obtenant par un argument de type Cauchy. On vérifie aisément que si $r(t)$ est un rayon géodésique finissant en $\zeta$ et tel que $r(0) \in H$, alors $d_{H}\left(P_{H} \xi, P_{H} \eta\right)=\lim _{t \rightarrow+\infty} e^{t} d_{r(t)}(\xi, \eta)$. Ceci montre clairement que $d_{H}$ définit bien une distance sur $\partial X \backslash\{\zeta\}$.

De plus, pour tout $x \in X$, on a la formule :

$$
d_{H}\left(P_{H} \xi, P_{H} \eta\right)=e^{\frac{1}{2}\left[\beta_{\xi}\left(x, P_{H} \xi\right)+\beta_{\eta}\left(x, P_{H} \eta\right)\right]} d_{x}(\xi, \eta) .
$$

Pour deux horosphères $H$ et $H^{\prime}$ dans $X$ et $\xi$ un point de $\partial X$ autre que les bases de $H$ et $H^{\prime}$, définissons $\beta_{\xi}\left(H, H^{\prime}\right)=\beta_{\xi}\left(P_{H} \xi, P_{H^{\prime}} \xi\right)$, avec cet abus de notation qui aura l'avantage de faire apparaître l'analogie formelle entre la propriété de conformité de la famille $\left\{d_{x}\right\}_{x \in X}$ et celle de $\left\{d_{H}\right\}_{H \in \mathcal{H}}$ qui se présente ainsi :

$$
d_{H^{\prime}}\left(P_{H^{\prime}} \xi, P_{H^{\prime}} \eta\right)=e^{\frac{1}{2}\left[\beta_{\xi}\left(H, H^{\prime}\right)+\beta_{\eta}\left(H, H^{\prime}\right)\right]} d_{H}\left(P_{H} \xi, P_{H} \eta\right)
$$

À noter aussi, dans le même ordre d'idées, l'invariance par isométrie de la famille $\left\{d_{H}\right\}$ :

$$
d_{\phi H}\left(P_{\phi H} \phi \xi, P_{\phi H} \phi \eta\right)=d_{H}\left(P_{H} \xi, P_{H} \eta\right) .
$$

Signalons que la distance sur $H$ correspondant à la métrique induite par celle de $X$ (dans le langage des variétés riemanniennes) ne coïncide avec $d_{H}$ que si $X$ est un espace hyperbolique (courbure constante).

Soit maintenant $\mu$ une densité conforme de dimension $\delta$. Nous lui associerons la famille de mesures $\left\{\mu_{H}\right\}_{H \in \mathcal{H}}$, où chaque $\mu_{H}$ est définie sur $H$, plus précisément sur 
l'image de $P_{H}$, par

$$
d\left(P_{H}^{*} \mu_{H}\right)(\xi)=d \mu_{H}\left(P_{H} \xi\right)=e^{\delta \beta_{\xi}\left(x, P_{H} \xi\right)} d \mu_{x}(\xi),
$$

cette formule étant indépendante du choix de $x \in X$. La conformité de dimension $\delta$ de la densité $\mu$ se projette sur la famille $\left\{\mu_{H}\right\}_{H \in \mathcal{H}}$ suivant une analogie formelle :

$$
\frac{d P_{H^{\prime}}^{*} \mu_{H^{\prime}}}{d P_{H}^{*} \mu_{H}}(\xi)=e^{-\delta \beta_{\xi}\left(H^{\prime}, H\right)} .
$$

Si de plus $\mu$ est invariante par $\Gamma$, alors $\gamma_{*} \mu_{H}=\mu_{\gamma H}$ pour tout $\gamma \in \Gamma$ et tout $H \in \mathcal{H}$.

Tous les objets précédemment définis sur les images des $P_{H}(H \in \mathcal{H})$ se transportent sur les horosphères stables et instables dans $S X$. En effet, étant donné $u \in$ $S X$, en appelant $H$ l'horosphère dans $X$ contenant les points de base des éléments de $H^{-}(u)$ (resp. $H^{+}(u)$ ), il est clair que $H^{-}(u)$ (resp. $H^{+}(u)$ ) correspond exactement à l'image de $P_{H}$ par la projection canonique de $S X$ dans $X$; nous ferons ainsi correspondre $P_{H^{ \pm}(u)}$ à $P_{H}, d_{H^{ \pm}(u)}$ à $d_{H}$, etc. Notons que la projection

$$
P_{H^{ \pm}(u)}: \partial X \backslash\left\{g^{\mp \infty} u\right\} \longrightarrow H^{ \pm}(u)
$$

est maintenant bijective, et que $d_{H^{ \pm}(u)}$ est une distance sur $H^{ \pm}(u)$. La boule ouverte stable (resp. instable) de centre $u$ et de rayon $r$ pour la distance considérée sera notée $B^{-}(u, r)$ (resp. $\left.B^{+}(u, r)\right)$.

Il est clair que pour tout $u \in S X, \xi \in \partial X \backslash\left\{g^{\mp \infty} u\right\}$ et $t \in \mathbb{R}$, on a

$$
\beta_{\xi}\left(H^{ \pm}(u), H^{ \pm}\left(g^{t} u\right)\right)= \pm t .
$$

En particulier, on a :

$$
g^{t} B^{ \pm}(u, r)=B^{ \pm}\left(g^{t} u, e^{ \pm t} r\right)
$$

et

$$
\left(g^{t}\right)_{*} \mu_{H^{ \pm}\left(g^{t} u\right)}=e^{ \pm \delta t} \mu_{H^{ \pm}(u)}
$$

pour une densité $\mu$ conforme de dimension $\delta$. Notons aussi que

$$
\mathcal{I} B^{+}(u, r)=B^{-}(\mathcal{I} u, r) \quad \text { et } \quad \mathcal{I}_{*} \mu_{H^{+}(u)}=\mu_{H^{-}(\mathcal{I} u)} .
$$

La proposition suivante décrit la désintégration d'une mesure de BMS (généralisée) suivant les trois feuilletages dont $S X$ est en quelque sorte la somme, à savoir les feuilletages stable et instable, auxquels on adjoint les lignes du flot géodésique. La structure mise à jour est souvent appelée « produit local ». On constate également que les mesures $\mu_{H^{ \pm}(u)}$ apparaissent comme les mesures conditionnelles des mesures de BMS suivant le feuilletage (in)stable - mais il était bien plus simple et plus naturel de les introduire comme ci-dessus. 
Proposition 1.12. - Soient $\nu$ et $\mu$ deux densités conformes de dimensions respectives $\delta_{\nu}$ et $\delta_{\mu}$. Soit $u_{0} \in S X$ tel que $\nu_{o}\left(\left\{g^{+\infty} u_{0}\right\}\right)=0$. Pour toute fonction $h$ borélienne positive sur $S X$, on a

$$
\begin{aligned}
& \text { (1) } \int_{S X} \varphi(u) d m^{\nu, \mu}(u)=\int_{\mathbb{R}} d t \int_{H^{-}\left(g^{t} u_{0}\right)} d \nu_{H^{-}\left(g^{t} u_{0}\right)}\left(u_{1}\right) \int_{H^{+}\left(u_{1}\right)} d \mu_{H^{+}\left(u_{1}\right)}(u) \varphi(u) . \\
& \quad \int_{S X} \varphi(u) d m^{\nu, \mu}(u) \\
& =\int_{H^{-}\left(u_{0}\right)} d \nu_{H^{-}\left(u_{0}\right)}\left(u_{1}\right) \int_{H^{+}\left(u_{1}\right)} d \mu_{H^{+}\left(u_{1}\right)}\left(u_{2}\right) \int_{\mathbb{R}} d t e^{\left(\delta_{\mu}-\delta_{\nu}\right) t} \varphi\left(g^{t} u_{2}\right) .
\end{aligned}
$$

Démonstration. — Dans le membre de droite de (2) ci-dessus, on écrit

$$
u=g^{t} u_{2}=(\xi, \eta, s) \in \partial^{2} X \times \mathbb{R}=S X ;
$$

dans ces conditions, $\xi$ décrit $\partial X \backslash\left\{g^{-\infty} u_{0}\right\}$, et l'on a

$$
\begin{gathered}
\xi=g^{-\infty} u_{1}, \quad \eta=g^{+\infty} u_{2}, \quad d \nu_{H^{-}\left(u_{0}\right)}\left(u_{1}\right)=e^{\delta_{\nu} \beta_{\xi}\left(x, u_{1}\right)} d \nu_{x}(\xi), \\
d \mu_{H^{+}\left(u_{1}\right)}\left(u_{2}\right)=e^{\delta_{\mu} \beta_{\eta}\left(x, u_{2}\right)} d \mu_{x}(\eta)
\end{gathered}
$$

et

$$
t=\beta_{\eta}\left(u_{2}, u\right)=-\beta_{\xi}\left(u, u_{2}\right)=-\beta_{\xi}\left(u, u_{1}\right), \quad d t=d s .
$$

Il en ressort l'identité (2), d'après la définition même de $m^{\nu, \mu}$, compte tenu du fait que $\nu_{x}\left(\left\{g^{+\infty} u_{0}\right\}\right)=0$. On tire facilement (1) de (2), avec le théorème de Fubini.

\section{H. Quelques lemmes techniques}

Eu égard à la structure de produit local présentée ci-dessus, il nous sera utile d'introduire des espèces de pavés formés par les trois feuilletages fondamentaux de $S X$ liés au flot géodésique. Nous les appellerons cellules à l'instar de $[\mathbf{R u}]$, et les définissons comme suit : pour $u \in S X, E^{-} \subset H^{-}(u), E^{+} \subset H^{+}(u)$ et $r>0$, on notera

$$
C\left(E^{-}, E^{+}, r\right)=\bigcup_{|s|<r} g^{s} \bigcup_{v \in E^{-}} P_{H^{+}(v)} P_{H^{+}(u)}^{-1}\left(E^{+}\right) .
$$

En particulier, nous ferons usage de la cellule de centre $u \in S X$ et de côtés $r_{1}, r_{2}, r_{3}>0$ définie par

$$
C\left(u ; r_{1}, r_{2}, r_{3}\right)=C\left(B^{-}\left(u, r_{1}\right), B^{+}\left(u, r_{2}\right), r_{3}\right) .
$$

Ces cellules de centre $u$ forment une base de voisinages ouverts de $u$ dans $S X$. Notons que

$$
g^{t} C\left(u ; r_{1}, r_{2}, r_{3}\right)=C\left(g^{t} u ; e^{-t} r_{1}, e^{t} r_{2}, r_{3}\right) .
$$

Le lemme technique suivant et son corollaire ne sont là qu'en vue d'établir les lemmes 1.15 et 1.16 dont nous nous servirons par la suite. 
LEMME 1.13. - Soit $\varepsilon>0$. Si $r>0$ est assez petit, alors pour tout $v \in B^{-}(u, r)$ et tout $w \in B^{+}(u, 2)$, on $a$

$$
P_{H^{+}(v)} P_{H^{+}(u)}^{-1} w \in \bigcup_{|s|<\varepsilon} g^{s} B^{-}(w, \varepsilon) .
$$

En particulier, l'on a

$$
\left|\beta_{g^{+\infty}}\left(H^{+}(u), H^{+}(v)\right)\right|<\varepsilon .
$$

Démonstration. - Par souci d'allégement, nous noterons momentanément $u^{ \pm}=$ $g^{ \pm \infty} u$, etc.

Soit

Introduisons

$$
v^{\prime}=P_{H^{+}(v)} P_{H^{+}(u)}^{-1} w=P_{H^{+}(v)} w^{+} .
$$

$$
w^{\prime}=P_{H^{-}(w)} P_{H^{-}(u)}^{-1} v=P_{H^{-}(w)} v^{-} .
$$

On voit que $v^{\prime}=g^{s} w^{\prime}$ pour un $s \in \mathbb{R}$. Il nous reste à majorer $|s|$ et $d_{H^{-}(w)}\left(w^{\prime}, w\right)$. Quant à la dernière affirmation de l'énoncé, elle découlera de la première, puisque

$$
\beta_{g^{+\infty} w}\left(H^{+}(u), H^{+}(v)\right)=\beta_{w^{+}}\left(w, v^{\prime}\right)=\beta_{w^{+}}\left(w^{\prime}, v^{\prime}\right)=s .
$$

Justifions d'abord la formule suivante qui donne $s$ :

$$
e^{\frac{1}{2} s}=\frac{d_{u}\left(w^{+}, u^{-}\right)}{d_{u}\left(w^{+}, v^{-}\right)} d_{u}\left(u^{+}, v^{-}\right) .
$$

En usant de la propriété de conformité des distances visuelles, on a

$$
d_{u}\left(u^{+}, v^{-}\right)=e^{\frac{1}{2} \beta_{v^{-}}(v, u)} d_{v}\left(u^{+}, v^{-}\right)=e^{\frac{1}{2} \beta_{v^{-}}(v, u)}
$$

car $u^{+}=v^{+}$, de même

$$
d_{u}\left(w^{+}, u^{-}\right)=e^{\frac{1}{2} \beta_{w+}(w, u)}
$$

$\left(\operatorname{car} u^{-}=w^{-}\right)$, enfin

$$
d_{u}\left(w^{+}, v^{-}\right)=e^{\frac{1}{2}\left[\beta_{v}-\left(w^{\prime}, u\right)+\beta_{w+}\left(w^{\prime}, u\right)\right]}
$$

$\left(\operatorname{car} w^{\prime+}=w^{+}\right.$et $\left.w^{\prime-}=v^{\prime}\right)$. Il vient

$$
2 \log \left(\frac{d_{u}\left(w^{+}, u^{-}\right)}{d_{u}\left(w^{+}, v^{-}\right)} d_{u}\left(u^{+}, v^{-}\right)\right)=\beta_{v^{-}}\left(v, w^{\prime}\right)+\beta_{w^{+}}\left(w, w^{\prime}\right)=\beta_{v^{-}}\left(v^{\prime}, w^{\prime}\right)=s,
$$

d'où la formule annoncée.

En se servant de la relation entre distances sur les horosphères et distances visuelles (voir plus haut), on vérifie que

$$
r>d_{H^{-}(u)}(u, v)=e^{\frac{1}{2} \beta_{v^{-}}(u, v)} d_{u}\left(u^{-}, v^{-}\right)
$$

et

$$
2>d_{H^{+}(u)}(u, w)=e^{\frac{1}{2} \beta_{w^{+}}(u, w)} d_{u}\left(u^{+}, w^{+}\right) .
$$

On obtient, en joignant à cela deux formules vues auparavant, que

$$
d_{u}\left(u^{-}, v^{-}\right)<r d_{u}\left(u^{+}, v^{-}\right) \quad \text { et } \quad d_{u}\left(u^{+}, w^{+}\right)<2 d_{u}\left(w^{+}, u^{-}\right) .
$$


Avec l'inégalité triangulaire, on en déduit que

$$
\begin{aligned}
& \left|d_{u}\left(w^{+}, u^{-}\right)-d_{u}\left(w^{+}, v^{-}\right)\right| \leqslant d_{u}\left(u^{-}, v^{-}\right)<r d_{u}\left(u^{+}, v^{-}\right) \\
& \leqslant r\left[d_{u}\left(u^{+}, w^{+}\right)+d_{u}\left(w^{+}, v^{-}\right)\right]<4 r \frac{1}{2}\left[d_{u}\left(w^{+}, u^{-}\right)+d_{u}\left(w^{+}, v^{-}\right)\right],
\end{aligned}
$$

et que

$$
\left|d_{u}\left(u^{+}, v^{-}\right)-1\right| \leqslant d_{u}\left(u^{-}, v^{-}\right)<r
$$

(on a $1=d_{u}\left(u^{+}, u^{-}\right)$). Pourvu que $r$ soit assez petit, on aboutit à la conclusion désirée, à savoir que $|s|<\varepsilon$. Pour finir, on vérifie, grâce à la conformité de la famille des distances sur les horosphères, que

$$
d_{H^{-}(w)}\left(w^{\prime}, w\right)=e^{\frac{1}{2} \beta_{v^{-}}\left(v, w^{\prime}\right)} d_{H^{-}(u)}(u, v)<e^{\frac{1}{2} s} r<\varepsilon,
$$

quitte à réduire $r$.

Corollaire 1.14. - Soit $\varepsilon>0$. Si $r_{1}, r_{2}, r_{3}>0$ sont assez petits, alors pour tout $u \in S X$ et pour tout $v \in C\left(u ; r_{1}, r_{2}, r_{3}\right)$, on a

$$
B^{+}\left(v, e^{-\varepsilon}\right) \subset P_{H^{+}(v)} P_{H^{+}(u)}^{-1}\left(B^{+}(u, 1)\right) \subset B^{+}\left(v, e^{\varepsilon}\right) .
$$

Démonstration. - D'après la propriété de conformité des distances sur les horosphères (voir supra), on voit, pour $v \in H^{-}(u)$ et $w \in H^{+}(u)$, que

$$
d_{H^{+}(v)}\left(P_{H^{+}(v)} P_{H^{+}(u)}^{-1} w, v\right)=e^{\frac{1}{2} \beta_{g^{+\infty} w}\left(H^{+}(u), H^{+}(v)\right)} d_{H^{+}(u)}(w, u) .
$$

Ceci étant acquis, considérons à présent $v \in C\left(u ; r_{1}, r_{2}, r_{3}\right)$. On a $v=g^{t} v_{2}$ avec $|t|<r_{3}$ et $v_{2} \in P_{H^{+}\left(v_{1}\right)} P_{H^{+}(u)}^{-1}\left(B^{+}\left(u, r_{2}\right)\right)$ où $v_{1} \in B^{-}\left(u, r_{1}\right)$. Grâce à ce qui précède, compte tenu de la dernière affirmation du lemme 1.13 (où l'on mettra $\varepsilon / 3$ en place de $\varepsilon)$, on a, pourvu que $r_{1}$ soit assez petit :

$$
B^{+}\left(v_{1}, e^{-\varepsilon / 3}\right) \subset P_{H^{+}\left(v_{1}\right)} P_{H^{+}(u)}^{-1}\left(B^{+}(u, 1)\right) \subset B^{+}\left(v_{1}, e^{\varepsilon / 3}\right),
$$

et d'autre part

$\left(\right.$ si $\left.r_{2}<2\right)$ puisque

$$
d_{H^{+}\left(v_{1}\right)}\left(v_{2}, v_{1}\right)<e^{\varepsilon / 3} r_{2}
$$

$$
v_{2} \in P_{H^{+}\left(v_{1}\right)} P_{H^{+}(u)}^{-1}\left(B^{+}\left(u, r_{2}\right)\right)
$$

avec $v_{1} \in B^{-}\left(u, r_{1}\right)$. Il en résulte que

$$
B^{+}\left(v_{2}, e^{-2 \varepsilon / 3}\right) \subset P_{H^{+}\left(v_{2}\right)} P_{H^{+}(u)}^{-1}\left(B^{+}(u, 1)\right) \subset B^{+}\left(v_{2}, e^{2 \varepsilon / 3}\right),
$$

pourvu que $r_{1}$ et $r_{2}$ soient assez petits. Pour $r_{3}<\varepsilon / 3$, la conclusion désirée s'ensuit en composant par $g^{t}$.

Le lemme suivant précise le conditionnement d'une mesure de BMS sur les horosphères instables (un énoncé analogue vaut bien sûr pour les horosphères stables). Il signifie que la moyenne de l'indicatrice d'un borélien quelconque sur n'importe quelle cellule assez petite est arbitrairement proche de la moyenne sur le côté instable de cette cellule, pourvu toutefois que l'on épaississe ou rétrécisse un peu le borélien en 
question selon les directions stable et géodésique, mais non selon l'instable, ce qui se révélera fondamental dans les applications que nous en ferons.

Introduisons une notation pour les altérations d'un borélien évoquées à l'instant. Pour $u \in S X$ et $\varepsilon>0$, nous désignerons par $u_{\varepsilon}$ l'ensemble $\bigcup_{|s|<\varepsilon} g^{s} B^{-}(u, \varepsilon)$; ensuite, pour $A \subset S X$, nous noterons $A_{\varepsilon}=\bigcup_{a \in A} a_{\varepsilon}$ et $A_{-\varepsilon}=\left\{a \in A \mid a_{\varepsilon} \subset A\right\}$. On vérifie aisément que pour $t \geqslant 0$, l'on a $\left[g^{-t} A\right]_{\varepsilon} \subset g^{-t} A_{\varepsilon}$, ainsi que $\left[g^{-t} A\right]_{-\varepsilon} \supset g^{-t} A_{-\varepsilon}$. Cette propriété paraîtra essentielle puisque l'on appliquera par la suite le lemme 1.15 à toute la famille $\operatorname{des} g^{-t} A(t \geqslant 0)$ simultanément.

Lemme 1.15. - Soient $\nu$ et $\mu$ deux densités conformes non atomiques. Soit $\varepsilon>0$. Si $r_{1}, r_{3}>0$ sont suffisamment petits, alors, pour tout $u \in S X$, pour tous boréliens

$$
E^{-} \subset B^{-}\left(u, r_{1}\right) \text { et } E^{+} \subset B^{+}(u, 1),
$$

et pour tout borélien $A \subset S X$, on a l'encadrement :

et

$$
\begin{aligned}
2 r_{3} \nu_{H^{-}(u)}\left(E^{-}\right) \mu_{H^{+}(u)}\left(E^{+} \cap A\right) & \leqslant e^{\varepsilon} m^{\nu, \mu}\left(C\left(E^{-}, E^{+}, r_{3}\right) \cap A_{\varepsilon}\right) \\
m^{\nu, \mu}\left(C\left(E^{-}, E^{+}, r_{3}\right) \cap A\right) & \leqslant e^{\varepsilon} 2 r_{3} \nu_{H^{-}(u)}\left(E^{-}\right) \mu_{H^{+}(u)}\left(E^{+} \cap A_{\varepsilon}\right) .
\end{aligned}
$$

Pour obtenir des encadrements à proprement parler, on utilisera que $\left[A_{-\varepsilon}\right]_{\varepsilon} \subset A$.

D'autre part, l'on préférera parfois l'énoncé équivalent à ce dernier obtenu en y remplaçant l'indicatrice de $A$ par une quelconque fonction borélienne positive $h$; à ce propos, précisons les notations $h_{\varepsilon}(u)=\sup _{v \in u_{\varepsilon}} h(v)$ et $h_{-\varepsilon}(u)=\inf _{v \in u_{\varepsilon}} h(v)$ (on a bien sûr $\left[\mathbb{1}_{A}\right]_{ \pm \varepsilon}=\mathbb{1}_{A_{ \pm \varepsilon}}$.

Démonstration. - Soit $h$ une fonction borélienne positive sur $S X$ (en particulier $\mathbb{1}_{A}$ ). En reprenant la définition des cellules et en appliquant la proposition 1.12 (avec $u_{0}=u$ ), on obtient que

$$
\begin{aligned}
\int_{C\left(E^{-}, E^{+}, r_{3}\right)} & h d m^{\nu, \mu} \\
= & \int_{E^{-}} d \nu_{H^{-}(u)}(v) \int_{P_{H^{+}(v)} P_{H^{+}(u)}^{-1}\left(E^{+}\right)} d \mu_{H^{+}(v)}(w) \int_{-r_{3}}^{r_{3}} d t e^{\left(\delta_{\mu}-\delta_{\nu}\right) t} h\left(g^{t} w\right) .
\end{aligned}
$$

Or

$$
\int_{-r_{3}}^{r_{3}} d t e^{\left(\delta_{\mu}-\delta_{\nu}\right) t} h\left(g^{t} w\right) \leqslant e^{\varepsilon / 2} 2 r_{3} h_{\varepsilon / 2}(w)
$$

pourvu que $r_{3}$ soit assez petit. De plus, grâce à la conformité des mesures sur les horosphères, on a

$$
\begin{aligned}
& \int_{P_{H^{+}(v)} P_{H^{+}(u)}^{-1}\left(E^{+}\right)} d \mu_{H^{+}(v)}(w) h_{\varepsilon / 2}(w)
\end{aligned}
$$

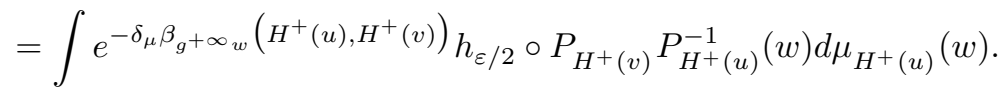

MÉMOIRES DE LA SMF 95 
En s'appuyant sur le lemme 1.13, on en déduit la seconde inégalité de l'énoncé, avec $r_{1}, r_{3}$ assez petits (indépendamment de $u$ et de $h$ ).

Inversement, en considérant $v \in E^{-} \subset B^{-}\left(u, r_{1}\right)$, on a

$$
\begin{aligned}
& \int_{E^{+}} h d \mu_{H^{+}(u)} \\
& \quad=\int_{P_{H^{+}(v)} P_{H^{+}(u)}^{-1}\left(E^{+}\right)} e^{-\delta_{\mu} \beta_{g^{+\infty}}\left(H^{+}(v), H^{+}(u)\right)} h \circ P_{H^{+}(u)} P_{H^{+}(v)}^{-1}(w) d \mu_{H^{+}(v)}(w) .
\end{aligned}
$$

Or

$$
u \in B^{-}\left(v, r_{1}\right) \quad \text { et } \quad P_{H^{+}(v)} P_{H^{+}(u)}^{-1}\left(E^{+}\right) \subset P_{H^{+}(v)} P_{H^{+}(u)}^{-1}\left(B^{+}\left(u, r_{1}\right)\right) \subset B^{+}(v, 2)
$$

d'après le corollaire 1.14, pourvu que $r_{1}$ soit assez petit. En utilisant alors le lemme 1.13 , on en déduit que

$$
\int_{E^{+}} h d \mu_{H^{+}(u)} \leqslant e^{\varepsilon / 2} \int_{P_{H^{+}(v)} P_{H^{+}(u)}^{-1}\left(E^{+}\right)} d \mu_{H^{+}(v)}(w) h_{\varepsilon / 2}(w)
$$

(si $r_{1}$ est assez petit). Avec le fait que

$$
2 r_{3} h_{\varepsilon / 2}(w) \leqslant e^{\varepsilon / 2} \int_{-r_{3}}^{r_{3}} d t e^{\left(\delta_{\mu}-\delta_{\nu}\right) t} h_{\varepsilon}\left(g^{t} w\right)
$$

dès que $r_{3}$ est assez petit, on obtient en définitive la première inégalité de l'énoncé.

Un dernier lemme nous sera utile dans le chapitre 6 (proposition 6.2 et théorème 6.4). Il montre que les moyennes sur les boules instables jouissent d'une certaine propriété de continuité.

Lemme 1.16. - Soit $\mu$ une densité conforme non atomique. Soient $\varepsilon>0$ et $r>0$. Si $r_{1}, r_{2}, r_{3}>0$ sont suffisamment petits, alors pour tout $u \in S X$, pour tout $v \in$ $C\left(E^{-}, E^{+}, r_{3}\right)$ et pour tout borélien $A \subset S X$, on a l'encadrement :

$$
\begin{aligned}
e^{-\varepsilon} \mu_{H^{+}(u)}\left(B^{+}\left(u, r e^{-\varepsilon}\right) \cap A_{-\varepsilon}\right) \leqslant \mu_{H^{+}(v)}\left(B^{+}(v, r)\right. & \cap A) \\
& \leqslant e^{\varepsilon} \mu_{H^{+}(u)}\left(B^{+}\left(u, r e^{\varepsilon}\right) \cap A_{\varepsilon}\right) .
\end{aligned}
$$

Les mêmes remarques que celles qui suivaient le lemme 1.15 restent de mise ici.

Démonstration. - On peut supposer $0<\varepsilon<\log 2$. On commence par se ramener au cas $r=1$ en composant par $g^{-\log r}$. Soit $h$ une fonction borélienne positive sur $S X$. Considérons $v \in C\left(u ; r_{1}, r_{2}, r_{3}\right)$. D'après la propriété de conformité de la famille $\left\{\mu_{H^{+}}\right\}$, on a

$$
\begin{aligned}
& \int_{P_{H^{+}(v)} P_{H^{+}(u)}^{-1}\left(B^{+}\left(u, e^{ \pm \varepsilon}\right)\right)} h d \mu_{H^{+}(v)} \\
& =\int_{B^{+}\left(u, e^{ \pm \varepsilon}\right)} h \circ P_{H^{+}(v)} P_{H^{+}(u)}^{-1}(w) e^{-\delta_{\mu} \beta_{g^{+} \infty}\left(H^{+}(u), H^{+}(v)\right)} d \mu_{H^{+}(u)}(w) .
\end{aligned}
$$


On a $v=g^{s} v_{2}$ avec $|s|<r_{3}$ et $v_{2} \in P_{H^{+}\left(v_{1}\right)} P_{H^{+}(u)}^{-1}\left(B^{+}\left(u, r_{2}\right)\right)$ où $v_{1} \in B^{-}\left(u, r_{1}\right)$. Pour $w \in B^{+}\left(u, e^{ \pm}\right) \subset B^{+}(u, 2)$, on voit, en utilisant le lemme 1.13, que

$$
P_{H^{+}\left(v_{1}\right)} P_{H^{+}(u)}^{-1} w=g^{s} P_{H^{+}\left(v_{1}\right)} P_{H^{+}(u)}^{-1} w \in w_{\varepsilon},
$$

ainsi que

$$
\left|\beta_{g^{+\infty} w}\left(H^{+}(u), H^{+}(v)\right)\right| \leqslant \varepsilon / \delta_{\mu},
$$

à condition que $r_{1}$ et $r_{3}$ soient assez petits. En outre, le corollaire 1.14, légèrement modifié au moyen de compositions par $g^{ \pm \log \varepsilon}$, nous donne :

$$
P_{H^{+}(v)} P_{H^{+}(u)}^{-1}\left(B^{+}\left(u, e^{-\varepsilon}\right)\right) \subset B^{+}(v, 1) \subset P_{H^{+}(v)} P_{H^{+}(u)}^{-1}\left(B^{+}\left(u, e^{\varepsilon}\right)\right)
$$

pourvu que $r_{1}, r_{2}, r_{3}$ soient assez petits. Tous ces faits réunis emportent la conclusion. 


\section{CHAPITRE 2}

\section{ERGODICITÉ DU FEUILLETAGE HOROSPHÉRIQUE}

Pour traiter de l'ergodicité du feuilletage horosphérique, nous reprendrons une notion introduite par K. Schmidt dans [Sch], à savoir celle de valeur essentielle pour $\Gamma$ par rapport au cocycle $\beta$ de Busemann — inséparable des horosphères. Un réel $t \in \mathbb{R}$ sera appelé valeur essentielle si pour tout borélien $B$ de $\partial X$ de $\mu_{o}$-mesure non nulle et pour tout $\varepsilon>0$, il existe $\gamma \in \Gamma$ tel que l'ensemble $B \cap \gamma B \cap\left\{\xi|| \beta_{\xi}\left(o, \gamma_{o}\right)-t \mid<\varepsilon\right\}$ ne soit pas de $\mu_{o}$-mesure nulle. Il est aisé de se rendre compte que l'ensemble $\mathcal{E}$ de ces valeurs essentielles forme un sous-groupe fermé de $\mathbb{R}$ (on pourra consulter [Sch]). Il convient de faire observer ici que $\mathcal{E}$ ne dépend point du choix de $o$ dans $X$, et qu'il est ainsi loisible de remplacer $o$ par un quelconque point $x \in X$ dans l'énoncé de la définition précédente (en effet, comme $\xi \mapsto \beta_{\xi}(o, x)$ est continue, on peut restreindre $B$ à un borélien où cette fonction varie arbitrairement peu). L'intérêt de la notion de valeur essentielle réside dans la caractérisation suivante de l'ergodicité du feuilletage horosphérique. Cet énoncé est un cas particulier d'un théorème général de K. Schmidt $([\mathbf{S c h}])$, mais nous en redonnons une preuve directe par souci de complémentation. L'idée sous-jacente consiste très simplement à observer qu'une fonction invariante par le feuilletage horosphérique sera également invariante par le flot géodésique pourvu que $\mathcal{E}$ épuise $\mathbb{R}$, et qu'elle induira alors une fonction invariante sur $\partial X$.

Proposition 2.1 (Schmidt). - Soit $\mu$ une densité conforme invariante par $\Gamma$. Alors $(\mathcal{H}, \Gamma, \widehat{\mu})$ est ergodique si et seulement si $\left(\partial X, \Gamma, \mu_{o}\right)$ est ergodique et $\mathcal{E}=\mathbb{R}$.

Démonstration. - Supposons l'ergodicité de $(\mathcal{H}, \Gamma, \widehat{\mu})$. Celle de $\left(\partial X, \Gamma, \mu_{o}\right)$ est immédiate, $\mathcal{H}$ étant fibré au-dessus de $\partial X$. Prenons maintenant $t \in \mathbb{R}$ quelconque, et prouvons que $t \in \mathcal{E}$. Étant donnés $\varepsilon>0$ et un borélien $B$ de $\partial X$ tel que $\mu_{o}(B)>0$, considérons, pour $s \in \mathbb{R}$, l'ensemble $\mathcal{B}_{s}$ des horosphères de base $\xi$ dans $B$ et passant par un point $x$ tel que $\left|\beta_{\xi}(o, x)-s\right|<\varepsilon / 2$. Puisque $\widehat{\mu}\left(\mathcal{B}_{0}\right) \geqslant \mu_{o}(B) \varepsilon e^{-\varepsilon / 2}>0$, nécessairement $\widehat{\mu}\left(\mathcal{H} \backslash \Gamma \mathcal{B}_{0}\right)=0$ par l'ergodicité de $(\mathcal{H}, \Gamma, \widehat{\mu})$. Il existe donc $\gamma \in \Gamma$ tel que $\widehat{\mu}\left(\mathcal{B}_{t} \cap \gamma \mathcal{B}_{0}\right)>0$. Mais si $\xi$ est la base d'une horosphère de $\mathcal{B}_{t} \cap \gamma \mathcal{B}_{0}$, on a $\xi \in B$, 
$\gamma^{-1} \xi \in B$, et, $x$ désignant un point de ladite horosphère dans $X$,

$$
\left|\beta_{\xi}(o, \gamma o)-t\right| \leqslant\left|\beta_{\xi}(o, x)-t\right|+\left|\beta_{\xi}(x, \gamma o)\right|<\varepsilon .
$$

On en conclut que

$$
B \cap \gamma B \cap\left\{\xi|| \beta_{\xi}(o, \gamma o)-t \mid<\varepsilon\right\}
$$

a une $\mu_{o}$-mesure non nulle. Ainsi $t \in \mathcal{E}$.

Réciproquement, supposons que $\left(\partial X, \Gamma, \mu_{o}\right)$ soit ergodique et que $\mathcal{E}=\mathbb{R}$. Soit $h$ une fonction de $\mathcal{H}$ dans $[0,1]$, borélienne et invariante par $\Gamma$; montrons que $h$ est $\widehat{\mu}$-essentiellement constante. Il s'avérera commode d'avoir régularisé $h$ le long de la composante réelle de $\mathcal{H}=\partial X \times \mathbb{R}$, en considérant les fonctions $h_{\tau}(\tau>0)$ définies par

$$
h_{\tau}(H)=\int_{0}^{\tau} h\left(g^{t} H\right) d t \quad \text { pour } H \in \mathcal{H}
$$

où le flot $g^{t}$ est défini par $g^{t}(\xi, s)=(\xi, s+t)$ pour $(\xi, s) \in \mathcal{H}$ (ce flot étant une projection sur $\mathcal{H}$ du flot géodésique sur $S X$, nous nous sommes permis un abus de notation). Nous allons montrer ci-après que pour tout $\tau>0$, la fonction $h_{\tau}$ (encore invariante par $\Gamma$ ) est invariante par $g^{t}$, pour tout $t \in \mathbb{R}$. Nous aurons alors que $h_{\tau} \circ g^{t}=h_{\tau}$ pour tout $t \in \mathbb{R}$, ce $\widehat{\mu}$-p.p, compte tenu de la continuité de $h_{\tau}$ le long des lignes du flot $g^{t}$. Ainsi $h_{\tau}$ apparaîtra comme étant le relevé d'une fonction de $\partial X$ dans $\mathbb{R}$ invariante par $\Gamma$, et de ce fait $\mu_{o}$-essentiellement constante par l'ergodicité de $\left(\partial X, \Gamma, \mu_{o}\right)$. Ainsi aurons-nous que $h_{\tau}$ est égale $\widehat{\mu}$-p.p à une fonction constante $c(\tau)$. De la relation

$$
h_{\tau+\sigma}=h_{\tau}+h_{\sigma} \circ g^{\tau},
$$

il vient que $c$ est linéaire, c'est-à-dire de la forme $c(\tau)=\kappa \tau$. Compte tenu du fait que $\left|h_{\tau+\sigma}-h_{\tau}\right| \leqslant \sigma$, on en déduit (avec un argument de densité dénombrable dans $\mathbb{R}$ ) que $\widehat{\mu}$-p.p, on a l'égalité $h_{\tau}=\kappa \tau$ pour tout $\tau>0$. Or, en recourant à un théorème classique, on sait que $\frac{1}{\tau} h_{\tau} \rightarrow \varphi$ en $\widehat{\mu}$-presque tout point quand $\tau \rightarrow 0$. Par suite, $h=\kappa(\widehat{\mu}$-p.p), ce qu'il fallait montrer.

Reste à prouver - et c'est là le cœur de la démonstration - que pour un $t \in \mathbb{R}$ fixé, on a $\psi \circ g^{t}=\psi$ ( $\widehat{\mu}$-p.p), où l'on note $\psi=h_{\tau}$ pour $\tau>0$ fixé, afin d'alléger. Nous le ferons par l'absurde, en supposant que l'ensemble $\left\{\psi \circ g^{t} \neq \psi\right\}$ n'est pas de $\widehat{\mu}$-mesure nulle. Quitte à changer $\psi$ en $-\psi$, on peut supposer que l'ensemble $\left\{\psi \circ g^{t}>\psi\right\}$ a une $\widehat{\mu}$-mesure non nulle. On peut ensuite trouver $a \in \mathbb{R}$ et $\varepsilon>0$ tels que l'ensemble

$$
\mathcal{B}_{0}=\left\{\psi<a-4 \varepsilon<a+4 \varepsilon<\psi \circ g^{t}\right\}
$$

ne soit pas de $\widehat{\mu}$-mesure nulle. Choisissons un intervalle $I$ de longueur $2 \varepsilon$ tel que

$$
\widehat{\mu}\left((\partial X \times I) \cap \mathcal{B}_{0}\right)>0 .
$$

Appelons $B$ l'ensemble des $\xi \in \partial X$ tels que $(\{\xi\} \times I) \cap \mathcal{B}_{0}$ ne soit pas vide, puis $\mathcal{B}=B \times I$. Or on peut aisément vérifier que $\left|\psi \circ g^{s}-\psi\right| \leqslant 2|s|$ pour $s \in \mathbb{R}$ (on rappelle que $0 \leqslant \varphi \leqslant 1$ ). Par conséquent, pour tout $H \in \mathcal{B}$, on a $\psi(H)<a-4 \varepsilon+2 \times 2 \varepsilon=a$, 
ainsi que $\psi\left(g^{t} H\right)>a$, puisque $g^{s} H \in \mathcal{B}_{0}$ pour un $\left.s \in\right]-2 \varepsilon, 2 \varepsilon[, I$ étant de longueur $2 \varepsilon$. Or $t$ est une valeur essentielle $(\mathcal{E}=\mathbb{R})$, et $\mu_{o}(B)>0$, aussi sait-on que pour un certain $\gamma \in \Gamma$, l'ensemble

$$
A=B \cap \gamma B \cap\left\{\xi|| \beta_{\xi}(o, \gamma o)-t \mid<\varepsilon\right\}
$$

a une $\mu_{o}$-mesure non nulle. Pour $\xi \in A$, soit $I_{\xi}$ l'intervalle des $s \in I$ tels que

$$
s+\beta_{\xi}(o, \gamma o)-t \in I \text {. }
$$

Comme $I$ est de longueur $2 \varepsilon$, nécessairement $I_{\xi}$ est de longueur supérieure à $\varepsilon$, et par conséquent l'ensemble $\mathcal{A}=\bigcup_{\xi \in A}\{\xi\} \times I_{\xi}$ est de $\widehat{\mu}$-mesure non nulle. De plus, pour $H=(\xi, s) \in \mathcal{A}$, on a d'une part $\psi(H)<a$ car $H \in \mathcal{B}$; d'autre part,

$$
\gamma^{-1} H=\left(\gamma^{-1} \xi, s+\beta_{\xi}(o, \gamma o)\right)=g^{t}\left(\gamma^{-1} \xi, s+\beta_{\xi}(o, \gamma o)-t\right) \text {, }
$$

or

$$
\left(\gamma^{-1} \xi, s+\beta_{\xi}(o, \gamma o)-t\right) \in B \times I=\mathcal{B},
$$

si bien que $\psi\left(\gamma^{-1} H\right)>a$. Ainsi $\psi \circ \gamma^{-1}>\psi$ sur $\mathcal{A}$, ce qui est contradictoire avec l'invariance de $\psi$ par $\Gamma$.

Ces faits préliminaires étant acquis, nous allons maintenant établir un résultat d'ergodicité du feuilletage horosphérique sous certaines hypothèses (théorème 2.2). Bien que dans le restant de cet article, seul le corollaire 2.3 soit employé, nous présentons cependant l'énoncé général dont il découle, pour la bonne raison que la preuve de l'un ou l'autre est strictement la même (à la distinction entre $\Gamma$ et $\Gamma_{0}$ près). Qui plus est, nous comptons faire usage de ce théorème général dans une future publication traitant, on le devine, des sous-groupes distingués de certains groupes discrets d'isométries (ce qui a déjà été abordé dans [Bab-L] entre autres).

On ne s'étonnera pas de retrouver ici l'hypothèse de non-arithméticité du spectre des longueurs de $\Gamma$, dont on a appris à connaître la nécessité, puisque déjà la simple transitivité topologique du feuilletage horosphérique la requiert, et lui est même équivalente (voir théorème 1.4). Ici, cette condition provient naturellement du principe de la démonstration, qui consiste à faire apparaître les longueurs des géodésiques fermées associées à $\Gamma$ comme valeurs essentielles, selon la terminologie introduite en début de chapitre. Peut-être n'est-il pas inutile de rappeler ici que ladite condition est en fait acquise dans bien des situations a priori ( $c f$. proposition 1.6). À propos de la seconde hypothèse portant, elle, sur la densité considérée, il n'est pas besoin que $\Gamma_{0}$ soit discret - ce pourrait même être le normalisateur de $\Gamma$ dans le groupe des isométries de $X$ tout entier. Il est encore possible de remplacer $\Lambda_{M}\left(\Gamma_{0}\right)$ par des ensembles un peu plus larges, quoique au fond de même nature. On trouvera dans [Ka3] des hypothèses de nature voisine pour un résultat analogue, obtenu également par la méthode des valeurs essentielles. Le cas de la densité de Lebesgue en courbure constante et dimension deux a fait l'objet de $[\mathbf{K a 2}]$, où aucune de ces conditions n'est requise. 
THÉORÈmE 2.2. - Supposons que $\Gamma$ soit un sous-groupe distingué d'un groupe $\Gamma_{0}$ d'isométries de $X$, et que le spectre des longueurs associé à $\Gamma$ ne soit pas arithmétique. Soit $\mu$ une densité conforme et invariante par $\Gamma$, et supposons que $\mu_{o}\left(\Lambda_{M}\left(\Gamma_{0}\right)\right)=1$. Si $\left(\partial X, \Gamma, \mu_{o}\right)$ est ergodique, alors $(\mathcal{H}, \Gamma, \widehat{\mu})$ l'est également.

Du résultat ci-dessus, nous dégageons le corollaire suivant, qui constitue la pierre angulaire de tout le restant de cet article, en ouvrant directement sur le théorème 3.1 - ce dernier affirme le mélange du flot géodésique. Ce corollaire 2.3 s'obtient en appliquant le théorème 2.2 avec $\Gamma_{0}=\Gamma$, à la lumière du corollaire 1.8. L'énoncé ci-dessous signifie encore que l'ergodicité du flot géodésique (fait équivalent à la divergence du type de $\Gamma$, d'après le théorème 1.7) entraîne celle du feuilletage horosphérique (éventuellement moyennant la condition que l'on sait), relation qui a été souvent pressentie - mais qui reste grossière, la réciproque étant loin d'être vraie (on en trouvera des contre-exemples dans [Bab-L]). Signalons que le premier résultat d'ergodicité du flot horocyclique (dimension deux) remonte à [He] (cas des surfaces à courbure constante et d'aire finie), étendu aux groupes convexe-cocompacts du disque hyperbolique ayant un exposant plus grand que $1 / 2$ dans $[\mathbf{B u}]$ — où un résultat plus fort est avancé, dont nous reparlerons au chapitre 6 ; le cas des revêtements des surfaces cocompactes de fibre $\mathbb{Z}$ ou $\mathbb{Z}^{2}$, dont le type est encore divergent, avait été obtenu dans [Bab-L].

Corollaire 2.3. - Supposons que $\Gamma$ soit de type divergent et que le spectre des longueurs associé à $\Gamma$ ne soit pas arithmétique. Soit $\mu$ la densité conforme de dimension $\delta(\Gamma)$ et invariante par $\Gamma$. Alors $(\mathcal{H}, \Gamma, \widehat{\mu})$ est ergodique.

Démonstration. - Supposons que $\left(\partial X, \Gamma, \mu_{o}\right)$ soit ergodique. Ainsi la première condition du critère d'ergodicité pour $(\mathcal{H}, \Gamma, \widehat{\mu})$ de la proposition 2.1 est d'emblée satisfaite, et il nous suffit de prouver que l'ensemble $\mathcal{E}$ des valeurs essentielles est égal à $\mathbb{R}$, ce que nous obtiendrons en montrant que toute longueur de géodésique fermée associée à $\Gamma$ est dans $\mathcal{E}$, pourvu toutefois que cette longueur soit supérieure à $\log 3+1$, restriction qui bien sûr ne saurait affecter en rien que ce soit la conclusion; la non-arithméticité du spectre des longueurs fera le reste. Avant de commencer, observons une fois pour toutes que $\Lambda(\Gamma)=\Lambda\left(\Gamma_{0}\right)=\overline{\Lambda_{M}\left(\Gamma_{0}\right)}$ est précisément le support de $\mu$.

Considérons une isométrie hyperbolique $\phi \in \Gamma$ de longueur de translation $L>\log 3+1$. On notera $a \in \partial X$ son point fixe attractif. Fixons un point $b \in \Lambda(\Gamma) \backslash\{a\}$, puis un point $x \in X$ sur la géodésique $(a b)$. Étant donné $\varepsilon \in] 0,2[$ arbitrairement petit fixé, nous allons prouver que pour tout borélien $B \subset \partial X$ de $\mu_{x}$-mesure non nulle, l'ensemble

$$
B \cap \gamma B \cap\left\{\xi|| \beta_{\xi}(x, \gamma x)-L \mid<\varepsilon\right\}
$$

a une $\mu_{x}$-mesure non nulle pour au moins un $\gamma \in \Gamma$; nous aurons alors montré que $L \in \mathcal{E}$. Le principe de la preuve est le suivant. Considérons la famille $\mathcal{B}$ des $d_{x}$-boules 
de centre le point fixe attractif d'un conjugué $\gamma \phi \gamma^{-1}$ de $\phi$ où $\gamma \in \Gamma_{0}$, autrement dit de centre $\gamma a$, et de rayon $r$ assez petit pour que l'on ait

$$
\left|\beta_{\xi}\left(x, \gamma \phi \gamma^{-1} x\right)-L\right|<\varepsilon \quad \text { et }\left|\beta_{\xi}\left(x, \gamma \phi^{-1} \gamma^{-1} x\right)+L\right|<\varepsilon
$$

pour tout $\xi$ dans la boule de rayon triple $3 B_{x}(\gamma a, r)=B_{x}(\gamma a, 3 r)$ (faisons observer que $\left.\beta_{\gamma a}\left(x, \gamma \phi^{ \pm 1} \gamma^{-1} x\right)= \pm L\right)$. Les raisons des axiomes techniques précis de cette définition apparaîtront au cours de la preuve. Prenons dans $\mathcal{B}$ une boule $B_{x}(\gamma a, r)$ que nous noterons momentanément $\Delta$, et commençons par observer que pour $\xi \in \Delta$, on a

$$
\begin{aligned}
d_{x}\left(\gamma \phi \gamma^{-1} \xi, \gamma a\right) & =d_{\gamma \phi^{-1} \gamma^{-1} x}(\xi, \gamma a) \\
& =e^{\frac{1}{2}\left[\beta_{\xi}\left(x, \gamma \phi^{-1} \gamma^{-1} x\right)+\beta_{\gamma a}\left(x, \gamma \phi^{-1} \gamma^{-1} x\right)\right]} d_{x}(\xi, \gamma a)<e^{-L+\varepsilon / 2} r<r ;
\end{aligned}
$$

ainsi $\gamma \phi \gamma^{-1}(\Delta) \subset \Delta$; il s'ensuit que

$$
\Delta \cap \gamma \phi \gamma^{-1} \Delta \cap\left\{\xi|| \beta_{\xi}\left(x, \gamma \phi \gamma^{-1} x\right)-L \mid<\varepsilon\right\}=\gamma \phi \gamma^{-1} \Delta,
$$

ensemble de $\mu_{x}$-mesure non nulle. Dans le cas particulier où le borélien $B$ considéré plus haut serait une boule $\Delta$ de $\mathcal{B}$, on aurait donc la propriété désirée, puisque $\gamma \phi \gamma^{-1} \in \Gamma$. Dans le cas général, la même conclusion se maintiendrait pourvu que $B$ participât suffisamment à la $\mu_{x}$-masse d'une boule de $\mathcal{B}$. Plus précisément, supposons, pour $B$ de $\mu_{x}$-mesure non nulle, qu'il existe une boule $\Delta=B_{x}(\gamma a, r) \in \mathcal{B}$ telle que

$$
\mu_{x}(\Delta \cap B)>\left(1+e^{-\delta(L+\varepsilon)}\right)^{-1} \mu_{x}(\Delta) .
$$

Comme

$$
\mu_{x}\left(\gamma \phi \gamma^{-1}(\Delta \cap B)\right)=\int_{\Delta \cap B} e^{\delta \beta_{\xi}\left(x, \gamma \phi^{-1} \gamma^{-1} x\right)} d \mu_{x}(\xi)>e^{-\delta(L+\varepsilon)} \mu_{x}(\Delta \cap B)
$$

compte tenu du fait que $\gamma \phi \gamma^{-1} \in \Gamma$, il s'ensuivrait que

$$
\mu_{x}(\Delta \cap B)+\mu_{x}\left(\gamma \phi \gamma^{-1}(\Delta \cap B)\right)>\mu_{x}(\Delta) .
$$

Vu que $\Delta \cap B$ et $\gamma \phi \gamma^{-1}(\Delta \cap B)$ sont tous deux contenus dans $\Delta$, on en déduirait que leur intersection a une $\mu_{x}$-mesure non nulle. Enfin, comme

$$
B \cap \gamma \phi \gamma^{-1} B \cap\left\{\xi|| \beta_{\xi}\left(x, \gamma \phi \gamma^{-1} x\right)-L \mid<\varepsilon\right\} \supset \Delta \cap B \cap \gamma \phi \gamma^{-1}(\Delta \cap B),
$$

on aurait la conclusion voulue. Aussi notre objectif consiste-t-il dorénavant à prouver que l'hypothèse précédente est satisfaite, en somme que $\mathcal{B}$ est une famille dérivante pour la mesure $\mu_{x}$, ce qui signifie précisément que pour tout borélien $B$ de mesure non nulle, pour $\mu_{x}$-presque tout $\xi \in B$, on a

$$
\frac{\mu_{x}(\Delta \cap B)}{\mu_{x}(\Delta)} \longrightarrow 1
$$

lorsque le diamètre de $\Delta$, appartenant à $\mathcal{B}$ et contenant $\xi$, tend vers zéro.

Nous allons d'abord prouver qu'un quelconque point $\eta \in \Lambda_{M}\left(\Gamma_{0}\right)$ fixé appartient à une boule de $\mathcal{B}$ de $d_{x}$-rayon inférieur à un $\rho>0$ arbitrairement petit. Choisissons 
un voisinage $V$ de $b$ dans $\bar{X}$ et $r \in] 0, \rho[$ suffisamment petits pour que les conditions suivantes aient lieu :

(i) pour $z \in V \cap X$ et $\xi \in B_{x}\left(a, e^{L+\varepsilon / 4} r\right),(z, x)-\varepsilon / 2<\beta_{\xi}(z, x) \quad(\leqslant(z, x))$;

(ii) pour $\xi \in B_{x}(a, r),\left|\beta_{\xi}(x, \phi x)-L\right|<\varepsilon / 2$ et $\left|\beta_{\xi}\left(x, \phi^{-1} x\right)+L\right|<\varepsilon / 2$.

Comme $\eta \in \Lambda_{M}\left(\Gamma_{0}\right)$, nous pouvons trouver $\gamma \in \Gamma_{0}$ tel que $d_{x}\left(\gamma^{-1} \eta, a\right)<\frac{1}{3} e^{-\varepsilon / 2} r$ et $\gamma^{-1} x \in V$. Considérons alors la boule $B=B_{x}\left(\gamma a, \frac{1}{3} e^{-(x, \gamma x)} r\right)$; notons tout de suite que $B$ a un rayon inférieur à $\rho$. Nous allons maintenant montrer que $\eta \in B$ et que $B \in \mathcal{B}$. De la relation

$$
d_{x}(\gamma \xi, \gamma a)=d_{\gamma^{-1} x}(\xi, a)=e^{-\frac{1}{2}\left[\beta_{\xi}\left(\gamma^{-1} x, x\right)+\beta_{a}\left(\gamma^{-1} x, x\right)\right]} d_{x}(\xi, a),
$$

il vient d'une part que

$$
d_{x}(\gamma \xi, \gamma a) \geqslant e^{-(x, \gamma x)} d_{x}(\xi, a)
$$

pour $\xi \in \partial X$ quelconque, d'autre part que

$$
d_{x}(\gamma \xi, \gamma a)<e^{\varepsilon / 2-(x, \gamma x)} d_{x}(\xi, a)
$$

pour $\xi \in B_{x}(a, r)$ grâce à (i) ci-dessus. De là, on obtient d'une part que

$$
\gamma B_{x}(a, r) \supset B_{x}\left(\gamma a, e^{-(x, \gamma x)} r\right)=3 B
$$

(puisque $\gamma$ est un homéomorphisme et que

$$
d_{x}(\gamma \xi, \gamma a) \geqslant e^{-(x, \gamma x)} r
$$

dès que $\left.d_{x}(\xi, a) \geqslant r\right)$, d'autre part que

$$
\gamma B_{x}\left(a, \frac{1}{3} e^{-\varepsilon / 2} r\right) \subset B .
$$

Cette dernière inclusion nous donne en particulier que $\eta=\gamma \gamma^{-1} \eta \in B$. Considérons à présent $\xi \in 3 B$. En usant des propriétés élémentaires du cocycle $\beta$, on vérifie l'identité :

$$
\beta_{\xi}\left(x, \gamma \phi \gamma^{-1} x\right)=\beta_{\gamma^{-1} \xi}(x, \phi x)+\beta_{\gamma^{-1} \xi}\left(\gamma^{-1} x, x\right)-\beta_{\phi^{-1} \gamma^{-1} \xi}\left(\gamma^{-1} x, x\right) .
$$

Comme $\gamma^{-1} \xi \in B_{x}(a, r)$ (par ce qui précède), la condition (ii) nous assure que

$$
\left|\beta_{\gamma^{-1} \xi}(x, \phi x)-L\right|<\varepsilon / 2 \text {. }
$$

Ensuite,

$$
d_{x}\left(\phi^{-1} \gamma^{-1} \xi, a\right)=d_{\phi x}\left(\gamma^{-1} \xi, a\right)=e^{\frac{1}{2}\left[\beta_{\gamma^{-1} \xi}(x, \phi x)+\beta_{a}(x, \phi x)\right]} d_{x}\left(\gamma^{-1} \xi, a\right)<e^{L+\varepsilon / 4} r,
$$

d'où

$$
\left|\beta_{\gamma^{-1} \xi}\left(\gamma^{-1} x, x\right)-\beta_{\phi^{-1} \gamma^{-1} \xi}\left(\gamma^{-1} x, x\right)\right|<\varepsilon / 2
$$

grâce à (i). Finalement, on a obtenu que

$$
\left|\beta_{\xi}\left(x, \gamma \phi \gamma^{-1} x\right)-L\right|<\varepsilon .
$$

De façon en tout point analogue, on vérifie que

$$
\left|\beta_{\xi}\left(x, \gamma \phi^{-1} \gamma^{-1} x\right)+L\right|<\varepsilon .
$$

Ceci finit de prouver que $B \in \mathcal{B}$. 
Nous voici en mesure d'établir, comme annoncé, que la famille de boules $\mathcal{B}$ est dérivante pour la mesure $\mu_{x}$, c'est-à-dire que pour toute fonction borélienne $h: \partial X \rightarrow \mathbb{R}$, on a $\mu_{x}$-presque partout $h^{*}=h$, avec $h^{*}$ désignant la fonction (dite maximale) définie, grâce à ce qui précède, pour tout $\xi \in \Lambda_{M}\left(\Gamma_{0}\right)$ et donc pour $\mu_{x}$-presque tout $\xi \in \partial X$, par

$$
h^{*}(\xi)=\lim _{\rho \rightarrow 0} \sup _{B \in \mathcal{B}_{\rho}, B \ni \xi} \frac{1}{\mu_{x}(B)} \int_{B} h d \mu_{x},
$$

où l'on a noté $\mathcal{B}_{\rho}$ la sous-famille des boules de $\mathcal{B}$ de $d_{x}$-rayons inférieurs à $\rho>0$ (la limite ci-dessus est décroissante). Cette propriété étant avérée dans le cas particulier où $h$ est continue, le cas général en découle par approximation de $h$ dans l'espace $L^{1}\left(\mu_{x}\right)$ par des fonctions continues, grâce à l'inégalité $(*)$ suivante, d'aspect familier, que nous allons incessamment justifier : pour $\alpha>0$,

$$
\mu_{x}\left(\left\{h^{*}>\alpha\right\}\right) \leqslant \frac{e^{\delta(L+\varepsilon)}}{\alpha} \int_{\partial X}|h| d \mu_{x} .
$$

En effet, l'approximation de $h$ par des fonctions continues sous le contrôle de l'inégalité (*) ci-dessus montre que $\mu_{x}\left(\left\{h^{*}-h>\alpha\right\}\right) \leqslant \alpha$ pour $\alpha>0$, d'où $\mu_{x}$-p.p l'inégalité $h^{*} \leqslant h$; de même, $(-h)^{*} \leqslant-h$. Or il est aisé de voir en examinant la définition de la fonction maximale que $-(-h)^{*} \leqslant h^{*}$. En définitive, $h^{*}=h\left(\mu_{x}\right.$-p.p).

Achevons la présente démonstration par la preuve de l'inégalité $(*)$. Prenons une sous-famille $\mathcal{B}^{\prime} \subset \mathcal{B}$ recouvrant $\left\{h^{*}>\alpha\right\}$ telle que pour tout $B \in \mathcal{B}^{\prime}$, on a

$$
\frac{1}{\mu_{x}(B)} \int_{B} h d \mu_{x}>\alpha \text {. }
$$

Choisissons un quelconque compact $K \subset\left\{h^{*}>\alpha\right\}$, puis extrayons de $\mathcal{B}^{\prime}$ une sousfamille finie $\mathcal{B}^{\prime \prime}$ recouvrant $K$. Nommons $B_{1}, B_{2}, \ldots$ les boules de $\mathcal{B}^{\prime \prime}$ rangées par rayon décroissant. Définissons par récurrence (finie)

$$
i_{1}=1, \quad i_{k+1}=\min \left\{i>i_{k} \mid B_{i} \cap\left(B_{i_{1}} \cup \cdots \cup B_{i_{k}}\right)=\varnothing\right\}
$$

tant que possible. Soit $\mathcal{B}^{*}$ la famille des boules $B_{i_{1}}, B_{i_{2}}, \ldots$ Les boules de $\mathcal{B}^{*}$ sont alors deux à deux disjointes et l'on a

$$
\bigcup_{B \in \mathcal{B}^{*}} 3 B \supset \bigcup_{B \in \mathcal{B}^{\prime \prime}} B \supset K .
$$

Considérons $B \in \mathcal{B}^{*}$. Cette boule $B$ est de centre $\gamma a$ pour un $\gamma \in \Gamma_{0}$. Or $3 B \subset$ $\gamma \phi^{-1} \gamma^{-1} B$, puisque pour $\xi \in 3 B$, on a

$$
\begin{aligned}
d_{x}\left(\gamma \phi \gamma^{-1} \xi, \gamma a\right) & =d_{\gamma \phi^{-1} \gamma^{-1} x}(\xi, \gamma a) \\
& =e^{\frac{1}{2}\left[\beta_{\xi}\left(x, \gamma \phi^{-1} \gamma^{-1} x\right)+\beta_{\gamma a}\left(x, \gamma \phi^{-1} \gamma^{-1} x\right)\right]} d_{x}(\xi, \gamma a)<e^{-L+\varepsilon / 2} 3 r<r
\end{aligned}
$$

(rappelons que l'on a $L>\log 3+1$ ). On en déduit que

$$
\mu_{x}(3 B) \leqslant \mu_{x}\left(\gamma \phi^{-1} \gamma^{-1} B\right)
$$


En utilisant le fait que $\gamma \phi \gamma^{-1} \in \Gamma$, on voit que cette dernière quantité vaut

$$
\mu_{\gamma \phi \gamma^{-1} x}(B)=\int_{B} e^{\delta \beta_{\xi}\left(x, \gamma \phi \gamma^{-1} x\right)} d \mu_{x}(\xi)<e^{\delta(L+\varepsilon)} \mu_{x}(B) .
$$

D'où il vient successivement :

$$
\begin{aligned}
\mu_{x}(K) \leqslant \sum_{B \in \mathcal{B}^{*}} \mu_{x}(3 B) & \leqslant e^{\delta(L+\varepsilon)} \sum_{B \in \mathcal{B}^{*}} \mu_{x}(B) \\
& \leqslant \frac{e^{\delta(L+\varepsilon)}}{\alpha} \sum_{B \in \mathcal{B}^{*}} \int_{B} h d \mu_{x} \leqslant \frac{e^{\delta(L+\varepsilon)}}{\alpha} \int_{\partial X}|\varphi| d \mu_{x}
\end{aligned}
$$

(les boules de $\mathcal{B}^{*}$ sont deux à deux disjointes). Comme le choix du compact $K \subset\left\{h^{*}>\alpha\right\}$ était arbitraire, nous en déduisons l'inégalité $(*)$. 


\section{CHAPITRE 3}

\section{MÉLANGE DU FLOT GÉODÉSIQUE}

Désormais, on supposera que le spectre des longueurs de $\Gamma$ n'est pas arithmétique, afin de disposer de l'acquis du chapitre précédent. Au paragraphe 1D sont signalées quelques situations où cette hypothèse est systématiquement satisfaite.

Le principal objet de ce chapitre est d'établir en toute généralité la propriété de mélange du flot géodésique sur $S X / \Gamma$ relativement à une mesure de Bowen-MargulisSullivan associée à une densité conforme de dimension $\delta(\Gamma)$ (théorème 3.1 ). Nous verrons ici que ce résultat découle rapidement du corollaire 2.3. C'est que mélange du flot géodésique et ergodicité du feuilletage horosphérique sont dans une large mesure liés. On trouvera confirmation de ce fait, pour des mesures de BMS finies, dans le sixième chapitre de cet article, où l'on établit à partir du mélange du flot géodésique un théorème ergodique de convergence de moyennes sur les horosphères (théorème 6.1), qui implique en retour l'ergodicité du feuilletage horosphérique. Les théorèmes 3.3 et 3.4 ne font que compléter leur antécédent; le premier énonce le mélange de tout degré, et le second décrit le comportement mélangeant de toutes les autres mesures de BMS, quelles que soient les dimensions des densités conformes associées. Signalons que ces raffinements ne nous seront d'aucune utilité par la suite.

Il convient avant toute chose de préciser ce que nous entendons généralement par mélange d'un flot $\left\{G^{t}\right\}_{t \in \mathbb{R}}$ relativement à une mesure positive $M$. Si $M$ est finie, le flot $\left\{G^{t}\right\}_{t \in \mathbb{R}}$ est dit (fortement) mélangeant si pour tous $A, B$ mesurables, on a

$$
M\left(A \cap G^{-t} B\right) \longrightarrow \frac{M(A) M(B)}{\|M\|} \text { quand } t \longrightarrow \pm \infty .
$$

Si cette définition est familière en mesure finie $(c f .[\mathbf{W}])$, il n'en va plus de même dès que l'on vient à considérer une mesure infinie. Krengel et Sucheston ont proposé dans [Kre-S] une généralisation de cette notion de mélange fort. En mesure infinie, elle coïncide avec la propriété dite de type zéro, antérieurement décrite dans $[\mathbf{H}-\mathbf{K}]$, et s'énonce comme suit : le flot $\left\{G^{t}\right\}_{t \in \mathbb{R}}$ sera dit mélangeant relativement à la mesure 
infinie $M$ si pour tous $A, B$ mesurables et de masses finies, on a $M\left(A \cap G^{-t} B\right) \rightarrow 0$ quand $t \rightarrow \pm \infty$. Les auteurs de $[$ Kre-S $]$ ont avancé divers arguments tendant à bien faire voir que ce concept peut être reçu comme la généralisation naturelle de la notion de mélange (fort), du moins tant que l'on se restreint au seul cadre de la théorie de la mesure. Car il apparaît d'emblée que le mélange en mesure infinie est une propriété bien faible à côté de ce qu'il signifie en mesure finie, et c'est pourquoi un autre type de généralisation a été conçu dans [Ho] et [Kri], mais qui fait appel à une topologie sous-jacente à l'espace mesuré considéré - le théorème 3.4 plus loin viendra à point nommé pour illustrer ce genre de comportement. Faisons enfin observer que s'il est bien connu et même évident que mélange fort implique ergodicité en mesure finie, il n'en est cependant plus rien en mesure infinie, quelle que soit la notion de mélange généralisé considérée. S'il est une façon simple de s'en convaincre, c'est bien en constatant qu'un flot complètement dissipatif est toujours mélangeant (de type zéro). Cela constitue la part triviale du théorème 3.1 ci-dessous, où cette situation correspond aux groupes de type convergent; le cas divergent découlera quant à lui du corollaire 2.3 .

Le mélange fort du flot géodésique en mesure finie a été souvent prouvé dans diverses situations particulières, depuis les surfaces à courbure constante et de volume fini ( $c f .[\mathbf{H e}]$ ) jusqu'aux groupes convexe-cocompacts, et même géométriquement finis en courbure constante $(c f .[\mathbf{R u}])$ - mais l'on sait aujourd'hui que la classe des groupes admettant une mesure de BMS finie excède largement celle des groupes géométriquement finis qui en possèdent une (voir $[\mathbf{A n c}]$ et $[\mathbf{P e i}]$ ). Une preuve générale du mélange fort du flot géodésique en mesure finie est donnée dans [Ro1], qui est différente de celle exposée ci-dessous, mais plus laborieuse. Nous tenons à signaler un récent et élégant argument de M. Babillot (voir [Bab1]) relevant de la théorie spectrale qui permet également d'obtenir le théorème 3.1.

THÉORÈme 3.1. - Le flot géodésique $\left\{g_{\Gamma}^{t}\right\}_{t \in \mathbb{R}}$ est mélangeant relativement à toute mesure de Bowen-Margulis-Sullivan $m_{\Gamma}$ associée à une densité conforme de dimension $\delta(\Gamma)$ et invariante par $\Gamma$. Autrement dit, pour tous $A, B$ boréliens de $S X / \Gamma$, de mesures finies,

$$
m_{\Gamma}\left(A \cap g_{\Gamma}^{-t} B\right) \longrightarrow \frac{m_{\Gamma}(A) m_{\Gamma}(B)}{\left\|m_{\Gamma}\right\|} \quad \text { quand } t \longrightarrow \pm \infty
$$

où la quantité limite est nulle quand $\left\|m_{\Gamma}\right\|$ est infinie.

Comme par la suite nous travaillerons surtout dans le revêtement universel $S X$, il convient de signaler d'ores et déjà que le théorème ci-dessus y prend la forme suivante : pour tous boréliens $A$ et $B$ bornés dans $S X$,

$$
\sum_{\gamma \in \Gamma} m\left(A \cap g^{-t} \gamma B\right) \longrightarrow \frac{m_{\Gamma}(A) m_{\Gamma}(B)}{\left\|m_{\Gamma}\right\|} \text { quand } t \longrightarrow \pm \infty .
$$


Cela est en réalité presque immédiat, pourvu seulement que l'on se souvienne que dans le cas $\left\|m_{\Gamma}\right\|<\infty$, l'ensemble des points fixes de $\Gamma$ est négligeable pour $m$ (voir corollaire 1.8).

Démonstration. - Le cas où est $\Gamma$ est de type convergent est trivial, puisque le flot géodésique est alors complètement dissipatif (théorème 1.7).

Supposons désormais $\Gamma$ de type divergent. Nous allons prouver le théorème sous la forme équivalente suivante : pour toute fonction $\varphi$ dans l'espace $L^{2}\left(m_{\Gamma}\right)$ des fonctions de carré intégrable, $\varphi \circ g_{\Gamma}^{t}$ converge faiblement dans $L^{2}\left(m_{\Gamma}\right)$ vers la constante

$$
\frac{1}{\left\|m_{\Gamma}\right\|} \int \varphi d m_{\Gamma}
$$

quand $t \rightarrow \pm \infty$. Il est suffisant de prouver l'assertion précédente pour $\varphi$ continue à support compact et pour $t$ tendant vers $+\infty$.

La famille $\left\{\varphi \circ g_{\Gamma}^{t}\right\}_{t \in \mathbb{R}}$ étant bornée dans $L^{2}\left(m_{\Gamma}\right)$, on est ramené à montrer qu'elle n'admet de valeurs d'adhérence faible que la constante ci-dessus. Considérons donc une limite faible $f$ d'une suite $\left\{\varphi \circ g_{\Gamma}^{t_{i}}\right\}$ où $t_{i}$ tend vers $+\infty$ avec $i$. D'après le théorème de Banach-Saks (cf. [Ri-N]), on peut extraire une sous-suite $\left\{s_{j}\right\}$ de $\left\{t_{i}\right\}$ telle que

$$
f_{n}=\frac{1}{n} \sum_{j=1}^{n} \varphi \circ g_{\Gamma}^{s_{j}}
$$

converge (fortement) dans $L^{2}\left(m_{\Gamma}\right)$ vers la fonction $f$ quand $n \rightarrow+\infty$. Par un autre théorème classique, on dispose alors d'une sous-suite $\left\{f_{n_{k}}\right\}$ qui converge $m_{\Gamma}$-presque partout vers $f$ quand $k \rightarrow+\infty$. Or on vérifie aisément, en utilisant l'uniforme continuité de $\varphi$, que la suite $f_{n_{k}}$ relevée sur $S X$ converge sur un borélien $E \subset S X$ invariant (stricto sensu) par le feuilletage horosphérique stable et par $\Gamma$, et que la limite $f^{*}$ de cette suite, définie sur $E$, est pareillement invariante. Par ce qui précède, le borélien $E$ est de $m$-mesure pleine, et $f^{*}$ est égale presque partout à $f$ relevée sur $S X$. La fonction $f^{*}$ induit donc une fonction sur $\mathcal{H}$ définie $\widehat{\mu}$-presque partout et invariante par $\Gamma$, et par conséquent essentiellement constante d'après le corollaire 2.3 . Il en résulte que $f^{*}$ est $m$-essentiellement constante, puis que $f$ est $m_{\Gamma}$-essentiellement constante. Si $m_{\Gamma}$ est infinie, nécessairement $f=0$ puisque $f$ est aussi de carré intégrable; si $m_{\Gamma}$ est finie, on a

$$
\int f d m_{\Gamma}=\lim \int f_{k} d m_{\Gamma}=\int \varphi d m_{\Gamma}
$$

(on a utilisé l'invariance de $m_{\Gamma}$ par $g_{\Gamma}^{s_{j}}$ ), d'où l'on tire la valeur attendue de la constante $f$. Il en ressort que $\varphi \circ g_{\Gamma}^{t}$ converge faiblement dans $L^{2}\left(m_{\Gamma}\right)$ vers la constante annoncée, ce qu'il fallait montrer.

Tous les résultats à venir au fil de cet article dépendent du mélange du flot géodésique. Pour établir un certain nombre d'entre eux (théorèmes 3.4, 6.1, 6.4 et 4.2), on 
préférera au théorème 3.1 un énoncé équivalent, où le mélange est conditionné sur les horosphères instables du flot géodésique : c'est le corollaire suivant.

Corollaire 3.2. - Soit $m_{\Gamma}$ une mesure de Bowen-Margulis-Sullivan associée à une densité $\mu$ conforme, invariante par $\Gamma$ et non atomique. Pour tout $u \in S X$, pour tout borélien $E^{+} \subset H^{+}(u)$ et tout borélien $A \subset S X$ borné et de bord m-négligeable, on a

$$
\sum_{\gamma \in \Gamma} \mu_{H^{+}(u)}\left(E^{+} \cap g^{-t} \gamma A\right) \longrightarrow \mu_{H^{+}(u)}\left(E^{+}\right) \frac{m(A)}{\left\|m_{\Gamma}\right\|} \quad \text { quand } t \longrightarrow+\infty
$$

pourvu que la quantité limite ci-dessus ait un sens.

Il faut bien noter que dans l'énoncé, le temps $t$ tend vers $+\infty$ et non vers $-\infty$.

On dispose bien entendu d'un énoncé similaire en considérant le feuilletage stable : si $E^{-} \subset H^{-}(u)$, on a, quand $t \rightarrow+\infty$,

$$
\sum_{\gamma \in \Gamma} \mu_{H^{-}(u)}\left(E^{-} \cap g^{t} \gamma A\right) \longrightarrow \mu_{H^{-}(u)}\left(E^{-}\right) \frac{m(A)}{\left\|m_{\Gamma}\right\|} .
$$

D'autre part, le corollaire 3.2 peut être formulé en termes de fonctions continues à support compact plutôt que de boréliens bornés de bord négligeable (ce que l'on voit aisément à l'aide d'un argument d'approximation) :

Pour tout $u \in S X$, pour tout borélien $E^{+} \subset H^{+}(u)$ et toute fonction $h \in C_{c}(S X / \Gamma)$, que l'on relève en une fonction $\widetilde{h}$ sur $S X$, on a

$$
\int_{E^{+}} \widetilde{h} \circ g^{t} d \mu_{H^{+}(u)} \longrightarrow \mu_{H^{+}(u)}\left(E^{+}\right) \frac{1}{\left\|m_{\Gamma}\right\|} \int \varphi d m_{\Gamma} \quad \text { quand } t \longrightarrow+\infty .
$$

Démonstration. - Nous nous limiterons au cas où $\left\|m_{\Gamma}\right\|<\infty$ (on dispose alors du corollaire 1.8), le complémentaire s'obtenant exactement de la même manière, avec seulement bien des simplifications. Considérons $u \in S X$ et $A$ comme dans l'énoncé. Fixons $\varepsilon>0$. Nous allons prouver que pour $v \in H^{+}(u)$, il existe $r>0$ tel que pour tout borélien $E^{+} \subset B^{+}(v, r)$ on a, dès que $t$ est assez grand,

$$
e^{-\varepsilon} \mu_{H^{+}(u)}\left(E^{+}\right) \frac{m(A)-\varepsilon}{\left\|m_{\Gamma}\right\|} \leqslant \sum_{\gamma \in \Gamma} \mu_{H^{+}(u)}\left(E^{+} \cap g^{-t} \gamma A\right) \leqslant e^{\varepsilon} \mu_{H^{+}(u)}\left(E^{+}\right) \frac{m(A)+\varepsilon}{\left\|m_{\Gamma}\right\|} .
$$

Pour achever la démonstration, il ne restera plus qu'à étendre la validité de cette inégalité à tout $E^{+}$borné dans $H^{+}(u)$ par le biais d'un recouvrement fini de $E^{+}$par des boules du type $B^{+}(v, r)$ comme ci-dessus; on fera ensuite tendre $\varepsilon$ vers 0 , et l'on pourra conclure.

Considérons donc $v \in H^{+}(u)$. L'assertion énoncée précédemment découlera d'une conjonction du théorème 3.1 et du lemme 1.15. Cependant, une légère difficulté survient à l'application de ce lemme, comme le lecteur pourra s'en rendre compte dans ce qui suit, à savoir que $v$ n'est pas nécessairement dans le support de $\mu_{H^{-}(v)}$. C'est 
pour obvier à cet inconvénient que nous introduisons un point $v^{\prime} \in H^{-}(v)$ contenu dans le support de $\mu_{H^{-}(v)}$. Nous rapprocherons pour ainsi dire $v$ et $v^{\prime}$ en les poussant par le flot géodésique.

Grâce aux hypothèses se rapportant à $A$, on peut trouver $\alpha \in] 0, \varepsilon / 3[$ tel que

$$
m\left(A_{\alpha}\right)-\varepsilon \leqslant m(A) \leqslant m\left(A_{-\alpha}\right)+\varepsilon
$$

(la notation $A_{ \pm \alpha}$ est celle introduite au paragraphe $\left.1 \mathrm{H}\right)$. Prenons alors $r_{1}>0$ et $r_{3}>0$ assez petits pour que l'énoncé du lemme 1.15 soit valable pour la mesure $m$ et avec $\alpha$ à la place de $\varepsilon$, puis $s \geqslant 0$ assez grand pour que $g^{s} v^{\prime}$ soit dans la boule $B^{-}\left(g^{s} v, r_{1}\right)$, qui sera notée $E^{-}$; ainsi $\mu_{H^{-}\left(g^{s} v\right)}\left(E^{-}\right)>0$. À présent, si $E^{+}$ est un quelconque borélien contenu dans $B^{+}\left(v, e^{-s}\right)$, alors $g^{s} E^{+} \subset B^{+}\left(g^{s} v, 1\right)$, et une double application du lemme 1.15 nous donne les encadrements suivants, où l'on note $C$ la cellule $C\left(E^{-}, g^{s} E^{+}, r_{3}\right)$ :

$$
e^{-\varepsilon / 3} m(C) \leqslant 2 r_{3} \mu_{H^{-}\left(g^{s} v\right)}\left(E^{-}\right) \mu_{H^{+}\left(g^{s} v\right)}\left(g^{s} E^{+}\right) \leqslant e^{\varepsilon / 3} m(C),
$$

et pour $t \geqslant s$,

$$
\begin{aligned}
e^{-\varepsilon / 3} m\left(C \cap g^{s-t} \gamma A_{-\alpha}\right) & \leqslant 2 r_{3} \mu_{H^{-}\left(g^{s} v\right)}\left(E^{-}\right) \mu_{H^{+}\left(g^{s} v\right)}\left(g^{s} E^{+} \cap g^{s-t} \gamma A\right) \\
& \leqslant e^{\varepsilon / 3} m\left(C \cap g^{s-t} \gamma A_{\alpha}\right) .
\end{aligned}
$$

D'autre part, le théorème 3.1 (pour l'application duquel on prendra note de la remarque qui lui succède) entraîne que pour $t$ assez grand, on a

$$
\sum_{\gamma \in \Gamma} m\left(C \cap g^{s-t} \gamma A_{\alpha}\right) \leqslant e^{\varepsilon / 3}\left\|m_{\Gamma}\right\|^{-1} m(C) m\left(A_{\alpha}\right) \leqslant e^{\varepsilon / 3} m(C)\left\|m_{\Gamma}\right\|^{-1}(m(A)+\varepsilon)
$$

et pareillement

$$
\sum_{\gamma \in \Gamma} m\left(C \cap g^{s-t} \gamma A_{-\alpha}\right) \geqslant e^{-\varepsilon / 3} m(C)\left\|m_{\Gamma}\right\|^{-1}(m(A)-\varepsilon) .
$$

En rassemblant les inégalités précédentes, vu que $\mu_{H^{-}\left(g^{s} v\right)}\left(E^{-}\right)>0$, nous obtenons, pour $t$ assez grand,

$$
\begin{aligned}
e^{-\varepsilon} \mu_{H^{+}\left(g^{s} v\right)}\left(g^{s} E^{+}\right) \frac{m(A)-\varepsilon}{\left\|m_{\Gamma}\right\|} & \leqslant \sum_{\gamma \in \Gamma} \mu_{H^{+}\left(g^{s} v\right)}\left(g^{s} E^{+} \cap g^{s-t} \gamma A\right) \\
& \leqslant e^{\varepsilon} \mu_{H^{+}\left(g^{s} v\right)}\left(g^{s} E^{+}\right) \frac{m(A)+\varepsilon}{\left\|m_{\Gamma}\right\|}
\end{aligned}
$$

Comme $\left(g^{s}\right)^{*} \mu_{H^{+}\left(g^{s} v\right)}$ est proportionnelle à $\mu_{H^{+}(v)}$, l'encadrement désiré s'ensuit.

Nous raffinons à présent sur le théorème 3.1, en montrant avec la même méthode que le flot géodésique est mélangeant de tout degré ( $c f .[\mathbf{W}]$, l'énoncé ci-dessous reproduit la définition de ce concept), ce pour une mesure de BMS finie, la généralisation en mesure infinie se révélant dépourvue d'intérêt. 
THÉORÈme 3.3. - Supposons que $\Gamma$ admette une mesure de Bowen-Margulis-Sullivan finie $m_{\Gamma}$. Pour tout entier $p \geqslant 1$, le flot géodésique $\left\{g_{\Gamma}^{t}\right\}_{t \in \mathbb{R}}$ est mélangeant de degré $p$ relativement à $m_{\Gamma}$. Autrement dit, pour tous boréliens $A_{0}, \ldots, A_{p}$, on a

$$
m_{\Gamma}\left(A_{0} \cap g_{\Gamma}^{-t_{1}} A_{1} \cap \cdots \cap g_{\Gamma}^{-t_{1}-\cdots-t_{p}} A_{p}\right) \longrightarrow \frac{m_{\Gamma}\left(A_{0}\right) \ldots m_{\Gamma}\left(A_{p}\right)}{\left\|m_{\Gamma}\right\|^{p}}
$$

quand $t_{1}, \ldots, t_{p} \rightarrow+\infty$.

Démonstration. - Procédons par récurrence sur $p$. Le cas $p=1$ est donné par le théorème précédent. Supposons acquis le mélange d'ordre $p-1$. De la même manière que dans la preuve du théorème 3.1 , nous allons prouver que

$$
\varphi_{1} \circ g_{\Gamma}^{t_{1}} \ldots \varphi_{p} \circ g_{\Gamma}^{t_{1}+\cdots+t_{p}}
$$

converge faiblement dans $L^{2}\left(m_{\Gamma}\right)$ vers

$$
\left\|m_{\Gamma}\right\|^{-p} \int \varphi_{1} d m_{\Gamma} \ldots \int \varphi_{p} d m_{\Gamma}
$$

quand $t_{1}, \ldots, t_{p} \rightarrow+\infty$, pour $\varphi_{1}, \ldots, \varphi_{p}$ continues à support compact, ce qui suffira. Considérons donc une valeur d'adhérence faible $f$ de cette famille, associée à une suite dans $\mathbb{R}^{p}$ dont chaque composante tend vers $+\infty$. On peut (par le théorème de Banach-Saks) extraire de cette dernière une sous-suite $\left\{\left(s_{1 j}, \ldots, s_{p j}\right)\right\}$ telle que

$$
f_{n}=\frac{1}{n} \sum_{j=1}^{n} \varphi_{1} \circ g_{\Gamma}^{s_{1 j}} \ldots \varphi_{p} \circ g_{\Gamma}^{s_{1 j}+\cdots+s_{p j}}
$$

converge dans $L^{2}\left(m_{\Gamma}\right)$ vers $f$ quand $n \rightarrow+\infty$. En considérant une sous-suite convergeant p.p vers $f$, on montre, comme dans la preuve du théorème 3.1, que $f$ est le quotient d'une fonction sur $S X$ invariante par le feuilletage stable, puis que $f$ est essentiellement constante, grâce au corollaire 2.3. Enfin, on a

$$
\int f d m_{\Gamma}=\lim \int f_{n} d m_{\Gamma}=\lim \frac{1}{n} \sum_{j=1}^{n} \int \varphi_{1} \varphi_{2} \circ g_{\Gamma}^{s_{2 j}} \ldots \varphi_{p} \circ g_{\Gamma}^{s_{2 j}+\cdots+s_{p j}} d m_{\Gamma}
$$

(on a utilisé l'invariance de $m_{\Gamma}$ par $g_{\Gamma}^{s_{1 j}}$ ); or le mélange d'ordre $p-1$ nous fournit la valeur de cette limite, qui est conforme à ce que l'on attendait.

Le théorème suivant décrit le comportement des mesures de BMS laissées à l'écart par le théorème 3.1. On y voit apparaître une façon de mélange. Rappelons que si une mesure du type $m_{\Gamma}^{\nu^{-}, \nu^{+}}$de l'énoncé ci-dessous est finie, alors $\delta^{-}=\delta^{+}=\delta(\Gamma)$ et $m_{\Gamma}^{\nu^{-}, \nu^{+}}=m_{\Gamma}$ est unique (d'après un avatar du théorème 1.7); nous avons donc ici affaire à des mesures en général infinies. Avec la propriété de mélange que manifeste une mesure $m_{\Gamma}^{\nu^{-}, \nu^{+}}$infinie et que l'on établit ci-dessous, l'on retrouve la notion de mélange généralisé suggérée par Hopf (voir [Ho]), illustrée d'abord par lui-même d'un exemple spécialement bâti à cet effet, puis par Krickeberg ([Kri $]$ ) pour certains systèmes dynamiques liés à des chaînes de Markov. Ce type de mélange généralisé est 
plus fort que celui défini dans $[\mathbf{K r e - S}]$ et dont il a été question plus haut à propos du théorème 3.1. Insistons encore sur son caractère topologique : on se convainc sans peine que les conditions de continuité apparaissant dans l'énoncé ci-dessous sont absolument nécessaires dès que $\left(\nu^{-}, \nu^{+}\right) \neq(\mu, \mu)$.

THÉORÈme 3.4. - Soit $m_{\Gamma}=m_{\Gamma}^{\mu, \mu}$ une mesure de Bowen-Margulis-Sullivan associée à une densité $\mu$ conforme de dimension $\delta=\delta(\Gamma)$ et invariante par $\Gamma$. Soient $\nu^{-}$ et $\nu^{+}$deux densités, conformes de dimensions respectives $\delta^{-}$et $\delta^{+}$, invariantes par $\Gamma$, non atomiques et rendant négligeable l'ensemble des points fixes elliptiques de $\Gamma$. Alors, pour tous boréliens bornés $A, B$ de $S X / \Gamma$ tels que $m_{\Gamma}^{\nu^{-}, \mu}(\partial A)=m_{\Gamma}^{\mu, \nu^{+}}(\partial B)=0$, on a

$$
e^{\left(\delta^{+}-\delta\right) t} m_{\Gamma}^{\nu^{-}, \nu^{+}}\left(A \cap g_{\Gamma}^{-t} B\right) \longrightarrow \frac{m_{\Gamma}^{\nu^{-}, \mu}(A) m_{\Gamma}^{\mu, \nu^{+}}(B)}{\left\|m_{\Gamma}\right\|} \quad \text { quand } t \longrightarrow+\infty,
$$

où la quantité limite ci-dessus est nulle quand $\left\|m_{\Gamma}\right\|$ est infinie.

Ce théorème ergodique présente une forme équivalente en termes de fonctions :

Pour toutes fonctions $\varphi, \psi S X / \Gamma \rightarrow \mathbb{R}$, continues à support compact, on a

$$
e^{\left(\delta^{+}-\delta\right) t} \int \varphi \psi \circ g^{t} d m_{\Gamma}^{\nu^{-}, \nu^{+}} \longrightarrow \frac{1}{\left\|m_{\Gamma}\right\|} \int \varphi d m_{\Gamma}^{\nu^{-}, \mu} \int \psi d m_{\Gamma}^{\mu, \nu^{+}} \text {quand } t \longrightarrow+\infty \text {. }
$$

Démonstration. - Nous nous limiterons au cas où $\left\|m_{\Gamma}\right\|<\infty$, le cas complémentaire s'obtenant exactement de la même manière, avec seulement bien des simplifications. Désignons par $\nu$ une densité conforme de dimension $\delta_{\nu}$, invariante par $\Gamma$ et non atomique. Nous allons prouver le théorème successivement dans les cas où $\left(\nu^{-}, \nu^{+}\right)$est d'abord $(\nu, \mu)$, puis $(\mu, \nu)$, et enfin dans le cas général.

Soit $B$ un borélien de $S X$, borné et de bord $m$-négligeable. Dans ces conditions, étant donné $\varepsilon>0$ arbitrairement petit, par application du lemme 1.15 puis du corollaire 3.2 , on obtient que pour toute cellule $C$ assez petite, dès que $t$ est assez grand,

$$
e^{-\varepsilon} \frac{m^{\nu, \mu}(C) m(B)}{\left\|m_{\Gamma}\right\|} \leqslant \sum_{\gamma \in \Gamma} m^{\nu, \mu}\left(C \cap g^{-t} \gamma B\right) \leqslant e^{\varepsilon} \frac{m^{\nu, \mu}(C) m(B)}{\left\|m_{\Gamma}\right\|} .
$$

À l'aide d'un argument d'approximation par les cellules, il vient, pour tout borélien $A$,

$$
\sum_{\gamma \in \Gamma} m^{\nu, \mu}\left(A \cap g^{-t} \gamma B\right) \longrightarrow \frac{m^{\nu, \mu}(A) m(B)}{\left\|m_{\Gamma}\right\|} \text { quand } t \longrightarrow+\infty .
$$

Ensuite, en usant des propriétés de quasi-invariance des mesures de BMS généralisées, on voit que

$$
\begin{aligned}
\sum_{\gamma \in \Gamma} e^{\left(\delta_{\nu}-\delta\right) t} m^{\mu, \nu}\left(A \cap g^{-t} \gamma B\right) & =\sum_{\gamma \in \Gamma} m^{\mu, \nu}\left(B \cap g^{t} \gamma A\right) \\
& =\sum_{\gamma \in \Gamma} m^{\nu, \mu}\left(\mathcal{I} B \cap g^{-t} \gamma \mathcal{I} A\right)
\end{aligned}
$$


Avec ce qui précède, pourvu que $A$ soit de bord $m$-négligeable, on obtient que

$$
e^{\left(\delta_{\nu}-\delta\right) t} \sum_{\gamma \in \Gamma} m^{\mu, \nu}\left(A \cap g^{-t} \gamma B\right) \longrightarrow \frac{m^{\nu, \mu}(\mathcal{I} B) m(\mathcal{I} A)}{\left\|m_{\Gamma}\right\|}=\frac{m(A) m^{\mu, \nu}(B)}{\left\|m_{\Gamma}\right\|}
$$

lorsque $t \rightarrow+\infty$. En examinant à travers le prisme du lemme 1.15 ce que l'on vient d'établir, de la même façon que nous avions déduit le corollaire 3.2 du théorème 3.1 via ce même lemme, nous allons voir que pour tout $u \in S X$, pour tout borélien borné $E^{+} \subset H^{+}(u)$ de bord $\mu_{H^{+}(u)}$-négligeable, et tout borélien borné $B \subset S X$ de bord $m^{\mu, \nu}$-négligeable,

$(*) \quad e^{\left(\delta_{\nu}-\delta\right) t} \sum_{\gamma \in \Gamma} \nu_{H^{+}(u)}\left(E^{+} \cap g^{-t} \gamma B\right) \longrightarrow \mu_{H^{+}(u)}\left(E^{+}\right) \frac{m^{\mu, \nu}(B)}{\left\|m_{\Gamma}\right\|} \quad$ quand $t \longrightarrow+\infty$.

Aussi reprenons pas à pas la preuve du corollaire 3.2, à laquelle nous renvoyons le lecteur. Étant donné $u \in S X, \varepsilon>0$ arbitrairement petit, $v \in H^{+}(u)$, on fixe $v^{\prime} \in \operatorname{supp} \mu_{H^{-}(v)}$; après avoir choisi $\left.\alpha \in\right] 0, \varepsilon / 3[$ assez petit pour que

$$
m^{\mu, \nu}\left(B_{\alpha}\right)-\varepsilon \leqslant m^{\mu, \nu}(B) \leqslant m^{\mu, \nu}\left(B_{-\alpha}\right)+\varepsilon,
$$

on prend $r_{1}, r_{3}>0$ tels que l'énoncé du lemme 1.15 soit valable pour les mesures $m$ et $m^{\mu, \nu}$ avec $\alpha$ à la place de $\varepsilon$, et enfin $s \geqslant 0$ tel que $g^{s} v^{\prime} \in B^{-}\left(g^{s} v, r_{1}\right)=E^{-}$. Quitte à modifier $r_{1}$, on peut supposer de plus que $\mu_{H^{-}\left(g^{s} v\right)}\left(\partial E^{-}\right)=0$. Pour un quelconque borélien $E^{+} \subset B^{+}\left(v, e^{-s}\right)$ borné et de bord $\mu_{H^{+}(u)}$-négligeable, on a, par application du lemme 1.15, en notant $C=C\left(E^{-}, g^{s} E^{+}, r_{3}\right)$,

$$
e^{-\varepsilon / 3} m(C) \leqslant 2 r_{3} \mu_{H^{-}\left(g^{s} v\right)}\left(E^{-}\right) \mu_{H^{+}\left(g^{s} v\right)}\left(g^{s} E^{+}\right) \leqslant e^{\varepsilon / 3} m(C),
$$

et, pour $t \geqslant s$,

$$
\begin{aligned}
e^{-\varepsilon / 3} m^{\mu, \nu}\left(C \cap g^{s-t} \gamma B_{-\alpha}\right) & \leqslant 2 r_{3} \mu_{H^{-}\left(g^{s} v\right)}\left(E^{-}\right) \nu_{H^{+}\left(g^{s} v\right)}\left(g^{s} E^{+} \cap g^{s-t} \gamma B\right) \\
& \leqslant e^{\varepsilon / 3} m^{\mu, \nu}\left(C \cap g^{s-t} \gamma B_{\alpha}\right) .
\end{aligned}
$$

Grâce à ce qui a été établi plus haut, c'est-à-dire le cas $\left(\nu^{-}, \nu^{+}\right)=(\mu, \nu)$ du théorème, et compte tenu du fait que $m(\partial C)=0$ par construction de $C$, on sait que, pour $t$ assez grand, on a

$$
\sum_{\gamma \in \Gamma} e^{\left(\delta_{\nu}-\delta\right)(t-s)} m^{\mu, \nu}\left(C \cap g^{s-t} \gamma B_{\alpha}\right) \leqslant e^{\varepsilon / 3} m(C)\left\|m_{\Gamma}\right\|^{-1}\left(m^{\mu, \nu}(B)+\varepsilon\right),
$$

ainsi que

$$
\sum_{\gamma \in \Gamma} e^{\left(\delta_{\nu}-\delta\right)(t-s)} m^{\mu, \nu}\left(C \cap g^{s-t} \gamma B_{-\alpha}\right) \geqslant e^{-\varepsilon / 3} m(C)\left\|m_{\Gamma}\right\|^{-1}\left(m^{\mu, \nu}(B)-\varepsilon\right) .
$$


Il résulte des encadrements précédents que pour $t$ grand, on a

$$
\begin{aligned}
e^{-\varepsilon} e^{-\delta s} \mu_{H^{+}\left(g^{s} v\right)}\left(g^{s} E^{+}\right) \frac{m^{\mu, \nu}(B)-\varepsilon}{\left\|m_{\Gamma}\right\|} & \leqslant e^{\left(\delta_{\nu}-\delta\right) t} e^{-\delta_{\nu} s} \sum_{\gamma \in \Gamma} \nu_{H^{+}\left(g^{s} v\right)}\left(g^{s} E^{+} \cap g^{s-t} \gamma B\right) \\
& \leqslant e^{\varepsilon} e^{-\delta s} \mu_{H^{+}\left(g^{s} v\right)}\left(g^{s} E^{+}\right) \frac{m^{\mu, \nu}(B)+\varepsilon}{\left\|m_{\Gamma}\right\|}
\end{aligned}
$$

puis

$$
\begin{aligned}
e^{-\varepsilon} \mu_{H^{+}(u)}\left(E^{+}\right) \frac{m^{\mu, \nu}(B)-\varepsilon}{\left\|m_{\Gamma}\right\|} & \leqslant e^{\left(\delta_{\nu}-\delta\right) t} \sum_{\gamma \in \Gamma} \nu_{H^{+}(u)}\left(E^{+} \cap g^{-t} \gamma B\right) \\
& \leqslant e^{\varepsilon} \mu_{H^{+}(v)}\left(E^{+}\right) \frac{m^{\mu, \nu}(B)+\varepsilon}{\left\|m_{\Gamma}\right\|} .
\end{aligned}
$$

Ce dernier encadrement subsiste maintenant pour tout $E^{+} \subset H^{+}(u)$ borné et de bord négligeable, et il ne reste plus qu'à faire tendre $\varepsilon$ vers 0 pour achever d'établir $(*)$.

Le théorème découle finalement de la conjonction de $(*)$ où l'on mettra $\nu^{+}$à la place de $\nu$, et du lemme 1.15 appliqué à la mesure $m^{\nu^{-}, \nu^{+}}$, de la même façon que l'on a tiré le cas $\left(\nu^{-}, \nu^{+}\right)=(\nu, \mu)$ du corollaire 3.2 et dudit lemme, en début de preuve. 



\section{CHAPITRE 4}

\section{DÉNOMBREMENT ET ÉQUIDISTRIBUTION ASYMPTOTIQUE DES ORBITES}

Le présent chapitre est dévolue au problème du dénombrement et de la répartition des orbites de $\Gamma$, que nous relierons à celui du mélange du flot géodésique, objet du chapitre précédent, et qui pour nous fut justement suscité par ce qui vient. Rappelons que depuis le chapitre 3, il est supposé que le spectre des longueurs du groupe $\Gamma$ n'est pas arithmétique.

Le résultat principal est le théorème 4.1 (comprenant 4.1.1 et 4.1.2). Les deux corollaires qui font suite à l'énoncé 4.1.1, quoique immédiats, méritent d'être mentionnés explicitement, car ils paraîtront plus accessibles à première vue. Le corollaire 2 , d'abord, montre un équivalent à la fonction orbitale de $\Gamma$ admettant une mesure de BMS finie. Ce problème ayant été abordé bien des fois, nombre de résultats partiels étaient déjà connus, mais nous n'en citerons que très peu. Le lecteur intéressé pourra trouver dans [Bab2] un aperçu historique qui fait défaut ici. Nous nous contenterons d'observer que, hormis dans [Marg] (cas des variétés compactes), il s'en fallait assez que les méthodes jusque-là employées fussent vraiment élémentaires, soit qu'elles relevassent de la théorie spectrale, pour les groupes géométriquement finis dans l'espace hyperbolique de dimension $d$, où l'on rencontre généralement l'obstruction $\delta(\Gamma)>d / 2$ ( $[\mathbf{C d V}],[\mathbf{L}-\mathbf{P}]$ qui établit un développement asymptotique extrêmement poussé), notablement contournée dans [Patt4] (cas des surfaces convexe-cocompactes), soit qu'elles ressortissent à la dynamique symbolique et au formalisme thermodynamique, avec codage du flot géodésique, en courbure pincée ([L] pour les groupes convexecocompacts, [Dal-P] pour quelques autres). En outre, les groupes à mesure de BMS finie jusqu'alors étudiés appartenaient systématiquement à la classe des groupes géométriquement finis, sans d'ailleurs que le problème en question ait pu être résolu pour l'ensemble de ceux-là (sauf en dimension 2 et courbure constante), et l'on avait même été jusqu'à conjecturer (voir [Patt3]) que cette classe était caractérisée par le fait de posséder un équivalent exactement exponentiel pour la fonction orbitale, comme dans le corollaire 2 ci-dessous. Or deux travaux récents dont nous avons déjà fait mention, 
[Anc] et [Pei], ont révélé que la classe des groupes à mesure finie excède très largement la précédente, même en dimension deux et courbure constante. Aussi convient-il de redresser la conjecture évoquée, à la lumière du corollaire 2 , auquel on joindra le théorème 4.1.2 qui en constitue la contrepartie : le fait que la fonction orbitale ait un équivalent exactement exponentiel caractérise la classe des groupes à mesure de BMS finie. À propos du théorème 4.1.2, il semble que le cas où $X$ est le disque hyperbolique et où $\delta(\Gamma)=1$ ait seul été établi auparavant, dans [Patt2]; il faut d'autre part noter que pour certains groupes satisfaisant à l'hypothèse de ce théorème, c'est un équivalent à la fonction orbitale qui a pu être proposé (cas des revêtements abéliens de variétés convexe-cocompactes, voir [Pol-S], [Bab-L]).

Plus que le dénombrement, c'est la répartition asymptotique même des points des orbites d'un groupe à mesure de BMS finie que nous pouvons ici cerner : le corollaire 1 en offre un aperçu, qui exprime essentiellement que la probabilité uniforme sur les points orbitaux situés dans une boule converge vaguement vers la mesure de Patterson-Sullivan, quand le rayon de ladite boule tend vers l'infini (on trouve déjà un tel énoncé dans $[\mathbf{L}]$ et $[\mathbf{P o l}-\mathbf{S}])$. Mais c'est l'équidistribution plus générale qu'avance le théorème 4.1.1 à laquelle nous avons été conduits, puisque cet énoncé est l'exact équivalent du mélange du flot géodésique - nous n'exposerons cependant que l'ascendance du théorème 3.1 , déjà établi, sur le suivant, mais l'on pourrait obtenir la réciproque par les mêmes méthodes que celles de la démonstration. La clef de cette dernière est une correspondance entre mélange du flot géodésique et distribution orbitale (voir la première étape ci-dessous). Nous avions déjà rencontré ce type d'équidistribution double dans [Ro2] (en correspondance avec l'ergodicité rationnelle du flot géodésique). Enfin, nous utiliserons pleinement l'énoncé général 4.1 dans le problème de la répartition des géodésiques fermées qui fera l'objet du chapitre suivant.

On a ci-dessous adopté les notations $\mathcal{D}_{z}$ pour la masse de Dirac au point $z$, et $C(K)^{*}$ pour le dual faible-* de l'espace des fonctions continues sur un espace compact $K$.

ThÉORÈme 4.1.1. - Supposons que $\Gamma$ admette une mesure de Bowen-MargulisSullivan $m_{\Gamma}$ finie, associée à une densité $\mu$ conforme de dimension $\delta=\delta(\Gamma)$ et invariante par $\Gamma$. Alors, pour tous $x$ et $y$ dans $X$, quand $t$ tend vers $+\infty$,

$$
\delta\left\|m_{\Gamma}\right\| e^{-\delta t} \sum_{\substack{\gamma \in \Gamma \\(x, \gamma y) \leqslant t}} \mathcal{D}_{\gamma y} \otimes \mathcal{D}_{\gamma^{-1} x} \rightarrow \mu_{x} \otimes \mu_{y} \quad \text { faiblement dans } C(\bar{X} \times \bar{X})^{*} .
$$

Corollaire 1. $-\delta\left\|m_{\Gamma}\right\| e^{-\delta t} \sum_{\substack{\gamma \in \Gamma \\(x, \gamma o) \leqslant t}} \mathcal{D}_{\gamma_{o}} \rightarrow \mu_{x} \quad$ faiblement dans $C(\bar{X})^{*}$.

Corollaire 2. - $\#\{\gamma \in \Gamma \mid(x, \gamma y) \leqslant t\} \sim \frac{\left\|\mu_{x}\right\|\left\|\mu_{y}\right\|}{\delta\left\|m_{\Gamma}\right\|} e^{\delta t}$. 
THÉORÈmE 4.1.2. - Supposons que $\Gamma$ n'admette pas de mesure de Bowen-MargulisSullivan finie. Alors, quand $t$ tend vers $+\infty$,

$$
\#\{\gamma \in \Gamma \mid(o, \gamma o) \leqslant t\}=o\left(e^{\delta t}\right) .
$$

Démonstration. - Nous nous contenterons de détailler seulement la preuve du théorème 4.1.1; l'énoncé complémentaire 4.1.2 s'obtient de la même manière, en suivant le cours de la présente preuve (on utilisera notamment le théorème 3.1 dans la situation correspondante), mais avec nombre de simplifications, ce qui n'étonnera pas, vu la relative grossièreté du résultat. Supposons donc $\left\|m_{\Gamma}\right\|<\infty$, et rappelons que dans ce cas le support de $\mu$ est $\Lambda(\Gamma)$.

Notons $\nu_{x, y}^{t}$ la mesure de l'énoncé, c'est-à-dire

$$
\nu_{x, y}^{t}=\delta\left\|m_{\Gamma}\right\| e^{-\delta t} \sum_{(x, \gamma y) \leqslant t} \mathcal{D}_{\gamma y} \otimes \mathcal{D}_{\gamma^{-1} x} .
$$

Pour $x \in X$ et $A \subset \partial X$, introduisons deux espèces de cônes de sommet $x$ et de base $A$, l'un par excès, l'autre par défaut, comme suit (avec $r>0$ ) :

$$
\begin{aligned}
& \left.\left.\mathcal{C}_{r}^{+}(x, A)=\left\{y \in X \mid \exists x^{\prime} \in B(x, r) \text { et } \xi \in A \text { tels que } B(y, r) \cap\right] x^{\prime} \xi\right) \neq \varnothing\right\}, \\
& \left.\left.\mathcal{C}_{r}^{-}(x, A)=\left\{y \in X \mid B(y, r) \subset \bigcap_{x^{\prime} \in B(x, r)} \bigcup_{\xi \in A}\right] x^{\prime} \xi\right)\right\} .
\end{aligned}
$$

Dans une première étape, où sera mise en lumière la correspondance entre mélange du flot géodésique (fait acquis par le théorème 3.1) et dénombrement asymptotique des orbites de $\Gamma$, nous approcherons le comportement asymptotique quand $T \rightarrow+\infty$ de $\nu_{x, y}^{T}\left(\mathcal{C}_{r}^{ \pm}(x, A) \times \mathcal{C}_{r}^{ \pm}(y, B)\right)$, pour $A, B$ assez petits, et $x, y$ convenablement situés par rapport à $A$ et $B$. Une seconde étape affranchira le résultat précédent de la condition portant sur $x$ et $y$, puis une dernière achèvera la preuve du théorème par globalisation par rapport à $A$ et $B$.

Première étape. - Soit $\varepsilon>0$. Soient $\left(\xi_{0}, \eta_{0}\right) \in \partial X \times \partial X$ et $x, y \in X$. Supposons que $x \in\left(\xi_{0} \xi_{0}^{*}\right)$ et $y \in\left(\eta_{0} \eta_{0}^{*}\right)$ où $\xi_{0}^{*}$ et $\eta_{0}^{*}$ sont dans $\Lambda(\Gamma)$. Il existe alors des voisinages ouverts $V$ et $W$ respectivement de $\xi_{0}$ et $\eta_{0}$ dans $\partial X$, tels que pour tous boréliens $A \subset V$ et $B \subset W$, on a, quand $T \rightarrow+\infty$,

$$
\begin{aligned}
& \limsup \nu_{x, y}^{T}\left(\mathcal{C}_{1}^{-}(x, A) \times \mathcal{C}_{1}^{-}(y, B)\right) \leqslant e^{\varepsilon} \mu_{x}(A) \mu_{y}(B) \\
& \text { et } \liminf \nu_{x, y}^{T}\left(\mathcal{C}_{1}^{+}(x, A) \times \mathcal{C}_{1}^{+}(y, B)\right) \geqslant e^{-\varepsilon} \mu_{x}(A) \mu_{y}(B) .
\end{aligned}
$$

Démonstration. - Commençons par introduire un certain nombre d'objets géométriques dont nous aurons besoin tout au long de la preuve de cette étape. On vérifiera aisément que tous ces ensembles ont une structure stable par les isométries de $X$.

D'abord, nous allons définir, pour $z \in X, r>0, A \subset \partial X$, l'ensemble $K^{+}(z, r, A)$ des points de $S X$ qui déterminent chacun une géodésique d'extrémité positive dans $A$ et qui sont situés sur un intervalle de cette géodésique de longueur $r$ et de milieu basé dans $X$ à distance au plus $r$ de $z$. En voici la description précise : pour $(\xi, \eta) \in \partial^{2} X$, 
notons $z_{\xi \eta}$ le point de $S X$ déterminé par les conditions $g^{-\infty} z_{\xi \eta}=\xi, g^{+\infty} z_{\xi \eta}=\eta$ et le projeté de $z_{\xi \eta}$ dans $X$ est le point de la géodésique $(\xi \eta)$ le plus proche de $z$ (il s'agit du «projeté orthogonal » de $z \operatorname{sur}(\xi \eta), c f .1 \mathrm{~A})$; on définit alors $K^{+}(z, r, A)$ comme l'ensemble des points $g^{s} z_{\xi \eta}$ où $-r / 2<s<r / 2$ et où $(\xi, \eta) \in \partial^{2} X$ est tel que $\eta \in A$ et que la distance de $z$ à la géodésique $(\xi \eta)$ est strictement inférieure à $r$. On utilisera aussi l'ensemble symétrique du précédent obtenu par inversion des extrémités des géodésiques, à savoir $K^{-}(z, r, A)=\mathcal{I} K^{+}(z, r, A)$. Nous noterons aussi $K(z, r)=K^{ \pm}(z, r, \partial X)$. Remarquons que $K(z, r) \subset S B(z, 3 r / 2)$.

D'autre part, nous ne pourrons nous contenter de l'ombre à l'infini d'une boule de $X$ telle qu'elle a été définie en $1 \mathrm{~A}$, mais aurons besoin des variantes ci-dessous de cet objet. Pour $r>0, a \in X$ et $b \in X$, avec $(a, b)>2 r$, nous distinguerons les ombres suivantes, respectivement par excès et par défaut :

$$
\begin{aligned}
& \left.\left.\mathcal{O}_{r}^{+}(a, b)=\left\{\xi \in \partial X \mid \exists a^{\prime} \in B(a, r) \text { tel que }\right] a^{\prime} \xi\right) \cap B(b, r) \neq \varnothing\right\}, \\
& \left.\left.\mathcal{O}_{r}^{-}(a, b)=\left\{\xi \in \partial X \mid \forall a^{\prime} \in B(a, r),\right] a^{\prime} \xi\right) \cap B(b, r) \neq \varnothing\right\} .
\end{aligned}
$$

Quand $a$ tend vers un point $\eta \in \partial X$, les ombres précédentes ont pour limite commune :

$$
\mathcal{O}_{r}(\eta, b)=\{\xi \in \partial X \mid(\eta \xi) \cap B(b, r) \neq \varnothing\}=\mathcal{O}_{r}^{ \pm}(\eta, b) .
$$

Enfin, pour $r>0$ et $a, b \in X$ avec $(a, b)>2 r$, nous noterons $\mathcal{L}_{r}(a, b)$ l'ensemble des $(\xi, \eta) \in \partial^{2} X$ tels que la géodésique $(\xi \eta)$ orientée de $\xi$ vers $\eta$ traverse d'abord $B(a, r)$ et ensuite $B(b, r)$. Observons que

$$
\mathcal{O}_{r}^{-}(b, a) \times \mathcal{O}_{r}^{-}(a, b) \subset \mathcal{L}_{r}(a, b) \subset \mathcal{O}_{r}^{+}(b, a) \times \mathcal{O}_{r}^{+}(a, b) .
$$

Choisissons à présent $r \in] 0, \min (1, \varepsilon / 30 \delta)[$ tel que

$$
\mu_{x}\left(\partial \mathcal{O}_{r}\left(\xi_{0}, x\right)\right)=0=\mu_{y}\left(\partial \mathcal{O}_{r}\left(\eta_{0}, y\right)\right)
$$

(ce qui ne fait qu'exclure un ensemble de valeurs de $r$ au plus dénombrable). Comme $\xi_{0}^{*} \in \mathcal{O}_{r}\left(\xi_{0}, x\right)$ et $\eta_{0}^{*} \in \mathcal{O}_{r}\left(\eta_{0}, y\right)$, nous avons

$$
\mu_{x}\left(\mathcal{O}_{r}\left(\xi_{0}, x\right)\right) \mu_{y}\left(\mathcal{O}_{r}\left(\eta_{0}, y\right)\right)>0
$$

Prenons alors deux ouverts $\widehat{V}$ et $\widehat{W}$ de $\bar{X}$, contenant respectivement $\xi_{0}$ et $\eta_{0}$, assez petits afin que pour tout $(a, b) \in \widehat{V} \times \widehat{W}$, l'on ait

$$
\begin{aligned}
& e^{-\varepsilon / 30} \mu_{x}\left(\mathcal{O}_{r}\left(\xi_{0}, x\right)\right) \leqslant \mu_{x}\left(\mathcal{O}_{r}^{ \pm}(a, x)\right) \leqslant e^{\varepsilon / 30} \mu_{x}\left(\mathcal{O}_{r}\left(\xi_{0}, x\right)\right) \\
\text { et } \quad & e^{-\varepsilon / 30} \mu_{y}\left(\mathcal{O}_{r}\left(\eta_{0}, y\right)\right) \leqslant \mu_{y}\left(\mathcal{O}_{r}^{ \pm}(b, y)\right) \leqslant e^{\varepsilon / 30} \mu_{y}\left(\mathcal{O}_{r}\left(\eta_{0}, y\right)\right) .
\end{aligned}
$$

Nous choisissons enfin pour $V$ et $W$ des voisinages ouverts dans $\partial X$ de $\xi_{0}$ et $\eta_{0}$ respectivement, tels que $\bar{V} \subset \widehat{V} \cap \partial X$ et $\bar{W} \subset \widehat{W} \cap \partial X$.

Considérons deux boréliens $A \subset V$ et $B \subset W$. Notons pour alléger

$$
K^{+}=K^{+}(x, r, A) \quad \text { et } \quad K^{-}=K^{-}(y, r, B) .
$$


Notre méthode consiste à estimer asymptotiquement (quand $T \rightarrow+\infty$ ) la quantité

$$
\int_{0}^{T} e^{\delta t} \sum_{\gamma \in \Gamma} m\left(K^{+} \cap g^{-t} \gamma K^{-}\right) d t
$$

en la développant suivant le groupe $\Gamma$, dont les éléments intervenant ici seront justement ceux que l'on cherche à dénombrer dans cette étape. Comme le théorème 3.1 fournit de son côté un équivalent asymptotique de l'intégrande ci-dessus, le résultat de la première étape s'ensuivra.

En revenant aux définitions, on vérifie, pour $\gamma \in \Gamma$ avec $(x, \gamma y)>2 r$, que

$$
m\left(K^{+} \cap g^{-t} \gamma K^{-}\right)=\int \frac{d \mu_{x}(\xi) d \mu_{x}(\eta)}{\left[d_{x}(\xi, \eta)\right]^{2 \delta}} \int_{-r / 2}^{r / 2} \mathbb{1}_{K(\gamma y, r)}\left(g^{t+s} x_{\xi \eta}\right) d s
$$

où l'intégrale porte sur $\mathcal{L}_{r}(x, \gamma y) \cap(\gamma B \times A)$.

Nous allons d'abord majorer la quantité

$$
\int_{0}^{T-3 r} e^{\delta t} \sum_{\gamma \in \Gamma} m\left(K^{+} \cap g^{-t} \gamma K^{-}\right) d t .
$$

D'une part, pour $(\xi, \eta) \in \mathcal{L}_{r}(x, \gamma y)$, on a

$$
d_{x}(\xi, \eta)^{2 \delta} \geqslant e^{-2 \delta r} \geqslant e^{-\varepsilon / 15} .
$$

D'autre part, supposons que $\mathcal{L}_{r}(x, \gamma y) \cap(\gamma B \times A)$ ne soit pas vide; en se reportant aux définitions, on voit aisément que cela entraîne que $\gamma y \in \mathcal{C}_{1}^{+}(x, A)$ (nous avions pris le soin d'avoir $r<1$ ) ; de plus, en faisant agir $\gamma^{-1}$, on a, de façon symétrique, que $\mathcal{L}_{r}\left(\gamma^{-1} x, y\right) \cap\left(B \times \gamma^{-1} A\right)$ n'est pas vide, d'où $\gamma^{-1} x \in \mathcal{C}_{1}^{+}(y, B)$. Rappelons aussi que $\mathcal{L}_{r}(x, \gamma y) \subset \mathcal{O}_{r}^{+}(\gamma y, x) \times \mathcal{O}_{r}^{+}(x, \gamma y)$.

Enfin, pour $(\xi, \eta) \in \mathcal{L}_{r}(x, \gamma y),|s|<r / 2$, et $T>0$, on voit, en examinant la définition de $K(\gamma y, r)$, que

$$
\int_{0}^{T-3 r} e^{\delta t} \mathbb{1}_{K(\gamma y, r)}\left(g^{t+s} x_{\xi \eta}\right) d t \begin{cases}\leqslant & e^{\delta 3 r} r e^{\delta(x, \gamma y)} \leqslant e^{\varepsilon / 10} r e^{\delta(x, \gamma y)} \\ =0 & \text { si }(x, \gamma y)>T .\end{cases}
$$

Il ressort de tous ces faits réunis la majoration $(* 1)$ suivante, avec $c_{1}$ désignant une constante indépendante de $T>0$ :

$$
\begin{aligned}
\int_{0}^{T-3 r} e^{\delta t} \sum_{\gamma \in \Gamma} m\left(K^{+}\right. & \left.\cap g^{-t} \gamma K^{-}\right) d t \\
& \leqslant e^{\varepsilon / 6} r^{2} \sum \mu_{x}\left(\mathcal{O}_{r}^{+}(\gamma y, x)\right) \mu_{x}\left(\mathcal{O}_{r}^{+}(x, \gamma y)\right) e^{\delta(x, \gamma y)}+c_{1}
\end{aligned}
$$

où l'on somme sur les $\gamma \in \Gamma$ tels que

$$
(x, \gamma y) \leqslant T, \quad\left(\gamma y, \gamma^{-1} x\right) \in\left[\mathcal{C}_{1}^{+}(x, A) \times \mathcal{C}_{1}^{+}(y, B)\right] \cap[\widehat{V} \times \widehat{W}],
$$


la restriction à $\widehat{V} \times \widehat{W}$ ne faisant que contribuer à la constante $c_{1}$, puisque $\bar{V} \subset \widehat{V} \cap \partial X$ et $\bar{W} \subset \widehat{W} \cap \partial X$, et que par suite

$$
\left[\mathcal{C}_{1}^{+}(x, A) \times \mathcal{C}_{1}^{+}(y, B)\right] \backslash[\widehat{V} \times \widehat{W}]
$$

est borné dans $X^{2}$.

Nous allons maintenant établir de la même manière le pendant de cette majoration en minorant la quantité

$$
\int_{0}^{T+3 r} e^{\delta t} \sum_{\gamma \in \Gamma} m\left(K^{+} \cap g^{-t} \gamma K^{-}\right) d t .
$$

D'une part, on a généralement $d_{x}(\xi, \eta) \leqslant 1$. D'autre part, le fait que

$$
\left(\gamma y, \gamma^{-1} x\right) \in \mathcal{C}_{1}^{-}(x, A) \times \mathcal{C}_{1}^{-}(y, B)
$$

entraîne que $A \supset \mathcal{O}_{r}^{-}(x, \gamma y)$ et que $B \supset \mathcal{O}_{r}^{-}\left(y, \gamma^{-1} x\right)$, c'est-à-dire que $\gamma B \supset$ $\mathcal{O}_{r}^{-}(\gamma y, x)$. Rappelons aussi que

$$
\mathcal{L}_{r}(x, \gamma y) \supset \mathcal{O}_{r}^{-}(\gamma y, x) \times \mathcal{O}_{r}^{-}(x, \gamma y) .
$$

Enfin, pour $(\xi, \eta) \in \mathcal{L}_{r}(x, \gamma y),|s|<r / 2$, et $T>0$, on a

$$
\int_{0}^{T+3 r} e^{\delta t} \mathbb{1}_{K(\gamma y, r)}\left(g^{t+s} x_{\xi \eta}\right) d t \geqslant e^{-\varepsilon / 10} r e^{\delta(x, \gamma y)} \quad \text { si } 3 r \leqslant(x, \gamma y) \leqslant T .
$$

Il ressort de tout cela réuni la minoration $(* 2)$ suivante, quitte à augmenter $c_{1}$ :

$$
\begin{aligned}
\int_{0}^{T+3 r} e^{\delta t} \sum_{\gamma \in \Gamma} m\left(K^{+}\right. & \left.\cap g^{-t} \gamma K^{-}\right) d t \\
& \geqslant e^{-\varepsilon / 10} r^{2} \sum \mu_{x}\left(\mathcal{O}_{r}^{-}(\gamma y, x)\right) \mu_{x}\left(\mathcal{O}_{r}^{-}(x, \gamma y)\right) e^{\delta(x, \gamma y)}-c_{1}
\end{aligned}
$$

où l'on somme sur les $\gamma \in \Gamma$ tels que

$$
(x, \gamma y) \leqslant T, \quad\left(\gamma y, \gamma^{-1} x\right) \in\left[\mathcal{C}_{1}^{-}(x, A) \times \mathcal{C}_{1}^{-}(y, B)\right] \cap[\widehat{V} \times \widehat{W}] .
$$

Nous désignerons désormais par $(*)$ l'encadrement fondamental que constituent $(* 1)$ et $(* 2)$ réunis, et allons à présent examiner successivement chacun de ses membres, en commençant par les intégrales à gauche.

Puisque le flot géodésique est fortement mélangeant (théorème 3.1 ), on a, pour $t$ assez grand,

$$
e^{-\varepsilon / 3} m\left(K^{+}\right) m\left(K^{-}\right) \leqslant\left\|m_{\Gamma}\right\| \sum_{\gamma \in \Gamma} m\left(K^{+} \cap g^{-t} \gamma K^{-}\right) \leqslant e^{\varepsilon / 5} m\left(K^{+}\right) m\left(K^{-}\right) .
$$

Or, en revenant à la définition de $K^{+}=K^{+}(x, r, A)$, on voit que

$$
m\left(K^{+}\right)=r \int_{A} d \mu_{x}(\xi) \int_{\mathcal{O}_{r}(\xi, x)} d \mu_{x}(\zeta) d_{x}(\xi, \zeta)^{-2 \delta}
$$


Sachant que $d_{x}(\xi, \zeta) \geqslant e^{-r}$ dans l'intégrale ci-dessus, et tenant compte du fait que $A \subset V$, on obtient :

$$
e^{-\varepsilon / 30} r \mu_{x}(A) \mu_{x}\left(\mathcal{O}_{r}\left(\xi_{0}, x\right)\right) \leqslant m\left(K^{+}\right) \leqslant e^{\varepsilon / 10} r \mu_{x}(A) \mu_{x}\left(\mathcal{O}_{r}\left(\xi_{0}, x\right)\right) .
$$

De même,

$$
e^{-\varepsilon / 30} r \mu_{y}(B) \mu_{y}\left(\mathcal{O}_{r}\left(\eta_{0}, y\right)\right) \leqslant m\left(K^{-}\right) \leqslant e^{\varepsilon / 10} r \mu_{y}(B) \mu_{y}\left(\mathcal{O}_{r}\left(\eta_{0}, y\right)\right) .
$$

De ce qui précède, il vient, avec une constante $c_{2}$ indépendante de $T$, en notant $M=r^{2} \mu_{x}\left(\mathcal{O}_{r}\left(\xi_{0}, x\right)\right) \mu_{y}\left(\mathcal{O}_{r}\left(\eta_{0}, y\right)\right)$ :

$$
\begin{aligned}
& \delta\left\|m_{\Gamma}\right\| \int_{0}^{T-3 r} e^{\delta t} \sum_{\gamma \in \Gamma} m\left(K^{+} \cap g^{-t} \gamma K^{-}\right) d t \geqslant e^{-\varepsilon / 2} e^{\delta T} M \mu_{x}(A) \mu_{y}(B)-c_{2}, \\
& \delta\left\|m_{\Gamma}\right\| \int_{0}^{T+3 r} e^{\delta t} \sum_{\gamma \in \Gamma} m\left(K^{+} \cap g^{-t} \gamma K^{-}\right) d t \leqslant e^{\varepsilon / 2} e^{\delta T} M \mu_{x}(A) \mu_{y}(B)+c_{2} .
\end{aligned}
$$

Passons maintenant aux sommes à droite dans l'encadrement $(*)$.

Par le lemme 1.2 légèrement modifié pour la circonstance, on a

$$
\begin{aligned}
\mu_{y}\left(\mathcal{O}_{r}^{ \pm}\left(\gamma^{-1} x, y\right)\right)=\mu_{\gamma y}\left(\mathcal{O}_{r}^{ \pm}(x, \gamma y)\right) & \leqslant \mu_{x}\left(\mathcal{O}_{r}^{ \pm}(x, \gamma y)\right) e^{\delta(x, \gamma y)} \\
& \leqslant e^{\delta 3 r} \mu_{\gamma y}\left(\mathcal{O}_{r}^{ \pm}(x, \gamma y)\right) \leqslant e^{\varepsilon / 10} \mu_{y}\left(\mathcal{O}_{r}^{ \pm}\left(\gamma^{-1} x, y\right)\right) .
\end{aligned}
$$

Lorsque $\left(\gamma y, \gamma^{-1} x\right) \in \widehat{V} \times \widehat{W}$, il en résulte que

$$
\begin{aligned}
\mu_{x}\left(\mathcal{O}_{r}^{+}(\gamma y, x)\right) \mu_{x}\left(\mathcal{O}_{r}^{+}(x, \gamma y)\right) e^{\delta(x, \gamma y)} & \leqslant e^{\varepsilon / 10} \mu_{x}\left(\mathcal{O}_{r}^{+}(\gamma y, x)\right) \mu_{y}\left(\mathcal{O}_{r}^{+}\left(\gamma^{-1} x, y\right)\right) \\
& \leqslant e^{\varepsilon / 6} \mu_{x}\left(\mathcal{O}_{r}\left(\xi_{0}, x\right)\right) \mu_{y}\left(\mathcal{O}_{r}\left(\eta_{0}, y\right)\right),
\end{aligned}
$$

et de même

$$
\mu_{x}\left(\mathcal{O}_{r}^{-}(\gamma y, x)\right) \mu_{x}\left(\mathcal{O}_{r}^{-}(x, \gamma y)\right) e^{\delta(x, \gamma y)} \geqslant e^{-\varepsilon / 15} \mu_{x}\left(\mathcal{O}_{r}\left(\xi_{0}, x\right)\right) \mu_{y}\left(\mathcal{O}_{r}\left(\eta_{0}, y\right)\right) .
$$

Grâce au fait que $\mu_{x}\left(\mathcal{O}_{r}\left(\xi_{0}, x\right)\right) \mu_{y}\left(\mathcal{O}_{r}\left(\eta_{0}, y\right)\right)>0$ (voir plus haut), l'encadrement (*) et ceux qui suivent mènent au suivant :

$$
\begin{gathered}
e^{-\varepsilon / 2} \mu_{x}(A) \mu_{y}(B) \leqslant e^{\varepsilon / 3} \nu_{x, y}^{T}\left(\mathcal{C}_{1}^{+}(x, A) \times \mathcal{C}_{1}^{+}(y, B)\right)+c_{3} e^{-\delta T} \\
\text { et } e^{\varepsilon / 2} \mu_{x}(A) \mu_{y}(B) \geqslant e^{-\varepsilon / 6} \nu_{x, y}^{T}\left(\mathcal{C}_{1}^{-}(x, A) \times \mathcal{C}_{1}^{-}(y, B)\right)-c_{3} e^{-\delta T},
\end{gathered}
$$

avec $c_{3}$ indépendante de $T$. On aboutit ainsi à la conclusion désirée.

Deuxième étape. - Soient $x, y \in X$. Soit $\varepsilon>0$. Pour tout $\left(\xi_{0}, \eta_{0}\right) \in \partial X \times \partial X$, il existe $r>0$ et des voisinages $V$ et $W$ respectivement de $\xi_{0}$ et $\eta_{0}$ dans $\partial X$, tels que pour tous boréliens $A \subset V$ et $B \subset W$, on a, quand $t \rightarrow+\infty$,

$$
\begin{aligned}
\limsup \nu_{x, y}^{t}\left(\mathcal{C}_{r}^{-}(x, A) \times \mathcal{C}_{r}^{-}(y, B)\right) & \leqslant e^{\varepsilon} \mu_{x}(A) \mu_{y}(B) \\
\text { et } \quad \liminf \nu_{x, y}^{t}\left(\mathcal{C}_{r}^{+}(x, A) \times \mathcal{C}_{r}^{+}(y, B)\right) & \geqslant e^{-\varepsilon} \mu_{x}(A) \mu_{y}(B) .
\end{aligned}
$$


Démonstration. - Soient $\xi_{0}, \eta_{0} \in \partial X$. Choisissons $\zeta_{0} \in \Lambda(\Gamma)$, puis $x_{0} \in\left(\xi_{0} \zeta_{0}\right)$ et $y_{0} \in\left(\eta_{0} \zeta_{0}\right)$. On dispose alors de voisinages $V_{0}$ et $W_{0}$ respectivement de $\xi_{0}$ et $\eta_{0}$ tels que l'énoncé de la première étape soit valide pour $x_{0}$ et $y_{0}$ à la place de $x$ et $y, V_{0}$ et $W_{0}$ à celle de $V$ et $W$, et enfin $\varepsilon / 3$ à celle de $\varepsilon$. Soient alors $\widehat{V_{0}}$ et $\widehat{W_{0}}$ deux ouverts de $\bar{X}$ tels que $\widehat{V_{0}} \cap \partial X \subset V_{0}, \widehat{W_{0}} \cap \partial X \subset W_{0}$, et pour tous $a \in \widehat{V_{0}}$ et $b \in \widehat{W}_{0}$,

$$
\left|\left(x_{0}, a\right)-(x, a)-\beta_{\xi_{0}}\left(x_{0}, x\right)\right|<\frac{\varepsilon}{6 \delta} \quad \text { et } \quad\left|\left(y_{0}, b\right)-(y, b)-\beta_{\eta_{0}}\left(y_{0}, y\right)\right|<\frac{\varepsilon}{6 \delta}
$$

(si $a=\xi \in \partial X$, la quantité $\left(x_{0}, a\right)-(x, a)$ est à prendre au sens de $\beta_{\xi}\left(x_{0}, x\right)$, idem pour $b$ ).

Prenons alors deux ouverts $V$ et $W$ voisinages respectifs de $\xi_{0}$ et de $\eta_{0}$ dans $\partial X$, tels que $\bar{V} \subset \widehat{V_{0}} \cap \partial X$ et $\bar{W} \subset \widehat{W_{0}} \cap \partial X$, et $r=1+\max \left\{\left(x, x_{0}\right),\left(y, y_{0}\right)\right\}$. Considérons $A \subset V$ et $B \subset W$. Le fond de la démonstration est que l'on peut rapporter l'orbite de $y$ vue de $x$ à celle de $y_{0}$ vue de $x_{0}$ de la façon suivante.

En effet, si $\left(\gamma y, \gamma^{-1} x\right) \in \mathcal{C}_{r}^{-}(x, A) \times \mathcal{C}_{r}^{-}(y, B)$, on peut facilement vérifier qu'alors $\left(\gamma y_{0}, \gamma^{-1} x_{0}\right) \in \mathcal{C}_{1}^{-}\left(x_{0}, A\right) \times \mathcal{C}_{1}^{-}\left(y_{0}, B\right)$, grâce au choix de $r$. D'autre part, si $(x, \gamma y) \leqslant t$ et $\left(\gamma y, \gamma^{-1} x\right) \in \widehat{V_{-r}} \times \widehat{W_{0}}$, en notant $\widehat{V_{-r}}=\left\{z \in X \mid B(z, r) \subset \widehat{V_{0}}\right\}$, alors $\gamma y_{0} \in \widehat{V_{0}}$ et $\gamma^{-1} x \in \widehat{W}_{0}$, d'où il résulte successivement que

$$
\begin{aligned}
\left(x_{0}, \gamma y_{0}\right) & \leqslant\left(x, \gamma y_{0}\right)+\beta_{\xi_{0}}\left(x_{0}, x\right)+\frac{\varepsilon}{6 \delta}=\left(y_{0}, \gamma^{-1} x\right)+\beta_{\xi_{0}}\left(x_{0}, x\right)+\frac{\varepsilon}{6 \delta} \\
& \leqslant\left(y, \gamma^{-1} x\right)+\beta_{\eta_{0}}\left(y_{0}, y\right)+\beta_{\xi_{0}}\left(x_{0}, x\right)+\frac{\varepsilon}{3 \delta} \\
& \leqslant t+\beta_{\xi_{0}}\left(x_{0}, x\right)+\beta_{\eta_{0}}\left(y_{0}, y\right)+\frac{\varepsilon}{3 \delta} .
\end{aligned}
$$

Comme $\left[\mathcal{C}_{r}^{-}\left(x, A^{+}\right) \times \mathcal{C}_{r}^{-}\left(y, B^{+}\right)\right] \backslash\left[\widehat{V_{-r}} \times \widehat{W_{0}}\right]$ est borné dans $X^{2}$, on en déduit que la limite supérieure (quand $t \rightarrow+\infty)$ de $\nu_{x, y}^{t}\left(\mathcal{C}_{r}^{-}(x, A) \times \mathcal{C}_{r}^{-}(y, B)\right)$ est plus petite que

$$
e^{\varepsilon / 3} e^{\delta \beta_{\xi_{0}}\left(x_{0}, x\right)+\delta \beta_{\eta_{0}}\left(y_{0}, y\right)} \lim \sup \nu_{x_{0}, y_{0}}^{t+\beta_{\xi_{0}}\left(x_{0}, x\right)+\beta_{\eta_{0}}\left(y_{0}, y\right)+\varepsilon / 3 \delta}\left(\mathcal{C}_{1}^{-}\left(x_{0}, A\right) \times \mathcal{C}_{1}^{-}\left(y_{0}, B\right)\right) .
$$

En appliquant la première étape, il en résulte que

$$
\limsup \nu_{x, y}^{t}\left(\mathcal{C}_{r}^{-}(x, A) \times \mathcal{C}_{r}^{-}(y, B)\right) \leqslant e^{2 \varepsilon / 3} e^{\delta \beta_{\xi_{0}}\left(x_{0}, x\right)+\delta \beta_{\eta_{0}}\left(y_{0}, y\right)} \mu_{x_{0}}(A) \mu_{y_{0}}(B) .
$$

Or $e^{\delta \beta_{\xi_{0}}\left(x_{0}, x\right)} \mu_{x_{0}} \leqslant e^{\varepsilon / 6} \mu_{x}$ en restriction à $V$, ainsi que $e^{\delta \beta_{\eta_{0}}\left(y_{0}, y\right)} \mu_{y_{0}} \leqslant e^{\varepsilon / 6} \mu_{y}$ sur $W$, d'où

$$
\limsup \nu_{x, y}^{t}\left(\mathcal{C}_{r}^{-}(x, A) \times \mathcal{C}_{r}^{-}(y, B)\right) \leqslant e^{\varepsilon} \mu_{x}(A) \mu_{y}(B),
$$

ce qu'il fallait montrer.

L’inégalité complémentaire se montre de façon entièrement analogue.

Troisième étape : conclusion. - Soient $x, y \in X$. Conservons $\varepsilon>0$, les deux ouverts $V$ et $W$ et $r$ de la seconde étape, prenons $\widehat{V}$ et $\widehat{W}$ deux ouverts de $\bar{X}$ tels que $\widehat{V} \cap \partial X=V$ et $\widehat{W} \cap \partial X=W$, et considérons deux boréliens $\mathcal{A}$ et $\mathcal{B}$ de $\bar{X}$ tels que 
$\overline{\mathcal{A}} \subset \widehat{V}$ et $\overline{\mathcal{B}} \subset \widehat{W}$, et tels que $\left(\mu_{x} \otimes \mu_{y}\right)(\partial(\mathcal{A} \times \mathcal{B}))=0$, ce qui revient à dire que $\mu_{x}(\partial \mathcal{A}) \mu_{y}(\overline{\mathcal{B}})=0=\mu_{x}(\overline{\mathcal{A}}) \mu_{y}(\partial \mathcal{B})$.

Soit $\alpha>0$. Prenons des ouverts $A^{+}$et $B^{+}$ainsi que des compacts $A^{-}$et $B^{-}$dans $\partial X$ tels que :

$$
\begin{gathered}
A^{-} \subset \mathcal{A}^{o} \cap \partial X \subset \overline{\mathcal{A}} \cap \partial X \subset A^{+} \subset V, \\
B^{-} \subset \mathcal{B}^{o} \cap \partial X \subset \overline{\mathcal{B}} \cap \partial X \subset B^{+} \subset W \\
\mu_{x}\left(\mathcal{A}^{o} \backslash A^{-}\right)<\alpha, \quad \mu_{x}\left(A^{+} \backslash \overline{\mathcal{A}}\right)<\alpha, \quad \mu_{y}\left(\mathcal{B}^{o} \backslash B^{-}\right)<\alpha, \quad \mu_{y}\left(B^{+} \backslash \overline{\mathcal{B}}\right)<\alpha .
\end{gathered}
$$

Il est aisé de voir que les ensembles

$$
[\overline{\mathcal{A}} \times \overline{\mathcal{B}}] \backslash\left[\mathcal{C}_{r}^{-}\left(x, A^{+}\right) \times \mathcal{C}_{r}^{-}\left(y, B^{+}\right)\right] \quad \text { et } \quad\left[\mathcal{C}_{r}^{+}\left(x, A^{-}\right) \times \mathcal{C}_{r}^{+}\left(y, B^{-}\right)\right] \backslash\left[\mathcal{A}^{o} \times \mathcal{B}^{o}\right]
$$

sont relativement compacts dans $X \times X$. Il résulte de cela puis de la seconde étape que

$$
\begin{aligned}
\limsup \nu_{x, y}^{t}(\mathcal{A} \times \mathcal{B}) & \leqslant \limsup \nu_{x, y}^{t}\left(\mathcal{C}_{r}^{-}\left(x, A^{+}\right) \times \mathcal{C}_{r}^{-}\left(y, B^{+}\right)\right) \\
& \leqslant e^{\varepsilon} \mu_{x}\left(A^{+}\right) \mu_{y}\left(B^{+}\right) \\
& \leqslant e^{\varepsilon} \mu_{x}(\overline{\mathcal{A}}) \mu_{y}(\overline{\mathcal{B}})+\alpha e^{\varepsilon}\left(\left\|\mu_{x}\right\|+\left\|\mu_{y}\right\|\right) \\
& \leqslant e^{\varepsilon} \mu_{x}(\mathcal{A}) \mu_{y}(\mathcal{B})+\alpha e^{\varepsilon}\left(\left\|\mu_{x}\right\|+\left\|\mu_{y}\right\|\right)
\end{aligned}
$$

puisque $\left(\mu_{x} \otimes \mu_{y}\right)(\partial(\mathcal{A} \times \mathcal{B}))=0$. Comme $\alpha>0$ était arbitrairement petit, on obtient en définitive que

$$
\limsup \nu_{x, y}^{t}(\mathcal{A} \times \mathcal{B}) \leqslant e^{\varepsilon} \mu_{x}(\mathcal{A}) \mu_{y}(\mathcal{B}) .
$$

De façon analogue, vient l'inégalité complémentaire :

$$
\liminf \nu_{x, y}^{t}(\mathcal{A} \times \mathcal{B}) \geqslant e^{-\varepsilon} \mu_{x}(\mathcal{A}) \mu_{y}(\mathcal{B}) .
$$

On en déduit que pour toute fonction $h$ positive, continue et à support dans $\widehat{V} \times \widehat{W}$,

$$
e^{-\varepsilon} \int \varphi d \mu_{x} \otimes \mu_{y} \leqslant \liminf \int \varphi d \nu_{x, y}^{t} \leqslant \lim \sup \int \varphi d \nu_{x, y}^{t} \leqslant e^{\varepsilon} \int \varphi d \mu_{x} \otimes \mu_{y} .
$$

Comme on peut recouvrir le compact $\partial X \times \partial X$ par un nombre fini d'ouverts du type $V \times W$, et par extension $\bar{X} \times \bar{X}$ par des ouverts $\widehat{V} \times \widehat{W}$ comme ci-dessus, on voit, à l'aide d'une partition de l'unité subordonnée à un tel recouvrement, que l'encadrement ci-dessus reste valide pour toute fonction continue positive sur $\bar{X} \times \bar{X}$. Il ne reste plus qu'à faire tendre $\varepsilon$ vers 0 pour clore la démonstration.

En complément, nous proposons un résultat d'équidistribution des orbites des points fixes paraboliques, que nous tirerons à nouveau du mélange du flot géodésique, mais ici sous la forme du corollaire 3.2. Le cas où $X$ est l'espace hyperbolique de dimension 2 ou 3 a déjà été traité dans [Cos], où l'on en verra des liens avec l'arithmétique, ce qui était également la motivation de $[\mathbf{B e}-\mathbf{H}-\mathbf{P}]$. Ce résultat est d'ailleurs utilisé dans [H-P2]. 
THÉORÈmE 4.2. - Soit $m_{\Gamma}$ une mesure de Bowen-Margulis-Sullivan associée à une densité $\mu$ conforme de dimension $\delta=\delta(\Gamma)$ et invariante par $\Gamma$. Soient $p$ un point parabolique de $\Gamma$ (s'il en existe) et $\Pi$ le sous-groupe parabolique maximal de $\Gamma$ stabilisant p. Alors, pour toute horosphère $H$ dans $X$ basée au point $p$, quand $t$ tend vers $+\infty$,

$$
e^{-\delta t} \sum_{\substack{\gamma \in \Gamma / \Pi \\ 0 \leqslant(H, \gamma H) \leqslant t}} \mathcal{D}_{\gamma p} \rightarrow \frac{P_{H}^{*} \mu_{H}(F)}{\delta\left\|m_{\Gamma}\right\|} P_{H}^{*} \mu_{H} \quad \text { faiblement dans } C_{c}(\partial X \backslash\{p\})^{*}
$$

où $\Gamma / \Pi$ désigne un système de représentants des classes à gauche de $\Gamma$ selon $\Pi$, et $F$ un quelconque domaine fondamental pour l'action de $\Pi$ sur $\partial X$, de bord $\mu_{o^{-}}$ négligeable. Cela dès que l'expression de la limite ci-dessus a un sens, c'est-à-dire lorsque $P_{H}^{*} \mu_{H}(F)$ et $\left\|m_{\Gamma}\right\|$ ne sont pas simultanément infinis. (L'expression $(H, \gamma H)$ désigne la distance de Hausdorff.)

Corollaire 1. - Soit h un quelconque point de H. Alors,

$$
e^{-\delta t} \sum_{\substack{\gamma \in \Gamma / \Pi \\ 0 \leqslant(h, \gamma H) \leqslant t}} \mathcal{D}_{\gamma p} \rightarrow \frac{P_{H}^{*} \mu_{H}(F)}{\delta\left\|m_{\Gamma}\right\|} \mu_{h} \quad \text { faiblement dans } C(\partial X)^{*} .
$$

Corollaire 2. - Supposons en outre que le point parabolique p soit borné. Alors

$$
\#\{\gamma \in \Pi \backslash \Gamma / \Pi \mid 0 \leqslant(H, \gamma H) \leqslant t\} \sim \frac{P_{H}^{*} \mu_{H}(F)^{2}}{\delta\left\|m_{\Gamma}\right\|} e^{\delta t},
$$

où $\Pi \backslash \Gamma / \Pi$ désigne un système de représentants des doubles classes de $\Gamma$ selon $\Pi$.

Démonstration. - Soit $H$ une horosphère basée en $p$. Notons $\nu^{T}$ la mesure de l'énoncé indexée ici par $T>0$ plutôt que par $t$. L'horosphère $H$ détermine une horosphère stable (resp. instable) que nous noterons $H^{-}$(resp. $H^{+}$).

Fixons $\varepsilon>0$ arbitrairement petit. Étant donné un quelconque point $\xi \in \partial X \backslash\{p\}$, nommons $u=P_{H^{-}} \xi$, et prenons $r_{1}, r_{2}, r_{3}>0$ assez petits afin que la conclusion $\mathrm{du}$ lemme 1.15 soit valide pour la mesure $m$, et que pour tout $v \in B^{-}\left(u, r_{1}\right)$ l'on ait (grâce au lemme 1.13) :

$$
e^{-\varepsilon / 8} \beta \leqslant \mu_{H^{+}(v)}\left(P_{H^{+}(v)} P_{H^{+}(u)}^{-1}\left(B^{+}\left(u, r_{2}\right)\right)\right) \leqslant e^{\varepsilon / 8} \beta
$$

où $\beta=\mu_{H^{+}(u)}\left(B^{+}\left(u, r_{2}\right)\right)$. Nous pouvons supposer en outre que $0<r_{3}<\varepsilon / 8 \delta$ et que $\mu_{H^{+}}\left(\partial B^{+}\left(u, r_{2}\right)\right)=0$. Considérons alors un quelconque borélien $E$ contenu dans le voisinage $P_{H^{-}}^{-1}\left(B^{-}\left(u, r_{1}\right)\right)$ de $\xi$ et de bord $\mu_{o}$-négligeable. Nous nous proposons d'estimer $\nu^{T}(E)$ asymptotiquement quand $T \rightarrow+\infty$.

Désignons par $C$ la cellule $C\left(E^{-}, B^{+}\left(u, r_{2}\right), r_{3}\right)$ où $E^{-}=P_{H^{-}} E$. On observera que $m(\partial C)=0$ par construction de $C$. Notons $F^{+}=P_{H^{+}} F$, où $F$ est comme dans 
l'énoncé. En un mot, notre méthode consiste à rapporter à $\nu^{T}(E)$ la quantité

$$
\int_{0}^{T} e^{\delta t} \sum_{\gamma \in \Gamma} \mu_{H^{+}}\left(F^{+} \cap g^{-t} \gamma C\right) d t
$$

dont on connaît par ailleurs le comportement asymptotique grâce au corollaire 3.2.

En usant de faits élémentaires, on voit que

$$
e^{\delta t} \sum_{\gamma \in \Gamma} \mu_{H^{+}}\left(F^{+} \cap g^{-t} \gamma C\right)=\sum_{\gamma \in \Gamma} \mu_{g^{t} \gamma H^{+}}\left(g^{t} \gamma F^{+} \cap C\right)=\sum_{\gamma \in \Gamma / \Pi} \mu_{g^{t} \gamma H^{+}}\left(g^{t} \gamma H^{+} \cap C\right) \text {. }
$$

Mais si $g^{t} \gamma H^{+} \cap C \neq \varnothing$, alors

$$
g^{t} \gamma H^{+} \cap C=g^{r} P_{H^{+}(v)} P_{H^{+}(u)}^{-1}\left(B^{+}\left(u, r_{2}\right)\right)
$$

pour un $v \in E^{-} \subset B^{-}\left(u, r_{1}\right)$ et un $\left.r \in\right]-r_{3}, r_{3}[$, et par conséquent

$$
e^{-\varepsilon / 4} \beta \leqslant \mu_{g^{t} \gamma H^{+}}\left(g^{t} \gamma H^{+} \cap C\right) \leqslant e^{\varepsilon / 4} \beta \text {. }
$$

On obtient donc l'encadrement suivant, où $n(t)=\#\left\{\gamma \in \Gamma / \Pi \mid g^{t} \gamma H^{+} \cap C \neq \varnothing\right\}$ :

$$
e^{-\varepsilon / 4} \beta n(t) \leqslant e^{\delta t} \sum_{\gamma \in \Gamma} \mu_{H^{+}}\left(F^{+} \cap g^{-t} \gamma C\right) \leqslant e^{\varepsilon / 4} \beta n(t) .
$$

En poursuivant l'analyse précédente, on voit que $g^{t} \gamma H^{+} \cap C$ n'est pas vide si et seulement si $g^{t} \gamma H^{+}$rencontre $\bigcup_{|r|<r_{3}} g^{r} E^{-}$, ce qui équivaut encore aux faits réunis que $\gamma p \in E$ et que $t-r_{3} \leqslant(H, \gamma H) \leqslant t+r_{3}$ (car $E^{-}$est contenu dans $H^{-}$qui comme $H^{+}$se projette sur $\left.H\right)$. On en déduit aisément que $\int_{r_{3}}^{T-r_{3}} n(t) d t$ est majorée par $2 r_{3}$ fois le nombre de $\gamma \in \Gamma / \Pi$ tels que $\gamma p \in E$ et $0 \leqslant(H, \gamma H) \leqslant T$, d'où

$$
\int_{r_{3}}^{T-r_{3}} e^{\delta t} \sum_{\gamma \in \Gamma} \mu_{H^{+}}\left(F^{+} \cap g^{-t} \gamma C\right) d t \leqslant e^{\varepsilon / 4} \beta 2 r_{3} e^{\delta T} \nu^{T}(E) .
$$

De même a-t-on

$$
\int_{-r_{3}}^{T+r_{3}} e^{\delta t} \sum_{\gamma \in \Gamma} \mu_{H^{+}}\left(F^{+} \cap g^{-t} \gamma C\right) d t \geqslant e^{-\varepsilon / 4} \beta 2 r_{3} e^{\delta T} \nu^{T}(E) .
$$

D'autre part, le corollaire 3.2 nous donne

$$
\sum_{\gamma \in \Gamma} \mu_{H^{+}}\left(F^{+} \cap g^{-t} \gamma C\right) \longrightarrow \mu_{H^{+}}\left(F^{+}\right) \frac{m(C)}{\left\|m_{\Gamma}\right\|}
$$

quand $t \rightarrow+\infty$, d'où, lorsque $T \rightarrow+\infty$,

$$
\int_{0}^{T} e^{\delta t} \sum_{\gamma \in \Gamma} \mu_{H^{+}}\left(F^{+} \cap g^{-t} \gamma C\right) d t \sim e^{\delta T} \frac{\mu_{H^{+}}\left(F^{+}\right)}{\left\|m_{\Gamma}\right\|} m(C) .
$$

Enfin, grâce au lemme 1.15, on a

$$
e^{-\varepsilon / 4} \beta 2 r_{3} \mu_{H^{-}}\left(E^{-}\right) \leqslant m(C) \leqslant e^{\varepsilon / 4} \beta 2 r_{3} \mu_{H^{-}}\left(E^{-}\right) .
$$


Notons que $\mu_{H^{-}}\left(E^{-}\right)=P_{H}^{*} \mu_{H}(E)$ et que $\mu_{H^{+}}\left(F^{+}\right)=P_{H}^{*} \mu_{H}(F)$, et de tout ce qui précède, nous obtenons en définitive que, pour $T$ assez grand,

$$
e^{-\varepsilon} \frac{P_{H}^{*} \mu_{H}(F)}{\delta\left\|m_{\Gamma}\right\|} P_{H}^{*} \mu_{H}(E) \leqslant \nu^{T}(E) \geqslant e^{\varepsilon} \frac{P_{H}^{*} \mu_{H}(F)}{\delta\left\|m_{\Gamma}\right\|} P_{H}^{*} \mu_{H}(E),
$$

ce pour tout borélien $E$ de bord $\mu_{o}$-négligeable contenu dans un voisinage de $\xi$, qui était quelconque dans $\partial X \backslash\{p\}$. De là, on étend aisément la validité de cet encadrement pour tout borélien relativement compact dans $\partial X \backslash\{p\}$ et de bord $\mu_{o^{-}}$ négligeable, et l'on fait tendre $\varepsilon$ vers 0 pour terminer la démonstration du théorème.

Le corollaire 1 s'obtient aisément en renormalisant corrélativement les mesures $\nu^{t}$ et $d\left(P_{H}^{*} \mu_{H}\right)(\xi)=e^{\delta \beta_{\xi}\left(o, P_{H} \xi\right)} d \mu_{o}(\xi)$ au voisinage de chaque point de $\partial X \backslash\{p\}$. On conclut en tenant compte du fait que $\mu_{h}(\{p\})=0$ quand $\left\|m_{\Gamma}\right\|<\infty$.

Le corollaire 2 n'exprime rien de plus que la convergence de $\nu^{t}(F)$ vers la valeur que donne le théorème, sachant que l'on peut supposer $F \cap \Lambda(\Gamma)$ relativement compact, puisque $p$ est borné. 


\section{CHAPITRE 5}

\section{ÉQUIDISTRIBUTION ASYMPTOTIQUE DES GÉODÉSIQUES FERMÉES PRIMITIVES}

Dans ce chapitre, nous faisons correspondre au théorème 4.1 un résultat d'équidistribution des géodésiques fermées primitives sur $S X / \Gamma$ (théorème 5.2). Le problème du dénombrement asymptotique des géodésiques fermées primitives sera plus particulièrement examiné dans le théorème 5.2. Rappelons que depuis le chapitre 3 , il est supposé que le spectre des longueurs du groupe $\Gamma$ n'est pas arithmétique.

Nous ne nous étendrons pas plus sur la bibliographie du sujet que dans le chapitre précédent; nous indiquerons seulement que le théorème 5.1 .1 a déjà été obtenu pour les variétés convexe-cocompactes (voir $[\mathbf{L}],[\mathbf{P o l}-\mathbf{S}]$ ), et certaines variétés géométriquement finies avec des pointes (voir [Dal-P], où l'on trouvera en outre une liste de références). Pour ce qui est des méthodes et de la portée des résultats, on pourrait répéter les mêmes commentaires que ceux qui accompagnent le théorème 4.1, et nous y renvoyons le lecteur. Nous ajouterons seulement que les deux problèmes d'équidistribution que traitent les théorèmes 4.1 d'une part, et 5.1 d'autre part, ont été d'ordinaire abordés plus ou moins séparément, tandis qu'ils sont ici mis en relation : nous verrons en effet que l'énoncé 5.1 découle de 4.1, après simple examen et transformation des mesures orbitales apparues dans ce dernier; mais l'on aurait tout aussi bien pu montrer que ces deux énoncés sont en fait exactement équivalents.

Ce que nous entendons par géodésique fermée sur $S X / \Gamma$, notamment en présence de torsion dans $\Gamma$, a déjà été précisé à la fin du paragraphe $1 \mathrm{C}$ : la projection canonique de $S X$ sur $S X / \Gamma$ envoie les axes orientés des isométries hyperboliques dans $\Gamma$ sur ce que nous appelons les géodésiques fermées de $S X / \Gamma$. Cette projection induit une correspondance biunivoque entre classes de conjugaison d'isométries hyperboliques primitives de $\Gamma$ et géodésiques fermées primitives de $S X / \Gamma$, une isométrie hyperbolique $\gamma \in \Gamma$ étant dite primitive lorsqu'il n'existe pas de $\phi \in \Gamma$ ni d'entier $n>1$ tels que $\gamma=\phi^{n}$. Nous noterons $\mathcal{G}_{\Gamma}(\ell)$ l'ensemble des géodésiques fermées primitives dans $S X / \Gamma$ de longueur au plus $\ell$. Pour une géodésique fermée primitive $g$, on désignera par $\mathcal{D}_{g}$ la mesure de Lebesgue le long de $g$ normalisée; la probabilité $\mathcal{D}_{g}$ est souvent appelée 
masse de Dirac en $g$. La notation $C_{c}(S X / \Gamma)^{*}$ signifie le dual faible-* de l'espace des fonctions continues à support compact dans $S X / \Gamma$. Désormais, $\delta$ désignera $\delta(\Gamma)$.

ThÉORÈme 5.1.1. - Supposons que $\Gamma$ admette une mesure de Bowen-MargulisSullivan $m_{\Gamma}$ finie. Alors, quand $\ell \rightarrow+\infty$,

$$
\delta \ell e^{-\delta \ell} \sum_{g \in \mathcal{G}_{\Gamma}(\ell)} \mathcal{D}_{g} \rightarrow \frac{m_{\Gamma}}{\left\|m_{\Gamma}\right\|} \quad \text { faiblement dans } C_{c}(S X / \Gamma)^{*} .
$$

ThÉORÈme 5.1.2. - Supposons que $\Gamma$ n'admette pas de mesure de Bowen-MargulisSullivan finie. Alors, quand $\ell \rightarrow+\infty$,

$$
\delta \ell e^{-\delta \ell} \sum_{g \in \mathcal{G}_{\Gamma}(\ell)} \mathcal{D}_{g} \rightarrow 0 \quad \text { faiblement dans } C_{c}(S X / \Gamma)^{*} .
$$

Démonstration. - Nous nous contenterons de détailler la seule preuve du théorème 5.1.1; l'énoncé complémentaire s'obtient de la même manière, en suivant le cours de la présente preuve (on utilisera le théorème 4.1.2 à la place du théorème 4.1.1), mais avec bien des simplifications, vu la relative grossièreté de l'énoncé dans cette situation.

Nous nous appuierons sur un lemme de nature géométrique, très clair dans le disque hyperbolique, mais qui s'étend dans notre cadre général par comparaison à ce dernier.

Lemme. - Soient $x \in X$ et $r>0$ fixés. Soit $\varepsilon>0$. Il existe $t_{0}=t_{0}(x, r, \varepsilon)>0$ tel que pour toute isométrie $\phi$ de $X$, si $(x, \phi x)>t_{0}$ et si la géodésique $\left(\phi^{-1} x \phi x\right)$ rencontre $\bar{B}(x, r)$, alors $\phi$ est hyperbolique, et $e^{-\left\langle\phi^{ \pm} x, \phi^{ \pm}\right\rangle_{x}}<\varepsilon$ où $\phi^{-}$(resp. $\left.\phi^{+}\right)$désigne le point fixe répulsif (resp. attractif) de $\phi$ (cela n'est rien d'autre qu'une façon d'exprimer que $\phi^{ \pm} x$ sont arbitrairement proches de $\phi^{ \pm}$quand $(x, \phi x)$ devient grand).

Démonstration du lemme. - Supposons que $\left(\phi^{-1} x \phi x\right)$ rencontre $\bar{B}(x, r)$. Si $\phi$ est parabolique (resp. elliptique), alors les points $x, \phi^{-1} x$ et $\phi x$ sont situés sur une même horosphère (resp. un cercle, dont la conformation en s'agrandissant tend vers celle de l'horosphère), et par suite $(x, \phi x)$ ne saurait être trop grande. Si au contraire cette distance est supérieure à un certain $t_{0}$, l'isométrie $\phi$ est nécessairement hyperbolique; soit alors $a$ (resp. $p$ ) le «projeté orthogonal» de $x$ sur l'axe de $\phi\left(\right.$ resp. sur $\left.\left(\phi^{-1} x \phi x\right)\right)$; avec $t_{0}$ assez grand, le point $p$ se trouve entre $\phi^{-1} x$ et $\phi x$; envoyons les triangles $\left(\phi^{-1} x, \phi^{-1} a, \phi x\right)$ et $\left(\phi^{-1} a, \phi x, \phi a\right)$ sur des triangles de comparaison adjacents suivant le côté $\left[\overline{\phi^{-1} a}, \overline{\phi x}\right]$; comme $\left(\overline{\phi^{-1} a}, \overline{\phi^{-1} x}\right)=(\overline{\phi a}, \overline{\phi x})=(a, x)$, on vérifie, à l'aide de la géométrie hyperbolique, que $(\bar{a}, \bar{p}) \leqslant \max \{1,(a, x)-r-1\}$ pourvu que $t_{0}$ soit assez grand; il en résulte que $(a, x)$ (qui est plus petit que $(a, p)+r)$ est borné $(\operatorname{par} r+2)$, et le résultat voulu s'ensuit facilement.

Notons $\mathcal{E}^{\ell}$ la mesure sur $S X$ au-dessus de celle sur $S X / \Gamma$ apparaissant dans l'énoncé du théorème. Il nous faut prouver que $\mathcal{E}^{\ell} \rightarrow m /\left\|m_{\Gamma}\right\|$ faiblement dans $C_{c}(S X)^{*}$ quand 
$\ell \rightarrow+\infty$. Soient $\mu$ la densité conforme de dimension $\delta$ et invariante par $\Gamma$, à laquelle $m$ est associée, et $\nu$ la mesure sur $\partial^{2} X$ donnée par

$$
d \nu(\xi, \eta)=\frac{d \mu_{x}(\xi) d \mu_{x}(\eta)}{d_{x}(\xi, \eta)^{2 \delta}}
$$

(indépendante du choix de $x$ ). Rappelons que par définition, $m=\nu \otimes d s$ sur $\partial^{2} X \times \mathbb{R}=$ $S X$. Nous allons d'abord tirer du théorème 4.1 une mesure $\nu_{x, 1}^{t}$ convergeant vaguement vers $\nu$ quand $t \rightarrow+\infty$, puis modifier $\nu_{x, 1}^{t}$ successivement en $\nu_{x, 2}^{t}$ puis $\nu_{x, 3}^{t}$, de façon que $\nu_{x, 3}^{t}$ soit portée par les paires d'extrémités des axes des éléments hyperboliques de $\Gamma$ et qu'elle approche localement $\nu$. En prenant le produit de $\left\|m_{\Gamma}\right\|^{-1} \nu_{x, 3}^{t}$ par la mesure de Lebesgue sur $\mathbb{R}$, on obtiendra alors une mesure $\mathcal{M}_{x, 3}^{t}$ approchant $\left\|m_{\Gamma}\right\|^{-1} m$ localement (à savoir autour de la fibre de $x$ dans $S X$ ). Pour terminer, on rapportera $\mathcal{M}_{x, 3}^{t}$, après dernière modification en $\mathcal{M}_{x}^{t}$, à la mesure d'équidistribution $\mathcal{E}^{t}$.

Fixons pour l'instant $x \in X$ et $r>0$, et notons $V(x, r)$ l'ensemble (ouvert) des couples $(a, b) \in X^{2} \cup \partial^{2} X$ tels que la géodésique $(a b)$ rencontre $B(x, r)$. Selon le théorème 4.1.1, la mesure

$$
\nu_{x, 1}^{t}=\delta\left\|m_{\Gamma}\right\| e^{-\delta t} \sum_{(x, \gamma x) \leqslant t} \mathcal{D}_{\gamma^{-1} x} \otimes \mathcal{D}_{\gamma x}
$$

converge vers $\mu_{x} \otimes \mu_{x}$ faiblement dans $C(\bar{X})^{*}$ quand $t \rightarrow+\infty$. Restreignons désormais ces mesures à l'ouvert $V(x, r)$ (c'est-à-dire qu'on les considérera dans $\left.C_{c}(V(x, r))^{*}\right)$. Comme $1 \geqslant d_{x}(\xi, \eta)>e^{-r}$ pour $(\xi, \eta) \in \partial^{2} X \cap V(x, r)$, nous avons, pour tout $\psi \in C_{c}(V(x, r))$,

quand $t \rightarrow+\infty$.

$$
e^{-2 \delta r} \int \psi d \nu \leqslant \liminf \int \psi d \nu_{x, 1}^{t} \leqslant \limsup \int \psi d \nu_{x, 1}^{t} \leqslant \int \psi d \nu
$$

Appelons maintenant $\Gamma_{h}$ l'ensemble des isométries hyperboliques de $\Gamma$. D'après le lemme, si $\gamma \in \Gamma$ est elliptique ou parabolique, autrement dit si $\gamma \notin \Gamma_{h}$, alors $\left(\gamma^{-1} x, \gamma x\right) \notin V(x, r)$ dès que $(x, \gamma x)$ est assez grand, c'est-à-dire à l'exception d'un nombre fini de $\gamma$. Par conséquent, en notant

$$
\nu_{x, 2}^{t}=\delta\left\|m_{\Gamma}\right\| e^{-\delta t} \sum_{\substack{\gamma \in \Gamma_{h} \\(x, \gamma x) \leqslant t}} \mathcal{D}_{\gamma^{-1} x} \otimes \mathcal{D}_{\gamma x},
$$

on a $\nu_{x, 1}^{t}-\nu_{x, 2}^{t} \rightarrow 0$ quand $t \rightarrow+\infty$, toujours en restriction à $V(x, r)$.

Pour $\gamma \in \Gamma_{h}$, dont on notera $\gamma^{-}$et $\gamma^{+}$les points fixes respectivement répulsif et attractif, le lemme nous enseigne que si $\left(\gamma^{-1} x, \gamma x\right) \in V(x, r)$, alors $\gamma^{ \pm} x$ sont uniformément arbitrairement proches de $\gamma^{ \pm}$pourvu que $(x, \gamma x)$ soit assez grand, ce qui ne met à l'écart jamais qu'un nombre fini de $\gamma$. En définissant

$$
\nu_{x, 3}^{t}=\delta\left\|m_{\Gamma}\right\| e^{-\delta t} \sum_{\substack{\gamma \in \Gamma_{h} \\(x, \gamma x) \leqslant t}} \mathcal{D}_{\gamma^{-}} \otimes \mathcal{D}_{\gamma^{+}},
$$

cela entraîne que $\nu_{x, 3}^{t}-\nu_{x, 2}^{t} \rightarrow 0$ vaguement quand $t \rightarrow+\infty$, en restriction à $V(x, r)$. 
Il ressort de tout cela que

$$
e^{-2 \delta r} \int \psi d \nu \leqslant \liminf \int \psi d \nu_{x, 3}^{t} \leqslant \limsup \int \psi d \nu_{x, 3}^{t} \leqslant \int \psi d \nu
$$

pour $\psi \in C_{c}^{+}(V(x, r))$, puis pour $\psi \in C_{c}^{+}\left(\partial^{2} X \cap V(x, r)\right)$ car ces mesures sont portées par $\partial^{2} X$. Signalons que $\left.\partial^{2} X \cap V(x, r)\right)$ peut être vide sans que cela en soit pour autant préjudiciable à ce qui suit.

Pour $\gamma \in \Gamma_{h}$, notons $g_{\gamma} \subset S X$ l'axe orienté de $\gamma$, puis $\mathcal{L}_{\gamma}$ la mesure de Lebesgue le long de $g_{\gamma}$, et enfin

$$
\mathcal{M}_{x, 3}^{t}=\delta e^{-\delta t} \sum_{\substack{\gamma \in \Gamma_{h} \\(x, \gamma x) \leqslant t}} \mathcal{L}_{\gamma} .
$$

Autrement dit, $\mathcal{M}_{x, 3}^{t}=\left\|m_{\Gamma}\right\|^{-1} \nu_{x, 3}^{t} \otimes d s$. Appelons $\widehat{V}(x, r)=V(x, r) \times \mathbb{R} \subset S X$. De ce qui précède, il vient, pour $h \in C_{c}^{+}(\widehat{V}(x, r))$,

$$
e^{-2 \delta r}\left\|m_{\Gamma}\right\|^{-1} \int \varphi d m \leqslant \liminf \int \varphi d \mathcal{M}_{x, 3}^{t} \leqslant \limsup \int \varphi d \mathcal{M}_{x, 3}^{t} \leqslant\left\|m_{\Gamma}\right\|^{-1} \int \varphi d m .
$$

Notons $\ell(\gamma)$ la longueur de translation de $\gamma \in \Gamma_{h}$, et

$$
\mathcal{M}_{x}^{t}=\delta e^{-\delta t} \sum_{\substack{\gamma \in \Gamma_{h} \\ \ell(\gamma) \leqslant t}} \mathcal{L}_{\gamma} .
$$

En observant le fait élémentaire que

$$
\ell(\gamma) \leqslant(x, \gamma x) \leqslant \ell(\gamma)+2\left(x, g_{\gamma}\right)
$$

on en déduit que

$$
\mathcal{M}_{x, 3}^{t} \leqslant \mathcal{M}_{x}^{t} \leqslant e^{2 \delta r} \mathcal{M}_{x, 3}^{t+2 r}
$$

en restriction à $\widehat{V}(x, r)$.

Or, en notant $\Gamma_{h p}$ l'ensemble des isométries hyperboliques primitives de $\Gamma$, on a

$$
\mathcal{M}_{x}^{t}=\delta e^{-\delta t} \sum_{\substack{\gamma \in \Gamma_{h p} \\ \ell(\gamma) \leqslant t}}\left[\frac{t}{\ell(\gamma)}\right] \mathcal{L}_{\gamma},
$$

car

$$
\left[\frac{t}{\ell(\gamma)}\right]=\#\left\{n \in \mathbb{N}^{*} \mid \ell\left(\gamma^{n}\right)=n \ell(\gamma) \leqslant t\right\}
$$

(les crochets désignent la partie entière). Il est alors évident que

$$
\mathcal{M}_{x}^{t} \leqslant \mathcal{E}^{t}=\delta t e^{-\delta t} \sum_{\substack{\gamma \in \Gamma_{h p} \\ \ell(\gamma) \leqslant t}} \frac{1}{\ell(\gamma)} \mathcal{L}_{\gamma} .
$$


Pour obtenir une inégalité complémentaire, considérons $h \in C_{c}^{+}(\widehat{V}(x, r))$, et observons d'abord que $[t / \ell(\gamma)] \geqslant 1 / \ell(\gamma)$ si $\ell(\gamma) \leqslant t$ et $t \geqslant 2$, d'où

$$
\sum_{\substack{\gamma \in \Gamma_{h p} \\ \ell(\gamma) \leqslant t}} \frac{1}{\ell(\gamma)} \int \varphi d \mathcal{L}_{\gamma}=O\left(e^{\delta t}\right),
$$

comme l'on a vu que $\int \varphi d \mathcal{M}_{x}^{t}$ est borné, pour $t \geqslant 2$. Ensuite, si $e^{-r} t<\ell(\gamma) \leqslant t$, alors $[t / \ell(\gamma)] \geqslant 1 \geqslant e^{-r} t / \ell(\gamma)$ d'où

$$
\begin{aligned}
\int h d \mathcal{M}_{x}^{t} & \geqslant e^{-r} \delta t e^{-\delta t} \sum_{\substack{\gamma \in \Gamma_{h p} \\
e^{-r} t<\ell(\gamma) \leqslant t}} \frac{1}{\ell(\gamma)} \int \varphi d \mathcal{L}_{\gamma} \\
& =e^{-r} \int \varphi d \mathcal{E}^{t}-e^{-r} \delta t e^{-\delta t} \sum_{\substack{\gamma \in \Gamma_{h p} \\
\ell(\gamma) \leqslant e^{-r} t}} \frac{1}{\ell(\gamma)} \int \varphi d \mathcal{L}_{\gamma} .
\end{aligned}
$$

Mais par ce qui précède, le second terme de la différence ci-dessus est majoré par une constante fois $t e^{\delta\left(e^{-r}-1\right) t}=o(1)$. Aussi obtient-on que

$$
\limsup \int h d \mathcal{M}_{x}^{t} \geqslant e^{-r} \limsup \int \varphi d \mathcal{E}^{t} \text {. }
$$

En fin de compte (en remontant à $\mathcal{M}_{x, 3}^{t}$ ), nous avons établi que pour toute $h \in C_{c}^{+}(\widehat{V}(x, r))$, lorsque $t \rightarrow+\infty$,

$e^{-2 \delta r}\left\|m_{\Gamma}\right\|^{-1} \int \varphi d m \leqslant \liminf \int \varphi d \mathcal{E}^{t} \leqslant \lim \sup \int \varphi d \mathcal{E}^{t} \leqslant e^{(2 \delta+1) r}\left\|m_{\Gamma}\right\|^{-1} \int \varphi d m$.

En recourant à une partition de l'unité localement finie et subordonnée au recouvrement de $S X$ par les $\widehat{V}(x, r), x \in X$, avec $r>0$ fixé, on étend la validité de l'encadrement précédent à n'importe quelle fonction $h \in C_{c}^{+}(S X)$. Il ne reste plus qu'à faire tendre $r$ vers zéro pour achever la preuve.

Si $\Gamma$ est convexe-cocompact, il découle directement du théorème 5.1.1 que

$$
\# \mathcal{G}_{\Gamma}(\ell) \sim \frac{e^{\delta \ell}}{\delta \ell} \quad \text { quand } \ell \longrightarrow \infty
$$

et l'on retrouve un résultat déjà connu (voir entre autres [Hub] pour les surfaces compactes, $[\mathbf{M a r g}]$ pour le cas des variétés compactes, $[\mathbf{L}]$ pour les convexe-cocompactes). Cependant, la même estimation a pu être étendue pour certaines variétés géométriquement finies avec des pointes (voir [Dal-P] et leur références). L'objet du théorème suivant est de généraliser ce résultat pour tous les groupes géométriquement finis (avec la définition étendue que nous en donnons pour les espaces CAT $(-1)$ au paragraphe $1 \mathrm{~F}$ ) et admettant en outre une mesure de BMS finie (ce qui, en courbure variable, ne découle plus de la première hypothèse, comme cela a été rappelé en $1 \mathrm{~F}$, d'après [D-O-P]). La contrainte géométrique nous a paru nécessaire afin de cerner le comportement des mesures d'équidistributions du théorème 5.1.1 (on est ici ramené à les 
estimer au niveau des pointes paraboliques) ; en fait, il est même vraisemblable qu'il puisse exister des groupes à mesure de BMS finie qui ne soient pas géodésiquement finis, c'est-à-dire tels que $\# \mathcal{G}_{\Gamma}(\ell)$ ne soit pas fini pour tout $\ell$, tandis que ce fait est bien connu pour les groupes géométriquement finis.

Nous désignons ci-dessous par $C_{b}(S X / \Gamma)^{*}$ le dual faible-* de l'espace des fonctions continues bornées sur $S X / \Gamma$.

THÉORÈme 5.2. - Supposons que $\Gamma$ soit géométriquement fini et admette une mesure de Bowen-Margulis-Sullivan $m_{\Gamma}$ finie. Alors, quand $\ell \rightarrow+\infty$,

$$
\delta \ell e^{-\delta \ell} \sum_{g \in \mathcal{G}_{\Gamma}(\ell)} \mathcal{D}_{g} \rightarrow \frac{m_{\Gamma}}{\left\|m_{\Gamma}\right\|} \text { faiblement dans } C_{b}(S X / \Gamma)^{*} .
$$

Corollaire 5.3. - $\quad \# \mathcal{G}_{\Gamma}(\ell) \sim \frac{e^{\delta \ell}}{\delta \ell}$ quand $\ell \rightarrow+\infty$.

Démonstration. - Notons $\mathcal{E}_{\Gamma}^{\ell}$ la mesure $\delta \ell e^{-\delta \ell} \sum_{g \in \mathcal{G}_{\Gamma}(\ell)} \mathcal{D}_{g}$ sur $S X / \Gamma$. Par le théorème 5.1.1, nous savons déjà que $\mathcal{E}_{\Gamma}^{\ell} \rightarrow m_{\Gamma} /\left\|m_{\Gamma}\right\|$ faiblement dans $C_{c}(S X / \Gamma)^{*}$ quand $\ell \rightarrow+\infty$. Commençons par substituer à $\mathcal{E}_{\Gamma}^{\ell}$ une mesure voisine mais qui sera mieux adaptée à ce qui va suivre, en définissant

$$
\mathcal{M}_{\Gamma}^{\ell}=\delta e^{-\delta \ell} \sum_{g \in \mathcal{G}_{\Gamma}(\ell)} \ell(g) \mathcal{D}_{g}
$$

(remarquons que $\ell(g) \mathcal{D}_{g}$ est simplement la mesure de Lebesgue le long de $g$ non normalisée). Le premier point à vérifier est que l'on a encore $\mathcal{M}_{\Gamma}^{\ell} \rightarrow m_{\Gamma} /\left\|m_{\Gamma}\right\|$ faiblement dans $C_{c}(S X / \Gamma)^{*}$ quand $\ell \rightarrow+\infty$. En effet, si $\varphi \in C_{c}(S X / \Gamma), \varphi \geqslant 0$, on a d'une part

$$
\int \varphi d \mathcal{M}_{\Gamma}^{\ell} \leqslant \int \varphi d \mathcal{E}_{\Gamma}^{\ell}
$$

et d'autre part, avec $\varepsilon>0$ arbitrairement petit,

$$
\begin{aligned}
\int \varphi d \mathcal{M}_{\Gamma}^{\ell} & \geqslant \delta e^{-\delta \ell} \int \varphi \sum_{e^{-\varepsilon} \ell<\ell(g) \leqslant \ell} \ell(g) d \mathcal{D}_{g} \geqslant e^{-\varepsilon} \delta \ell e^{-\delta \ell} \int \varphi \sum_{e^{-\varepsilon} \ell<\ell(g) \leqslant \ell} d \mathcal{D}_{g} \\
& \geqslant e^{-\varepsilon} \int \varphi d \mathcal{E}_{\Gamma}^{\ell}-e^{-\left(1-e^{-\varepsilon}\right) \delta \ell} \int \varphi d \mathcal{E}_{\Gamma}^{e^{-\varepsilon} \ell} .
\end{aligned}
$$

On conclut de là que

quand $\ell \rightarrow+\infty$, comme annoncé.

$$
\int \varphi d \mathcal{M}_{\Gamma}^{\ell} \longrightarrow \int \varphi \frac{d m_{\Gamma}}{\left\|m_{\Gamma}\right\|}
$$

Nous allons prouver ci-dessous que $\mathcal{M}_{\Gamma}^{\ell}$ converge faiblement dans $C_{b}(S X / \Gamma)^{*}$ vers $m_{\Gamma} /\left\|m_{\Gamma}\right\|$ quand $\ell \rightarrow+\infty$. La même conclusion vaudra alors également pour $\mathcal{E}_{\Gamma}^{\ell}$, puisque pour $\varphi \in C_{b}(S X / \Gamma)$ positive, on aura d'une part

$$
\int \varphi d \mathcal{E}_{\Gamma}^{\ell} \geqslant \int \varphi d \mathcal{M}_{\Gamma}^{\ell}
$$


et d'autre part, avec $\varepsilon>0$ arbitrairement petit, en notant $\ell_{0}$ la plus petite longueur de géodésique fermée sur $S X / \Gamma$ (qui est géodésiquement fini),

$$
\begin{aligned}
\int \varphi d \mathcal{E}_{\Gamma}^{\ell} & =\delta \ell e^{-\delta \ell} \int \varphi \sum_{\ell(g) \leqslant e^{-\varepsilon} \ell} d \mathcal{D}_{g}+\delta \ell e^{-\delta \ell} \int \varphi \sum_{e^{-\varepsilon} \ell<\ell(g) \leqslant \ell} d \mathcal{D}_{g} \\
& \leqslant \frac{1}{\ell_{0}} \delta \ell e^{-\delta \ell} \int \varphi \sum_{\ell(g) \leqslant e^{-\varepsilon} \ell} \ell(g) d \mathcal{D}_{g}+e^{\varepsilon} \delta e^{-\delta \ell} \int \varphi \sum_{e^{-\varepsilon} \ell<\ell(g) \leqslant \ell} \ell(g) d \mathcal{D}_{g} \\
& \leqslant \frac{1}{\ell_{0}} \ell e^{-\left(1-e^{-\varepsilon}\right) \delta \ell} \int \varphi d \mathcal{M}_{\Gamma}^{e^{-\varepsilon} \ell}+e^{\varepsilon} \int \varphi d \mathcal{M}_{\Gamma}^{\ell}=e^{\varepsilon} \int \varphi \frac{d m_{\Gamma}}{\left\|m_{\Gamma}\right\|}+o(1) .
\end{aligned}
$$

On en conclura que

quand $\ell \rightarrow+\infty$, ce qu'il fallait montrer.

$$
\int \varphi d \mathcal{E}_{\Gamma}^{\ell} \longrightarrow \int \varphi \frac{d m_{\Gamma}}{\left\|m_{\Gamma}\right\|}
$$

Décrivons pour commencer le soubassement géométrique de la situation présente. Choisissons un domaine $D$ fondamental pour l'action de $\Gamma$ sur $X$, localement fini, de bord négligeable pour la mesure $S^{*} m$ donnée par $S^{*} m(B)=m(S B)$ (voir 1C, vu que l'ensemble des points fixes de $\Gamma$ dans $X$, étant constitué du projeté de ceux dans $S X$ et de points isolés, reste négligeable pour $S^{*} m$ ). Aussi $S D$ est-il un domaine fondamental pour l'action de $\Gamma$ sur $S X$ localement fini et de bord $m$-négligeable. Prenons un système $\mathcal{P}$ de représentants des orbites suivant $\Gamma$ des points fixes paraboliques de $\Gamma$ et, pour $p \in \mathcal{P}$, appelons $\Pi_{p}$ le sous-groupe (parabolique maximal) de $\Gamma$ stabilisant $p$. Par la finitude géométrique de $\Gamma$, on sait que $\mathcal{P}$ est fini et que pour chaque $p \in \mathcal{P}$ on peut trouver une horoboule $H_{p}$ basée en $p$ telle que pour tout $\gamma \in \Gamma, \gamma H_{p}$ rencontre $H_{p}$ si et seulement si $\gamma \in \Pi_{p}$ (voir le paragraphe $1 \mathrm{~F}$ ). Pour $r \geqslant 0$, notons $H_{p}(r)$ l'horoboule contenue dans $H_{p}$ et dont le bord est à distance $r$ de celui de $H_{p}$, puis

$$
D_{r}=D \backslash \bigcup_{p \in \mathcal{P}} \Gamma H_{p}(r)
$$

et appelons $\mathcal{C}$ la réunion des géodésiques dans $X$ à extrémités dans $\Lambda(\Gamma)$; on sait également que l'intersection de $D_{r}$ et de $\mathcal{C}$ est compacte dans $X$.

Appelons $\mathcal{M}^{\ell}$ la mesure sur $S X$ au-dessus de $\mathcal{M}_{\Gamma}^{\ell}$. Pour alléger les notations dans ce qui suit, il sera commode de désigner par $S^{*} \mathcal{M}^{\ell}$ la mesure sur $X$ définie par $S^{*} \mathcal{M}^{\ell}(B)=\mathcal{M}^{\ell}(S B)$ pour tout borélien $B$ de $X$. Compte tenu du fait que $\mathcal{M}_{\Gamma}^{\ell}$ converge faiblement dans $C_{c}(S X / \Gamma)^{*}$ vers $m_{\Gamma} /\left\|m_{\Gamma}\right\|$ quand $\ell \rightarrow+\infty$, et que l'intersection de $S D_{r}$ et du support de $m$, lequel contient en outre ceux des mesures $\mathcal{M}^{\ell}$, est compacte pour chaque $r \geqslant 0$, il suffit de montrer que

$$
\limsup _{\ell \rightarrow+\infty} S^{*} \mathcal{M}^{\ell}\left(D \backslash D_{r}\right) \longrightarrow 0 \text { quand } r \longrightarrow+\infty,
$$

ou encore que pour chaque $p \in \mathcal{P}$, on a

$$
\limsup _{\ell \rightarrow+\infty} S^{*} \mathcal{M}^{\ell}\left(D \cap \Gamma H_{p}(r)\right) \longrightarrow 0 \quad \text { quand } r \longrightarrow+\infty \text {. }
$$


Fixons dorénavant $p \in \mathcal{P}$. Nous omettrons dans tout ce qui suit l'indice $p$ afin d'alléger les notations précédemment introduites. Choisissons un domaine $F$ relativement compact et fondamental stricto sensu pour l'action de $\Pi \operatorname{sur} \Lambda(\Gamma) \backslash\{p\}$. Quitte à modifier le domaine fondamental $D$ localement fini pour $\Gamma$ que nous avions choisi, il nous est loisible de supposer que $D \cap \Gamma H=D \cap H$, et par suite que $D \cap \Gamma H(r)=D \cap H(r)$ pour $r \geqslant 0$.

Pour une isométrie hyperbolique $\gamma \in \Gamma$, notons $g_{\gamma} \subset X$ son axe non orienté, puis $\mathcal{L}_{\gamma}$ la mesure de Lebesgue le long de $g_{\gamma}$. Nous avons $S^{*} \mathcal{M}^{\ell}=\delta e^{-\delta \ell} \sum \mathcal{L}_{\gamma}$ où la somme porte sur les isométries $\gamma$ hyperboliques primitives de longueur de translation au plus $\ell$. Comme les extrémités de $g_{\gamma}$ sont dans $\Lambda(\Gamma) \backslash\{p\}=\bigcup_{\pi \in \Pi} \pi F$, on peut écrire que

$$
S^{*} \mathcal{M}^{\ell}(D \cap H(r))=\delta e^{-\delta \ell} \sum_{\pi_{1} \in \Pi} \sum_{\pi_{2} \in \Pi} \sum \mathcal{L}_{\gamma}(D \cap H(r))
$$

où la troisième somme est prise sur les isométries $\gamma$ hyperboliques primitives de longueur de translation au plus $\ell$ et dont l'axe va de $\pi_{1} F$ à $\pi_{2} F$. En écrivant que $\mathcal{L}_{\gamma}(D \cap H(r))=\mathcal{L}_{\pi_{1}^{-1} \gamma \pi_{1}}\left(\pi_{1}^{-1}(D \cap H(r))\right)$, puis en réindexant la seconde somme avec $\pi=\pi_{1}^{-1} \pi_{2}$, et enfin la troisième en y remplaçant $\pi_{1}^{-1} \gamma \pi_{1}$ par $\gamma$, il vient que

$$
S^{*} \mathcal{M}^{\ell}(D \cap H(r))=\delta e^{-\delta \ell} \sum_{\pi_{1} \in \Pi} \sum_{\pi \in \Pi} \sum_{\gamma \in \Gamma(\ell, \pi)} \mathcal{L}_{\gamma}\left(\pi_{1}^{-1}(D \cap H(r))\right)
$$

où $\Gamma(\ell, \pi)$ est l'ensemble des isométries hyperboliques primitives dans $\Gamma$ de longueur de translation au plus $\ell$ et dont l'axe va de $F$ à $\pi F$. Or $\bigcup_{\pi_{1} \in \Pi} \pi_{1}^{-1}(D \cap H(r))=H(r)$, d'où finalement

$$
S^{*} \mathcal{M}^{\ell}(D \cap H(r))=\delta e^{-\delta \ell} \sum_{\pi \in \Pi} \sum_{\gamma \in \Gamma(\ell, \pi)} \mathcal{L}_{\gamma}(H(r))
$$

Nous nous proposons de majorer la quantité $\sum_{\gamma \in \Gamma(\ell, \pi)} \mathcal{L}_{\gamma}(H(r))$. Choisissons une géodésique allant de $F$ à $p$, et désignons par $x$ le point commun à cette géodésique et à l'horosphère $\partial H$. Nous utiliserons le lemme suivant, que l'on obtient par comparaison avec le demi-plan hyperbolique, et dont nous laisserons au lecteur le soin de vérifier les détails. Rappelons auparavant que les horoboules sont strictement convexes.

LEMME. - Si une géodésique g partant de F pénètre l'horoboule fermée $\bar{H}(r \geqslant 0)$ au point $y(y \in \partial H)$, alors $(x, y) \leqslant \frac{1}{2} \kappa$, où $\kappa$ est une constante ne dépendant pas de $g$.

Indiquons succinctement le moyen d'obtenir ce lemme. On substitue d'abord à $F$ un point de lui-même ; c'est à ce moment-là que l'on use d'une comparaison avec le demi-plan hyperbolique, et l'énoncé est valable avec une constante absolue à la place de $\kappa$. On en déduit aisément l'énoncé final grâce à la relative compacité de $F$ dans $\partial X \backslash\{p\}$. 
Considérons maintenant $\gamma \in \Gamma(\ell, \pi)$ tel que $\mathcal{L}_{\gamma}(H(r))>0$. L'axe $g_{\gamma}$ rencontre donc $H(r)$. Mettons que $g_{\gamma}$ pénètre $\bar{H}$ au point $a$, puis $\overline{H(r)}$ au point $b$, ensuite ressort de $\overline{H(r)}$ au point $c$, enfin de $\bar{H}$ au point $d$. Par le lemme ci-dessus, nous avons d'une part $(a, x) \leqslant \frac{1}{2} \kappa$, d'autre part $(d, \pi x)=\left(\pi^{-1} d, x\right) \leqslant \frac{1}{2} \kappa$ puisque l'axe $\pi^{-1} g_{\gamma}$ parcouru en sens inverse part de $F$ et pénètre $\pi^{-1} \bar{H}=\bar{H}$ au point $\pi^{-1} d$. De plus, $(a, b) \geqslant r$ et $(c, d) \geqslant r$. Il apparaît ainsi que

$$
\mathcal{L}_{\gamma}(H(r))=(b, c)=(a, d)-(a, b)-(c, d) \leqslant(x, \pi x)+\kappa-2 r .
$$

On a notamment que $(x, \pi x) \geqslant 2 r-\kappa$.

Remarquons aussi que $\gamma a$ étant situé sur le rayon géodésique $] a, d) \subset g_{\gamma}$, et l'intervalle $] a, d[$ étant contenu dans $H$, l'on a nécessairement $(a, \gamma a) \geqslant(a, d) \geqslant 2 r$, puis $(x, \gamma x) \geqslant 2 r-\kappa$. En outre, $(x, \gamma x) \leqslant(a, \gamma a)+\kappa=\ell(\gamma)+\kappa \leqslant \ell+\kappa$. Nous aurons besoin de ces faits un peu plus loin.

Après avoir majoré $\mathcal{L}_{\gamma}(H(r))$ ci-dessus, nous allons pour finir majorer le cardinal de l'ensemble $\Gamma(\ell, \pi, r)$ des $\gamma \in \Gamma(\ell, \pi)$ tels que $\mathcal{L}_{\gamma}(H(r))>0$, en recourant au lemme de l'ombre (lemme 1.3) appliqué à la densité $\mu$ conforme de dimension $\delta$ et invariante par $\Gamma$ associée à $m$. Prenons $R>\kappa$ assez grand pour que l'énoncé de ce lemme soit valable pour les ombres des boules $B(\gamma x, R)$ vues de $x$. Comme l'axe $g_{\gamma}$ est à distance au plus $\frac{1}{2} \kappa<\frac{1}{2} R$ de $x$ ainsi que de $\gamma x$, l'ombre $\mathcal{O}_{\gamma}$ de $B(\gamma x, R)$ vue de $x$ contient le point attractif de $\gamma$, et partant rencontre $\pi F$. Dès que $\left(x, \pi^{-1} x\right)$ est assez grand, ce dont on s'assure, d'après ce qui précède, en prenant $r$ lui-même assez grand dans tout ce qui suit, le point $\pi^{-1} x$ est proche de $p$, et comme $\pi^{-1} \mathcal{O}_{\gamma}$ est l'ombre de $B\left(\pi^{-1} \gamma x, R\right)$ vue de $\pi^{-1} x$ et qu'elle rencontre $F$, lequel est relativement compact dans $\Lambda(\Gamma) \backslash\{p\}$, on voit que $\pi^{-1} \mathcal{O}_{\gamma}$ est contenue dans un compact $K \subset \partial X \backslash\{p\}$ indépendant des variables en jeu. Ainsi, pour $\gamma \in \Gamma(\ell, \pi, r)$, on a $\mathcal{O}_{\gamma} \subset \pi^{-1} K$. Or pour tout $t>0$, la famille des $\mathcal{O}_{\gamma}$ avec $\gamma \in \Gamma(\ell, \pi, r)$ tel que $t-1<(x, \gamma x) \leqslant t$ forme un recouvrement de multiplicité bornée uniformément, et comme $\mu_{x}\left(\mathcal{O}_{\gamma}\right)$ est plus grand qu'une constante fois $e^{-\delta t}$ d'après le lemme 1.3, il en ressort que ces $\gamma$ sont en nombre inférieur à une constante fois $e^{\delta t} \mu_{x}\left(\pi^{-1} K\right.$ ) (c'est un avatar de l'argument bien connu évoqué au paragraphe $1 \mathrm{~B})$. Pour $\gamma \in \Gamma(\ell, \pi, r)$, on a vu plus haut que $(x, \gamma x) \leqslant \ell+\kappa$. Aussi, par sommation de l'estimée précédente par rapport à $t$, en déduit-on que le cardinal de $\Gamma(\ell, \pi, r)$ est majoré par une constante fois $e^{\delta \ell} \mu_{x}\left(\pi^{-1} K\right)$.

Reste à majorer $\mu_{x}\left(\pi^{-1} K\right)$. On a

$$
\mu_{x}\left(\pi^{-1} K\right)=\mu_{\pi x}(K)=\int_{K} e^{-\delta \beta_{\xi}(\pi x, x)} d \mu_{x}(\xi) .
$$

Or, dès que $r$, et donc $(x, \pi x)$, est assez grand, le point $\pi x$ est proche de $p$, et comme $K$ est compact dans $\partial X \backslash\{p\}$, on voit que $K$ est contenu dans l'ombre d'une boule de centre $x$, de rayon fixé indépendamment de $\pi$, vue de $\pi x$. Une application du lemme 1.2 nous montre alors que $\mu_{x}\left(\pi^{-1} K\right)$ est majoré par une constante fois $e^{-\delta(x, \pi x)}$. Avec 
ce qui a été vu plus haut, on obtient en fin de compte que le cardinal de $\Gamma(\ell, \pi, r)$ est plus petit qu'une constante fois $e^{\delta \ell-\delta(x, \pi x)}$.

En rassemblant ce qui précède, on obtient finalement la majoration

$$
S^{*} \mathcal{M}^{\ell}(D \cap H(r)) \leqslant C \sum_{\substack{\pi \in \Pi \\(x, \pi x)>2 r-\kappa}}((x, \pi x)-2 r+\kappa) e^{-\delta(x, \pi x)}
$$

où $C$ est une constante indépendante de $\ell$ et de $r$.

Les hypothèses auxquelles satisfait $\Gamma$ font que la série $\sum_{\pi \in \Pi(x, \pi x) e^{-\delta(x, \pi x)}}$ converge ( $c f$. la proposition issue de [D-O-P]). Par conséquent,

$$
\limsup _{\ell \rightarrow+\infty} S^{*} \mathcal{M}^{\ell}(D \cap H(r)) \longrightarrow 0 \text { quand } r \longrightarrow+\infty,
$$

ce qu'il fallait montrer.

REMARQue. - On peut voir en suivant pas à pas cette preuve que le reste de série obtenu ci-dessus en majoration de $S^{*} \mathcal{M}^{\ell}(D \cap H(r))$ est en fait proportionnel (avec des constantes larges) à cette quantité.

Lorsque $X$ est l'espace hyperbolique de dimension $d+1$ et de courbure -1 , on sait que $\Pi$ est une extension finie d'un groupe isomorphe à $\mathbb{Z}^{k}$ (l'entier $k$ est appelé le rang de $\Pi$ ). On sait de plus que $k<2 \delta$. Il s'avère dans ces conditions que le reste de série ci-dessus

$$
\sum_{(x, \pi x)>2 r-\kappa}((x, \pi x)-2 r+\kappa) e^{-\delta(x, \pi x)}
$$

est proportionnel à $e^{(k-2 \delta) r}$. On retrouve ainsi des estimées connues (voir [Sull3] par exemple). 


\section{CHAPITRE 6}

\section{MOYENNES HOROSPHÉRIQUES ET CLASSIFICATION DES MESURES INVARIANTES}

$\mathrm{Au}$ terme de ce travail, nous revenons sur les questions d'ergodicité du feuilletage horosphérique, qui nous avaient déjà occupés dans le chapitre 2. Tous les groupes considérés ici seront supposés admettre une mesure de BMS finie, et toujours un spectre des longueurs non arithmétique (cela depuis le chapitre 3 ).

Nous allons d'abord approfondir le corollaire 2.3 en établissant un théorème ergodique relatif à la convergence de moyennes sur les horosphères (in)stables du flot géodésique (théorème 6.1). Comme il s'avérera que ces moyennes convergent — dans une certaine mesure - sur toute horosphère basée en un point limite conique, nous serons alors conduits à un résultat d'unique ergodicité du feuilletage horosphérique au niveau des points limites coniques (théorème 6.4); comme application, nous déterminons, pour un groupe supposé en outre géométriquement fini, toutes les mesures de Radon invariantes par le feuilletage horosphérique (c'est-à-dire toutes les mesures de Radon sur $\mathcal{H}=\partial X \times \mathbb{R}$ invariantes par $\Gamma$ ). Ces résultats reposent entièrement sur le mélange du flot géodésique affirmé par le théorème 3.1.

Le théorème suivant peut être considéré comme une extension faible du théorème ergodique de Birkhoff. Dans le cas particulier où $X$ est le disque hyperbolique et où $X / \Gamma$ est d'aire finie $\left(m_{\Gamma}\right.$ est alors la mesure de Liouville, $\mu$ la densité de Lebesgue et $\delta(\Gamma)$ est maximal égal à 1 ), ce dernier théorème énonce que la convergence ci-dessous a lieu en fait presque partout, puisque la moyenne sur $B^{+}(u, r)$ est celle classiquement associée au flot horocyclique instable $h^{t}$, à savoir

$$
\frac{1}{2 r} \int_{-r}^{r} \widetilde{\varphi} \circ h^{t}(u) d t,
$$

et que la mesure de Liouville est invariante par $h^{t}$. Mais que $\delta(\Gamma)$ soit non maximal ou que l'on se place en dimension supérieure, il s'en faut d'un tel théorème ergodique abstrait; bien pis, dans la situation générale envisagée ici, l'on ne peut que constater l'absence d'une action de groupe (pour un flot, il s'agit de $\mathbb{R}$ ) déterminant les 
horosphères de l'espace CAT $(-1) X$. Quoi qu'il en soit, il faut surtout noter que les moyennes horosphériques ici proposées en remplacement ne rappellent pas tant les moyennes de Hopf que celles de Birkhoff, en dépit de la variabilité spatiale du dénominateur, et qu'on les étudie sous la mesure finie $m_{\Gamma}$, tandis qu'en dimension deux, cette dernière n'est généralement rien moins qu'invariante par le flot horocyclique, qui $\mathrm{du}$ reste n'admet pas toujours de mesure invariante finie (ce qui ressort notamment du corollaire 6.5 plus loin). C'est encore là une raison pour laquelle il faut, jusqu'en dimension deux, préférer au flot horocyclique le feuilletage du même nom : c'est que la paramétrisation convenable des horocycles n'est généralement pas celle de Lebesgue, mais répond à la densité de Patterson-Sullivan.

La démonstration de ce théorème 6.1 confirme le fait avancé plus haut (chapitre 3 ) que mélange du flot géodésique et ergodicité du feuilletage horosphérique - une conséquence immédiate de l'énoncé ci-dessous - étaient bien deux problèmes équivalents lorsque $\Gamma$ admet une mesure de BMS finie. Rappelons que nous avions adopté la démarche réciproque pour aboutir au théorème 3.1 à partir du corollaire 2.3.

THÉORÈme 6.1. - Supposons que $\Gamma$ admette une mesure de Bowen-Margulis-Sullivan $m_{\Gamma}$ finie, associée à une densité $\mu$. Soit $\varphi$ une fonction dans $L^{1}\left(m_{\Gamma}\right)$, que l'on relève en une fonction $\widetilde{\varphi}$ sur $S X$. Pour $r>0$, soit $M_{r}(\varphi)$ l'application mesurable

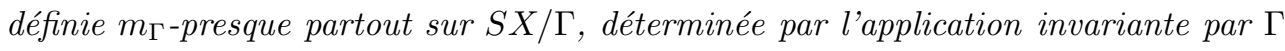
qui à m-presque tout $u \in S X$ associe

$$
\frac{1}{\mu_{H^{+}(u)}\left(B^{+}(u, r)\right)} \int_{B^{+}(u, r)} \tilde{\varphi} d \mu_{H^{+}(u)}
$$

Supposons $h$ bornée. Alors

$$
M_{r}(\varphi) \longrightarrow \frac{1}{\left\|m_{\Gamma}\right\|} \int \varphi d m_{\Gamma} \quad \text { dans } L^{1}\left(m_{\Gamma}\right) \text { quand } r \longrightarrow+\infty .
$$

À noter que $\mu_{H^{+}(u)}\left(B^{+}(u, r)\right)>0$ dès que $u$ est dans le support de $m$, aussi $M_{r}(\varphi)$ est-elle bien définie.

La convergence ci-dessus est encore valide même lorsque $h$ n'est pas bornée, pourvu que la condition $(*)$ énoncée plus loin soit remplie (voir proposition 6.3 ; la raison en est indiquée dans la troisième étape de la preuve du théorème 6.4 ).

Démonstration. - Nous allons d'abord montrer le théorème dans le cas où $\varphi$ est continue à support compact, puis nous en tirerons le cas général.

Soit $\varphi \in C_{c}(S X / \Gamma)$. Pour tout $u$ dans le support de $m_{\Gamma}$, le corollaire 3.2, appliqué avec $E^{+}=B^{+}(u, 1)$, signifie exactement que

$$
M_{1}\left(h \circ g_{\Gamma}^{t}\right)(u) \longrightarrow \frac{1}{\left\|m_{\Gamma}\right\|} \int \varphi d m_{\Gamma} \quad \text { quand } t \longrightarrow+\infty .
$$


Or en effectuant un simple changement de variable (pour lequel on pourra s'aider du paragraphe $1 \mathrm{G})$, on voit que $M_{1}\left(h \circ g_{\Gamma}^{t}\right)=M_{r}(h) \circ g_{\Gamma}^{t}$ avec $r=e^{t}$. Le résultat voulu s'ensuit, grâce à l'invariance de $m_{\Gamma}$ par le flot géodésique.

Venons-en au cas général. Sans la condition $(*)$ (voir plus loin), nous aurons en général besoin de l'ensemble $N_{\kappa}(\kappa>0)$, projeté dans $S X / \Gamma$ de

$$
\widetilde{N_{\kappa}}=\left\{v \in S X \mid \forall u \in B^{+}(v, 1), \mu_{H^{+}(u)}\left(B^{+}(u, 1)\right) \leqslant \kappa \mu_{H^{+}(v)}\left(B^{+}(v, 1)\right)\right\} .
$$

Comme $N_{\kappa}$ croît avec $\kappa$ et que $\bigcup_{\kappa>0} N_{\kappa}$ contient le support de $m_{\Gamma}$, on a

$$
m_{\Gamma}\left(N_{\kappa}\right) \longrightarrow\left\|m_{\Gamma}\right\| \text { quand } \kappa \longrightarrow \infty \text {. }
$$

Nous allons montrer ci-dessous que pour toute $\psi \in L^{1}\left(m_{\Gamma}\right)$, on a

$$
\int_{g_{\Gamma}^{\log r} N_{\kappa}} M_{r}(\psi) d m_{\Gamma} \leqslant \kappa \int|\psi| d m_{\Gamma}
$$

Grâce à la densité de $C_{c}(S X / \Gamma)$ dans $L^{1}\left(m_{\Gamma}\right)$, on déduira aisément le théorème de ce qui précède, pourvu que $h$ soit bornée.

Reste à prouver l'inégalité (maximale) précédente, ce qui requerra un lemme dont le principe nous sera également fort utile dans les deux démonstrations suivantes, ce qui lui a valu d'être rejeté à la fin de la présente preuve. Une application de ce lemme nous donne en effet l'identité suivante, où l'on pose $t=\log r$ :

$$
\begin{aligned}
\int d m_{\Gamma}(u) \mathbb{1}_{N_{\kappa}}\left(g_{\Gamma}^{-t} u\right) \frac{1}{\mu_{H^{+}(u)}\left(B^{+}(u, r)\right)} \int_{B^{+}(u, r)} \widetilde{\psi} d \mu_{H^{+}(u)} \\
=\int d m_{\Gamma}(u) \psi(u) \int_{B^{+}(u, r)} \frac{\mathbb{1}_{\widetilde{N}_{\kappa}}\left(g^{-t} v\right)}{\mu_{H^{+}(v)}\left(B^{+}(v, r)\right)} d \mu_{H^{+}(u)}(v) .
\end{aligned}
$$

Or le second membre est certainement plus petit que $\kappa \int|\psi| d m_{\Gamma}$ par définition de $N_{\kappa}$.

Pour finir, énonçons et justifions la propriété d'auto-adjonction suivante, dont nous nous sommes servis à l'instant. Il n'y est plus nécessaire de supposer que $m_{\Gamma}$ soit finie.

LEMME. - Soient $\varphi$ et $\psi$ deux fonctions boréliennes sur $S X / \Gamma$, que l'on relève en $\widetilde{h}$ et $\widetilde{\psi}$ respectivement sur $S X$, et $r>0$. Alors

$$
\int d m_{\Gamma}(u) \varphi(u) \int_{B^{+}(u, r)} \widetilde{\psi}(v) d \mu_{H^{+}(u)}(v)=\int d m_{\Gamma}(u) \psi(u) \int_{B^{+}(u, r)} \widetilde{\varphi}(v) d \mu_{H^{+}(u)}(v),
$$

où la notation

$$
\int_{B^{+}(u, r)} \widetilde{\psi}(v) d \mu_{H^{+}(u)}(v)
$$

désigne abusivement la quantité bien définie lorsque l'on y substitue à u n'importe quel relevé de ce point dans $S X$. 
Démonstration. - Nous nous appuierons sur la proposition 1.12. Aussi fixons $u_{0} \in$ $S X$ (avec $\left.\mu_{o}\left(g^{+\infty} u_{0}\right)=0\right)$ et notons $d \nu\left(H^{+}\right)$la mesure sur l'espace des horosphères instables, invariante par $\Gamma$, donnée par

$$
d \nu\left(H^{+}(v)\right)=\int_{\mathbb{R}} d t \int_{H^{-}\left(g^{t} u_{0}\right)} d \mu_{H^{-}\left(g^{t} u_{0}\right)}(v) .
$$

Nous supposerons pour alléger que le groupe $\Gamma$ est ici dépourvu de torsion; dans le cas général, il suffit de restreindre $m$ aux points d'ordre donné, et de reprendre ce qui suit (qui correspond à l'ordre 1), ceci pour chaque ordre. Prenons donc un domaine $D$ fondamental pour l'action de $\Gamma$ sur $S X$ localement fini et de bord $m$-négligeable (voir 1C). Le membre de gauche dans l'énoncé vaut

$$
\int d \nu\left(H^{+}\right) \int_{H^{+}} d \mu_{H^{+}}(u) \mathbb{1}_{D}(u) \widetilde{\varphi}(u) \int_{B^{+}(u, r)} d \mu_{H^{+}}(v) \widetilde{\psi}(v),
$$

ou encore, grâce au théorème de Fubini,

$$
\int d \nu\left(H^{+}\right) \int_{H^{+} \times H^{+}} d\left(\mu_{H^{+}} \otimes \mu_{H^{+}}\right)(u, v) \mathbb{1}_{D}(u) \widetilde{\varphi}(u) \widetilde{\psi}(v) \mathbb{1}_{B^{+}(u, r)}(v) .
$$

En effectuant une manipulation élémentaire avec $\Gamma$ et $D$, et en observant que $\mathbb{1}_{B^{+}(u, r)}(v)=\mathbb{1}_{B^{+}(v, r)}(u)$, la quantité précédente vaut également :

$$
\int d \nu\left(H^{+}\right) \int_{H^{+} \times H^{+}} d\left(\mu_{H^{+}} \otimes \mu_{H^{+}}\right)(u, v) \widetilde{\varphi}(u) \mathbb{1}_{D}(v) \widetilde{\psi}(v) \mathbb{1}_{B^{+}(v, r)}(u) .
$$

Cette expression est symétrique à la précédente en $h$ et $\psi$, et la conclusion s'ensuit.

Le restant de ce chapitre est consacré au problème d'unique ergodicité du feuilletage horosphérique. Un système dynamique est dit uniquement ergodique lorsqu'il n'admet qu'une mesure invariante, une à proportionnalité près (voir $[\mathbf{W}]$, $[\mathbf{B o w}-\mathbf{M}]$ pour les feuilletages). Pour le feuilletage horosphérique associé aux groupes convexecocompacts, le corollaire de la proposition ci-dessous affirme son unique ergodicité, en restriction comme il se doit au relevé de l'ensemble limite - le complémentaire de ce dernier fournit des mesures invariantes triviales (recensées dans le corollaire 6.5). Quoique ce résultat fût déjà bien connu (voir [Bow-M], et pour certains cas particuliers $[\mathbf{F u r}]$ et $[\mathbf{B u}])$, nous avons jugé utile de détailler une démonstration nouvelle — et courte - de ce cas particulier du théorème 6.4 subséquent, lequel semble en revanche nouveau et constitue l'aboutissement de ce chapitre. La preuve générale de ce dernier comportant nécessairement quelques complications supplémentaires, nous avons ainsi espéré que le lecteur trouverait ci-dessous un exposé plus clair de la démarche de fond, auquel d'ailleurs il sera constamment fait référence par la suite afin d'éviter un excès de redites.

Pour un groupe convexe-cocompact, en mettant en ligne de compte l'espèce de continuité des moyennes horosphériques qu'indique le lemme 1.16, il s'avère que la convergence établie au théorème 6.1 a en fait lieu partout (au-dessus de l'ensemble 
limite) : c'est la proposition suivante; la condition technique exigeant que le bord des boules instables soit négligeable est acquise en courbure constante (voir [Ro2], proposition 3.1), tandis qu'autrement il est toujours possible de l'éluder à l'aide de fonctions plateaux. Or c'est un principe général que la convergence en tout point de moyennes ergodiques signifie l'unique ergodicité (voir $[\mathbf{W}]$ ), et le corollaire ci-dessous le mettra en œuvre.

Proposition 6.2. - Supposons que $\Gamma$ soit convexe-cocompact. Soit $\mu$ l'unique densité conforme de dimension $\delta(\Gamma)$, invariante par $\Gamma$ et normalisée, et soit $m_{\Gamma}$ la mesure de Bowen-Margulis-Sullivan associée. Supposons en outre que pour tout $u \in S X$, l'on ait $\mu_{H^{+}(u)}\left(\partial B^{+}(u, 1)\right)=0$. Alors, pour toute fonction $h \in C(S X / \Gamma)$ que l'on relève en une fonction $\widetilde{h}$ sur $S X$, et pour tout $u \in S X$ tel que $g^{-\infty} u \in \Lambda(\Gamma)$ (autrement dit pour toute horosphère $H^{+}(u)$ basée dans $\Lambda(\Gamma)$ ), la moyenne

$$
\frac{1}{\mu_{H^{+}(u)}\left(B^{+}(u, r)\right)} \int_{B^{+}(u, r)} \tilde{h} d \mu_{H^{+}(u)}
$$

converge vers

$$
\frac{1}{\left\|m_{\Gamma}\right\|} \int h d m_{\Gamma}
$$

quand $r \rightarrow+\infty$ (la convergence est même uniforme sur le support de $m$ ).

Corollaire. - La mesure $\widehat{\mu}$ est, à normalisation près, l'unique mesure de Radon sur $\Lambda(\Gamma) \times \mathbb{R} \subset \mathcal{H}$ invariante par $\Gamma$.

Démonstration. - Notons $\mathcal{E}$ l'ensemble des points de $S X$ à extrémités dans $\Lambda(\Gamma)$. Par hypothèse, le groupe $\Gamma$ agit de façon cocompacte sur $\mathcal{E}$. Il revient au même, dans les conditions de l'énoncé, de prouver le résultat pour les fonctions caractéristiques des boréliens bornés de bord $m_{\Gamma}$-négligeable à la place des fonctions continues. Aussi considérons $A \subset S X$, borné et de bord $m$-négligeable. Prenons $\varepsilon>0$.

Grâce au lemme 1.16 et à la condition technique de l'énoncé, l'on vérifie que l'application $(u, r) \mapsto \mu_{H^{+}(u)}\left(B^{+}(u, r)\right)$ est continue sur $\mathcal{E} \times \mathbb{R}^{+*}$ (on utilise aussi l'action du flot géodésique pour voir que les boules de tout rayon sont de bord négligeable); elle est de plus invariante par $\Gamma$ et strictement positive sur cet ensemble. Par conséquent, on peut trouver $\alpha \in] 0, \varepsilon / 4$ [ fixé assez petit de sorte que, pour tout $u$ dans $\mathcal{E}$, l'on ait

$$
\mu_{H^{+}(u)}\left(B^{+}\left(u, e^{\alpha}\right)\right) \leqslant e^{\varepsilon / 4} \mu_{H^{+}(u)}\left(B^{+}\left(u, e^{-\alpha}\right)\right) .
$$

Comme $A$ est de bord négligeable, on peut trouver $C_{\alpha} \supset A_{\alpha}$ et $C_{-\alpha} \subset A_{-\alpha}$ tous deux de bord $m$-négligeable (la notation $A_{ \pm \alpha}$ a été introduite au paragraphe $1 \mathrm{H}$ ), et tels que $m\left(C_{\alpha}\right)-\varepsilon\left\|m_{\Gamma}\right\| \leqslant m(A) \leqslant m\left(C_{-\alpha}\right)+\varepsilon\left\|m_{\Gamma}\right\|$ pourvu que $\alpha$ soit assez petit, ce que l'on supposera dorénavant.

Prenons maintenant $r_{1}, r_{2}, r_{3}>0$ assez petits afin que la conclusion du lemme 1.16 soit valide pour la mesure $m$, avec $\alpha$ à la place de $\varepsilon$, et 1 à la place de $r$. Comme $\mathcal{E} / \Gamma$ 
est compact, on peut trouver un ensemble fini $F \subset \mathcal{E}$ tel que $\mathcal{E}$ est recouvert par les cellules $C\left(u ; r_{1}, r_{2}, r_{3}\right), u$ décrivant $\Gamma F$.

Considérons $u \in \Gamma F, v \in C\left(u ; r_{1}, r_{2}, r_{3}\right)$ et $t \geqslant 0$. En appliquant deux fois le lemme 1.16 (une fois avec $S X$ à la place de $A$ ), et grâce au choix de $\alpha$, on obtient l'encadrement suivant :

$$
\begin{aligned}
e^{-3 \varepsilon / 4} \sum_{\gamma \in \Gamma} \frac{\mu_{H^{+}(u)}\left(B^{+}\left(u, e^{-\alpha}\right) \cap g^{-t} \gamma A_{-\alpha}\right)}{\mu_{H^{+}(u)}\left(B^{+}\left(u, e^{-\alpha}\right)\right)} & \leqslant \sum_{\gamma \in \Gamma} \frac{\mu_{H^{+}(v)}\left(B^{+}(v, 1) \cap g^{-t} \gamma A\right)}{\mu_{H^{+}(v)}\left(B^{+}(v, 1)\right)} \\
& \leqslant e^{3 \varepsilon / 4} \sum_{\gamma \in \Gamma} \frac{\mu_{H^{+}(u)}\left(B^{+}\left(u, e^{\alpha}\right) \cap g^{-t} \gamma A_{\alpha}\right)}{\mu_{H^{+}(u)}\left(B^{+}\left(u, e^{\alpha}\right)\right)} .
\end{aligned}
$$

Par le corollaire 3.2, on a, dès que $t$ est assez grand, pour tout $u \in \Gamma F$ ( $F$ étant fini),

$$
e^{-\varepsilon / 4} \frac{m\left(C_{-\alpha}\right)}{\left\|m_{\Gamma}\right\|} \leqslant \sum_{\gamma \in \Gamma} \frac{\mu_{H^{+}(u)}\left(B^{+}\left(u, e^{ \pm \alpha}\right) \cap g^{-t} \gamma C_{ \pm \alpha}\right)}{\mu_{H^{+}(u)}\left(B^{+}\left(u, e^{ \pm \alpha}\right)\right)} \leqslant e^{\varepsilon / 4} \frac{m\left(C_{\alpha}\right)}{\left\|m_{\Gamma}\right\|} .
$$

Il en résulte que pour tout $v \in \mathcal{E}$ et $t$ assez grand,

$$
e^{-\varepsilon}\left(\frac{m(A)}{\left\|m_{\Gamma}\right\|}-\varepsilon\right) \leqslant \sum_{\gamma \in \Gamma} \frac{\mu_{H^{+}(v)}\left(B^{+}(v, 1) \cap g^{-t} \gamma A\right)}{\mu_{H^{+}(v)}\left(B^{+}(v, 1)\right)} \leqslant e^{\varepsilon}\left(\frac{m(A)}{\left\|m_{\Gamma}\right\|}+\varepsilon\right) .
$$

On en conclut, en faisant tendre $\varepsilon$ vers 0 , que

$$
\sum_{\gamma \in \Gamma} \frac{\mu_{H^{+}(v)}\left(B^{+}(v, 1) \cap g^{-t} \gamma A\right)}{\mu_{H^{+}(v)}\left(B^{+}(v, 1)\right)}
$$

converge vers $m(A) /\left\|m_{\Gamma}\right\|$ quand $t \rightarrow+\infty$, uniformément pour $v \in \mathcal{E}$. Or $\mathcal{E}$ est invariant par le flot géodésique et la moyenne ci-dessus au point $v=g^{-t} u$ vaut

$$
\sum_{\gamma \in \Gamma} \frac{\mu_{H^{+}(u)}\left(B^{+}\left(u, e^{t}\right) \cap \gamma A\right)}{\mu_{H^{+}(u)}\left(B^{+}\left(u, e^{t}\right)\right)} .
$$

Ceci achève la preuve de la proposition.

Démontrons à présent le corollaire. Soit $\nu$ une mesure de Radon sur $\Lambda(\Gamma) \times \mathbb{R}$ non nulle et invariante par $\Gamma$. Il suffit de considérer le cas où $\nu$ est ergodique pour l'action de $\Gamma$.

Commençons par introduire une mesure de Radon $M$ sur $S X$ construite à partir de $\nu$, ayant même rapport avec cette dernière que $m$ avec $\widehat{\mu}$ (voir $1 \mathrm{C}$ et $1 \mathrm{D}$ ). En identifiant $S X$ à $\partial^{2} X \times \mathbb{R}$, définissons la mesure $M$ par

$$
d M(u)=d \nu(\xi, t) e^{\delta \beta_{\eta}(x, u)} d \mu_{x}(\eta) \quad \text { où } u=(\xi, \eta, t) \in \partial^{2} X \times \mathbb{R} .
$$

On constate aisément que cette expression est indépendante du choix du point $x \in X$, et que $M$ est invariante par $\Gamma$. Il est clair que le support de $M$ est $\mathcal{E}$. Comme $\mathcal{E} / \Gamma$ est compact, la mesure quotient $M_{\Gamma}$ est finie. Observons que pour $\nu=\widehat{\mu}$, on a $M=m$, et réciproquement parce que $\mu_{o}$ n'a pas d'atomes ( $c f$. corollaire 1.8). 
Il est facile de voir que $M$ conserve partiellement la structure de produit local que possède $m$ (voir proposition 1.12). En désignant par $H^{+}$l'horosphère instable dans $S X$ déterminée par une horosphère $H$ de $\mathcal{H}$, on a, pour toute fonction borélienne positive $h$, l'identité suivante :

$$
\int_{S X} h d M=\int_{\mathcal{H}} d \nu(H) \int_{H^{+}} d \mu_{H^{+}} h .
$$

Cette structure suffit à garantir la propriété d'auto-adjonction des moyennes sur les boules instables par rapport à $M$, que nous connaissons déjà pour $m$ par le lemme qui referme la preuve du théorème 6.1 , auquel le lecteur voudra bien se rapporter. La preuve s'en maintient à l'identique, et nous pouvons affirmer que l'expression suivante est symétrique en les fonctions $h$ et $\psi$ boréliennes positives :

$$
\int d M_{\Gamma}(u) \varphi(u) \int_{B^{+}(u, r)} \widetilde{\psi}(v) d \mu_{H^{+}(u)}(v) .
$$

Considérons $h \in C(S X / \Gamma)$ positive. En utilisant la propriété décrite à l'instant, on obtient l'identité suivante, pour $r>0$ :

$$
\begin{aligned}
\int d M_{\Gamma}(u) \frac{1}{\mu_{H^{+}(u)}\left(B^{+}(u, r)\right)} \int_{B^{+}(u, r)} & \tilde{h} d \mu_{H^{+}(u)} \\
= & \int d M_{\Gamma}(u) h(u) \int_{B^{+}(u, r)} \frac{d \mu_{H^{+}(u)}(v)}{\mu_{H^{+}(u)}\left(B^{+}(v, r)\right)} .
\end{aligned}
$$

Notons

$$
\chi(u, r)=\int_{B^{+}(u, r)} \frac{d \mu_{H^{+}(u)}(v)}{\mu_{H^{+}(u)}\left(B^{+}(v, r)\right)} .
$$

Il est clair que $\chi(., r)$ est invariante par $\Gamma$ et continue, et que $\chi(u, r)=\chi\left(g^{-\log r} u, 1\right)$. Comme $\Gamma$ agit de façon cocompacte sur $\mathcal{E}$ qui est invariant par le flot géodésique, il existe une constante $C_{0}$ telle que $\chi(., 1) \leqslant C_{0}$ sur $\mathcal{E}$, et par suite $\chi \leqslant C_{0}$ sur $\mathcal{E} \times \mathbb{R}^{+*}$. Ainsi le second membre de l'identité ci-dessus est-il majoré par $C_{0} \int \varphi d M_{\Gamma}$.

Quant au premier membre, en vertu de la proposition précédente, on sait qu'il tend vers

$$
\frac{\left\|M_{\Gamma}\right\|}{\left\|m_{\Gamma}\right\|} \int \varphi d M_{\Gamma}
$$

quand $r \rightarrow+\infty$.

D'où il ressort que $m_{\Gamma} \leqslant C M_{\Gamma}$ pour une constante $C$. On en déduit que $\widehat{\mu}$ est absolument continue par rapport à $\nu$, en faisant entrer en ligne de compte la nonatomicité de $\mu$. La dérivée de Radon-Nikodym $d \widehat{\mu} / d \nu$ est essentiellement invariante par $\Gamma$, et par suite constante, $\nu$ étant ergodique pour l'action de $\Gamma$. Ainsi se termine la preuve du corollaire. 
À présent que nous avons achevé ce point particulièrement destiné à éclairer notre démarche, nous nous tournons vers le théorème général d'unique ergodicité du feuilletage horosphérique (théorème 6.4) en restriction aux horosphères basées dans l'ensemble limite conique - lequel constituait dans le cas précédent l'ensemble limite tout entier.

Nous devons toutefois commencer par décrire une légère condition technique $((*)$ ci-dessous) que nécessitera la démonstration plus loin, et que nous n'avons pu réduire dans notre cadre général des espaces $\mathrm{CAT}(-1)$; elle est cependant vérifiée dans pour ainsi dire la presque totalité des exemples, ce qu'enregistre la proposition 6.3. Nous noterons dorénavant $\mathcal{C}$ l'ensemble des éléments de $S X$ dont les extrémités sont dans $\Lambda_{c}(\Gamma)$. Notre condition s'énonce ainsi :

(*) Il existe un entier $N>1$ tel que pour tout $u \in \mathcal{C}$, pour tout $r>0$, on peut trouver $u_{i} \in S X(i=1, \ldots, N)$ tels que $B^{+}(u, r) \cap \overline{\mathcal{C}} \subset \bigcup_{i=1}^{N} B^{+}\left(u_{i}, r / 2\right)$.

Proposition 6.3. - La condition $(*)$ est remplie dans les cas suivants.

(a) $X$ est une variété à courbure pincée.

(b) $X$ est une surface (sans borne inférieure sur la courbure).

(c) Il existe un groupe discret d'isométries convexe-cocompact dont l'ensemble limite contient celui de $\Gamma$.

Le cas (c) comprend deux sous-cas notables :

(c1) X admet un groupe discret d'isométries cocompact.

(c2) $\Gamma$ est convexe-cocompact.

Démonstration. - Le cas (b) est trivial (avec $N=3$ ).

Pour le reste, on commence par se convaincre que $(*)$ est vraie dès qu'il existe un $N$ tel que toute boule instable de base dans $\Lambda_{c}(\Gamma)$ et de rayon $r$ ne contienne pas plus de $N$ boules disjointes de centres dans $\overline{\mathcal{C}}$ et de rayon $\lambda r$, pour un certain $\lambda \in] 0,1[$ (dans ce qui suit, $N$ et $\lambda$ varient d'une étape à l'autre). Puis, en faisant agir le flot géodésique (par contraction) et en se rappelant la définition des points limites coniques, on voit qu'il suffit de montrer que toute boule instable de base dans $\Lambda(\Gamma)$, de centre dans un certain compact de $S X$ et de rayon $r \leqslant 1$ ne contient pas plus de $N$ boules disjointes de centres dans $\overline{\mathcal{C}}$ et de rayon $\lambda r$. Enfin, après projection à l'infini, eu égard aux rapports qu'entretiennent les distances horosphériques et les distances visuelles (confer paragraphe $1 \mathrm{G}$ ), le problème revient à montrer que toute boule visuelle centrée dans $\Lambda(\Gamma)$ et de rayon $\rho$ ne contient pas plus de $N$ boules de rayon $\lambda \rho$.

À présent, dans le cas (c), le groupe convexe-cocompact dont on dispose fournit une mesure de Patterson-Sullivan qui donne à toute boule visuelle centrée en son ensemble limite (également conique) et de rayon $\rho$ une masse proportionnelle à $\rho^{D}$ où $D$ est son exposant critique - ce d'après le lemme de l'ombre 1.3. La propriété désirée s'ensuit. 
Pour finir, dans le cas (a), une boule visuelle de rayon $\rho$ contenant $N$ boules de rayon $\lambda \rho$ peut être vue, à dilatation bornée près, comme l'ombre d'une boule de $X$ de rayon 1 contenant elle-même $N$ boules de rayon $\lambda^{\prime}$, pour un certain $\left.\lambda^{\prime} \in\right] 0,1[$ ne dépendant que de $\lambda$ et du pincement de la courbure; le théorème de comparaison de Rauch emporte alors la conclusion voulue.

Dans [Rat] et [Dan], a été établie une classification des probabilités invariantes par le flot horocyclique sur une surface géométriquement finie de courbure constante (c'est à quoi se réduit dans notre cadre ces travaux situés dans celui des groupes de Lie), qui généralise le résultat d'unique ergodicité concernant les groupes cocompacts ; en restreignant l'espace des horocycles à ceux qui sont basés dans l'ensemble limite conique - qui forme dans le cas considéré l'ensemble limite privé des points paraboliques -, on retrouve une unique ergodicité. L'objet du théorème 6.4 ci-dessous est d'étendre ce dernier résultat pour tous les groupes admettant une mesure de BMS finie dans un espace CAT $(-1)$ : le feuilletage horosphérique restreint à $\Lambda_{c}(\Gamma) \times \mathbb{R}$ est uniquement ergodique. Notons que les mesures invariantes infinies y sont envisagées au même titre que les mesures finies. La preuve reprend le départ de celle de la proposition 6.2, à laquelle le lecteur sera fréquemment renvoyé, mais il ne saurait être question cette fois d'établir un résultat de convergence de toutes les moyennes horosphériques : les boules instables fournissant des moyennes convergentes ne pourront être que celles dont le rayon est de l'ordre de l'exponentielle d'un temps de retour dans un compact de son centre selon le flot géodésique (voir première étape) ; il sera toutefois possible de retrouver in fine (quatrième étape) le fil de la preuve du corollaire de la proposition 6.2 .

THÉORÈmE 6.4. - Supposons que $\Gamma$ admette une mesure de Bowen-Margulis-Sullivan finie, associée à une densité $\mu$ conforme de dimension $\delta=\delta(\Gamma)$ et invariante par $\Gamma$, et que la condition $(*)$ soit remplie. Alors $\widehat{\mu}$ est, à normalisation près, l'unique mesure de Radon sur $\mathcal{H}$ invariante par $\Gamma$ et portée $\operatorname{par} \Lambda_{c}(\Gamma) \times \mathbb{R}$.

Le théorème précédent conduit à une classification complète des mesures invariantes par le feuilletage horosphérique pourvu que l'on connaisse suffisamment bien les points de $\Lambda(\Gamma) \backslash \Lambda_{c}(\Gamma)$, ne serait-ce déjà lorsque cet ensemble est dénombrable. C'est le cas lorsque $\Gamma$ est en outre géométriquement fini, au vu de la définition que nous avons redonnée en $1 \mathrm{~F}$, et la classification complémentaire des mesures invariantes ergodiques sur $\left(\partial X \backslash \Lambda_{c}(\Gamma)\right) \times \mathbb{R}$ s'obtient aisément (ce qu'il faut connaître de l'action d'un sous-groupe parabolique borné est indiqué par le lemme 1.9). Le corollaire ci-dessous dresse donc cette classification des mesures invariantes par le feuilletage horosphérique lorsque $\Gamma$ est géométriquement fini. Dans le cas du disque hyperbolique, l'on retrouve les résultats de $[\mathbf{R a t}]$ et $[\mathbf{D a n}]$, avec ce supplément que les mesures infinies ont été considérées; or dès que $\delta(\Gamma)$ n'est pas maximal, la mesure invariante par 
le flot horocyclique attachée à $\widehat{\mu}$ est $m^{\mu, \lambda}$ où $\lambda$ est la densité de Lebesgue (voir 1C), qui est éminemment infinie (vu sa quasi-invariance par le flot géodésique, voir 1C).

Corollaire 6.5. - Supposons que $\Gamma$ soit géométriquement fini et admette une mesure de Bowen-Margulis-Sullivan finie associée à une densité $\mu$ conforme de dimension $\delta=\delta(\Gamma)$ et invariante par $\Gamma$, et que $(*)$ soit remplie. Alors toute mesure de Radon sur l'espace des horosphères $\mathcal{H}=\partial X \times \mathbb{R}$ invariante par $\Gamma$ et ergodique pour l'action de $\Gamma$ sur $\mathcal{H}$ appartient à l'une des familles suivantes, à une constante de proportionnalité près :

(a) Les mesures du type $\sum_{\gamma \in \Gamma} \mathcal{D}_{\gamma H}$ où $H \in \mathcal{H}$ est une horosphère basée hors de $\Lambda(\Gamma)$ et où $\mathcal{D}_{\gamma H}$ désigne la masse de Dirac au point $\gamma H$ de $\mathcal{H}$.

(b) Les mesures du type $\sum_{\gamma \in \Gamma / \Pi} \mathcal{D}_{\gamma H}$ où $H \in \mathcal{H}$ est une horosphère basée en un point fixe parabolique de $\Gamma$, et où $\Gamma / \Pi$ désigne un système de représentants des classes $\grave{a}$ gauche de $\Gamma$ selon le sous-groupe parabolique maximal $\Pi$ stabilisant ledit point.

(c) La mesure $\widehat{\mu}$.

Démonstration. - Soit $\nu$ une mesure de Radon sur $\Lambda_{c}(\Gamma) \times \mathbb{R}$, non nulle et invariante par $\Gamma$. Il suffit de considérer le cas où $\nu$ est ergodique pour l'action de $\Gamma$. En procédant comme dans la preuve du corollaire de la proposition 6.2 , à laquelle nous renverrons largement le lecteur, on construit une mesure de Radon $M$ sur $S X$, portée par l'ensemble $\mathcal{C}$ des points à extrémités dans $\Lambda_{c}(\Gamma)$ et invariante par $\Gamma$, donnée par la formule (indépendante du choix de $x) d M(u)=d \nu(\xi, t) e^{\delta \beta_{\eta}(x, u)} d \mu_{x}(\eta)$ où $u=(\xi, \eta, t) \in \partial^{2} X \times \mathbb{R}$.

Comme à l'endroit cité, on vérifie alors que l'expression

$$
\int d M_{\Gamma}(u) \varphi(u) \int_{B^{+}(u, r)} \widetilde{\psi}(v) d \mu_{H^{+}(u)}(v)
$$

est symétrique en les fonctions $h$ et $\psi$ boréliennes positives. Nous emploierons cette propriété fondamentale d'auto-adjonction notamment pour achever la présente démonstration comme a été conduite la preuve citée (quatrième étape plus loin). D'ici là, il nous faudra dégager une propriété de convergence des moyennes horosphériques qui restitue une part suffisante de la proposition 6.2. Le comportement de la moyenne sur une boule $B^{+}(u, r)$ paraitra convenable pour un rayon $r$ dépendant de $u$ (première étape) puis, à l'aide d'un argument de recouvrement de Vitali, pour $u$ dans un ensemble $\Omega_{r}$ associé à chaque rayon $r$ (seconde étape), lequel ensemble se révélera assez gros pour au moins une valeur de $r$ (troisième étape).

Choisissons une fonction positive $\rho \in C_{c}(S X / \Gamma)$ à support assez large pour que l'on ait $\int \rho d M_{\Gamma}=1$ et $\int \rho d m_{\Gamma}>0$. La fonction $\rho$ se relève sur $S X$ en $\widetilde{\rho}$. Introduisons la mesure $P=\widetilde{\rho} M$ sur $S X$, qui se projette sur $S X / \Gamma$ en une probabilité. Il est temps de faire remarquer que $M$ peut être a priori infinie, et c'est justement pourquoi nous avons besoin d'une telle fonction $\rho$. 
Pour $R>0$, notons $K_{R}$ le compact $S \overline{B(o, R)} \cap \overline{\mathcal{C}}$, et considérons l'ensemble $\mathcal{C}_{R}$ des points $u \in S X$ pour lesquels il existe une suite de réels $t_{i}$ tendant vers $+\infty$ avec $i$ telle que $g^{-t_{i}} u \in \Gamma K_{R}$ pour tout $i$. Avec la définition de $\Lambda_{c}(\Gamma)$, on voit que $\mathcal{C}$ est la réunion croissante des $\mathcal{C}_{R}$ quand $R$ croît indéfiniment. Puisque $P_{\Gamma}(\mathcal{C} / \Gamma)=1$, fixons un $R>0$ assez grand pour que l'on ait $P_{\Gamma}\left(\mathcal{C}_{R} / \Gamma\right)>1-1 / 16 N$ où $N$ est donné par la condition $(*)$.

Signalons que dans toute la suite, les symboles $c_{1}, c_{2}, \ldots$ désigneront des constantes strictement positives ne dépendant que des données précédentes et pas de celles à venir.

Considérons une fonction positive $h \in C_{c}(S X / \Gamma)$ fixée. La première étape cidessous exprime l'essentiel (en ne retenant que le sens des inégalités qui nous sera utile) de ce qui subsiste ici de l'estimation des moyennes horosphériques à partir du mélange du flot géodésique (corollaire 3.2) suivant le cheminement de la preuve de la proposition 6.2. La seconde inégalité ci-dessous est nécessaire à la mise en œuvre de l'argument de type Vitali de la seconde étape.

Première étape. - Pour tout $u \in \mathcal{C}_{R}$, il existe $r=r(u)>0$ tel que

$$
\begin{aligned}
\int_{B^{+}(u, r)} \tilde{h} d \mu_{H^{+}(u)} & \geqslant c_{1} \int \varphi d m_{\Gamma} \int_{B^{+}(u, r)} \tilde{\rho} d \mu_{H^{+}(u)} \\
\text { et } \quad 0<\int_{B^{+}(u, 3 r)} \tilde{\rho} d \mu_{H^{+}(u)} & \leqslant c_{2} \int_{B^{+}(u, r)} \tilde{\rho} d \mu_{H^{+}(u)} .
\end{aligned}
$$

Démonstration. - Fixons $\varepsilon \in] 0, \log 2[$ assez petit afin que

$$
\int h_{-\varepsilon} d m_{\Gamma} \geqslant \frac{1}{2} \int \varphi d m_{\Gamma} \text { et } \int \rho_{-\varepsilon} d m_{\Gamma} \geqslant \frac{1}{2} \int \rho d m_{\Gamma}
$$

d'après la notation $\widetilde{h}_{-\varepsilon}$ introduite au paragraphe $1 \mathrm{H}$. Prenons alors $r_{1}, r_{2}, r_{3}>0$ assez petits afin que la conclusion du lemme 1.16 soit valable pour les valeurs 1 et 3 de $r$. On peut trouver un ensemble fini $F \subset K_{R}$ tel que $K_{R}$ soit recouvert par les cellules $C\left(u ; r_{1}, r_{2}, r_{3}\right)$ où $u$ parcourt $F$.

Considérons $u \in \Gamma F, v \in C\left(u ; r_{1}, r_{2}, r_{3}\right)$ et $t \geqslant 0$. En appliquant le lemme 1.16, on obtient les inégalités suivantes, où $\psi$ désigne aussi bien $\rho$ que $h$ :

$$
\begin{aligned}
& \frac{1}{2} \int_{B^{+}\left(u, \frac{1}{2}\right)} \widetilde{\psi}_{-\varepsilon} \circ g^{t} d \mu_{H^{+}(u)} \leqslant \int_{B^{+}(v, 1)} \widetilde{\psi} \circ g^{t} d \mu_{H^{+}(v)} \\
& \text { et } \int_{B^{+}(v, 3)} \tilde{\psi} \circ g^{t} d \mu_{H^{+}(v)} \leqslant 2 \int_{B^{+}(u, 6)} \widetilde{\psi}_{2} \circ g^{t} d \mu_{H^{+}(u)} \text {. }
\end{aligned}
$$


Par le corollaire 3.2, vu que $F$ est fini, dès que $t$ est assez grand, on a, pour tout $v \in \Gamma K_{R}$

$$
\begin{aligned}
\frac{c_{3}}{8\left\|m_{\Gamma}\right\|} \int \psi d m_{\Gamma} & \leqslant \frac{c_{3}}{4\left\|m_{\Gamma}\right\|} \int \psi_{-\varepsilon} d m_{\Gamma} \leqslant \int_{B^{+}(v, 1)} \widetilde{\psi} \circ g^{t} d \mu_{H^{+}(v)} \\
\text { et } \int_{B^{+}(v, 3)} \tilde{\psi} \circ g^{t} d \mu_{H^{+}(v)} & \leqslant \frac{4 c_{4}}{\left\|m_{\Gamma}\right\|} \int \psi_{2} d m_{\Gamma}
\end{aligned}
$$

où

$$
c_{3}=\inf _{u \in K_{R}} \mu_{H^{+}(u)}\left(B^{+}(u, 1 / 2)\right) \quad \text { et } \quad c_{4}=\sup _{u \in K_{R}} \mu_{H^{+}(u)}\left(B^{+}(u, 6)\right) .
$$

On vérifie aisément que $c_{3}>0$ et $c_{4}<\infty$ en utilisant encore le lemme 1.16 et la compacité de $K_{R}$, qui de plus est contenu dans le support $\overline{\mathcal{C}}$ de $m$.

En se rappelant le mode d'action du flot géodésique sur les boules instables et leurs mesures (voir $1 \mathrm{G}$ ), on tire de ce qui précède que pour $t$ assez grand, pour tout $u \in g^{t} \Gamma K_{R}$,

$$
\begin{aligned}
\int_{B^{+}\left(u, e^{t}\right)} \tilde{h} d \mu_{H^{+}(u)} & \geqslant c_{1} \int h d m_{\Gamma} \int_{B^{+}\left(u, e^{t}\right)} \tilde{\rho} d \mu_{H^{+}(u)} \\
\text { et } \quad 0<\int_{B^{+}\left(u, 3 e^{t}\right)} \tilde{\rho} d \mu_{H^{+}(u)} & \leqslant c_{2} \int_{B^{+}\left(u, e^{t}\right)} \tilde{\rho} d \mu_{H^{+}(u)},
\end{aligned}
$$

avec

$$
c_{1}^{-1}=\frac{32 c_{4}}{c_{3}} \int \rho_{2} d m_{\Gamma} \quad \text { et } \quad c_{2}^{-1}=c_{1} \int \rho d m_{\Gamma} .
$$

Comme pour chaque $u \in \mathcal{C}_{R}$, on dispose d'un $t=t(u)$ tel que $u \in g^{t} \Gamma K_{R}$, nous avons établi l'énoncé de la première étape, avec $r=e^{t}$.

Dans la seconde étape, nous allons recouvrir une portion suffisante de certaines boules instables de rayon $r$ donné par les boules $B^{+}(u, r(u))$ de la première étape, la seconde inégalité que nous y avons établie nous garantissant l'obtention d'un recouvrement de type Vitali ; on pourra ainsi étendre la première inégalité à des boules dont on verra lors de la troisième étape que la quantité est suffisante pour au moins un rayon $r$. Signalons que des recouvrements de Vitali sur les horosphères avaient déjà été utilisés dans $[\mathbf{R u}]$, dans le but de montrer le mélange fort du flot géodésique à partir du mélange faible.

Pour $s>0$, considérons l'ensemble $E_{s}$ des $u \in \mathcal{C}_{R}$ pour lesquels $r(u) \leqslant s$. Comme $\mathcal{C}_{R}$ est la réunion croissante des $E_{s}$ quand $s$ croît vers l'infini, et que $P_{\Gamma}\left(\mathcal{C}_{R} / \Gamma\right)>$ $1-1 / 16 N$, nous pouvons fixer un $s$ et un fermé $E \subset E_{s}$ tels que $P_{\Gamma}(E / \Gamma)>1-1 / 8 N$.

Pour $r \geqslant 0$, définissons $\Delta_{r}$ comme l'ensemble des $u \in S X$ tels que

$$
\int_{B^{+}(u, r)} \tilde{\rho} \mathbb{1}_{E} d \mu_{H^{+}(u)} \leqslant \frac{1}{2} \int_{B^{+}(u, r)} \tilde{\rho} d \mu_{H^{+}(u)} .
$$

En termes vagues, $\Delta_{r}$ est le lieu des points $u$ tels que la portion de $B^{+}(u, r)$ couverte par $E$ est insuffisante (en deçà de la moitié). 
Introduisons également l'ensemble $Z_{r}$ des points $u \in S X$ tels que

$$
\int_{B^{+}(u, r+s)} \tilde{h} d \mu_{H^{+}(u)}>2 \int_{B^{+}(u, r)} \tilde{h} d \mu_{H^{+}(u)} .
$$

Il s'agit là des points $u$ où le poids de la couronne de largeur $s$ bordant $B^{+}(u, r)$ l'emporte trop sur celui de la boule elle-même.

Notons enfin $\Omega_{r}=S X \backslash\left(\Delta_{r} \cup Z_{r}\right)$.

Seconde étape. - Pour tout $u \in \Omega_{r}$, on a

$$
\int_{B^{+}(u, r)} \widetilde{h} d \mu_{H^{+}(u)} \geqslant c_{5} \int h d m_{\Gamma} \int_{B^{+}(u, r)} \tilde{\rho} d \mu_{H^{+}(u)} .
$$

Démonstration. - Nous allons employer le lemme de Vitali suivant, dont la preuve nous a paru suffisamment connue pour que l'on se dispensât de la rappeler ici.

LEMme. - Soit $Q$ un espace métrique compact muni d'une mesure de probabilité $\pi$, et supposons donnée pour chaque $q \in Q$ une boule $B(q, r(q))$ telle que $\pi(B(q, 3 r(q))) \leqslant$ $c \pi(B(q, r(q)))$, où $c$ est une constante. Il existe alors une famille finie de boules $B_{i}=B\left(q_{i}, r\left(q_{i}\right)\right)$ deux à deux disjointes et telles que $\pi\left(\bigcup B_{i}\right) \geqslant 1 / c$.

Considérons $u \in \Omega_{r}$. Appliquons le lemme de Vitali ci-dessus avec $Q=\overline{B^{+}(u, r)} \cap E$, $\pi$ la mesure $\widetilde{\rho} \mu_{H^{+}(u)}$ restreinte à $Q$ et normalisée (elle n'est pas nulle parce que $\left.u \notin \Delta_{r}\right)$, et les boules $B^{+}(q, r(q))$ données par la première étape, pour lesquelles $c=c_{2}$. Nous disposons alors d'une famille finie de telles boules $B_{i}(i \in I)$ vérifiant la conclusion du lemme. Observons de plus que les $B_{i}$ sont contenues dans $B^{+}(u, r+s)$ puisque leurs rayons valent au plus $s(Q \subset E)$. On a alors successivement :

$$
\begin{aligned}
\int_{B^{+}(u, r)} \tilde{h} d \mu_{H^{+}(u)} & \geqslant \frac{1}{2} \int_{B^{+}(u, r+s)} \tilde{h} d \mu_{H^{+}(u)} \quad \text { car } u \notin Z_{r} \\
& \geqslant \frac{1}{2} \sum_{i \in I} \int_{B_{i}} \tilde{h} d \mu_{H^{+}(u)} \\
& \geqslant \frac{c_{1}}{2} \int \varphi d m_{\Gamma} \sum_{i \in I} \int_{B_{i}} \tilde{\rho} d \mu_{H^{+}(u)} \quad \text { d'après la première étape } \\
& \geqslant \frac{c_{1}}{2 c_{2}} \int \varphi d m_{\Gamma} \int_{B^{+}(u, r) \cap E} \tilde{\rho} d \mu_{H^{+}(u)} \quad \text { par le lemme de Vitali } \\
& \geqslant \frac{c_{1}}{4 c_{2}} \int \varphi d m_{\Gamma} \int_{B^{+}(u, r)} \tilde{\rho} d \mu_{H^{+}(u)} \quad \text { puisque } u \notin \Delta_{r} .
\end{aligned}
$$

Nous avons ainsi obtenu le résultat de la seconde étape, avec $c_{5}=c_{1} / 4 c_{2}$.

Troisième étape. — On a $P\left(\Omega_{r} / \Gamma\right)>1 / 2$ pour au moins un $r>0$.

Démonstration. - Nous allons d'abord montrer que $P_{\Gamma}\left(\Delta_{r} / \Gamma\right)<1 / 4$ pour $r>0$ quelconque. En utilisant la propriété d'auto-adjonction des moyennes horosphériques 
par rapport à $M$ indiquée au début de la présente démonstration, on établit l'identité ci-dessous $\left(E^{c}=S X \backslash E\right)$ :

$$
\begin{aligned}
& \int d M_{\Gamma}(u) \rho(u) \mathbb{1}_{\Delta_{r} / \Gamma}(u) \frac{1}{\int_{B^{+}(u, r)} \widetilde{\rho} d \mu_{H^{+}(u)}} \int_{B^{+}(u, r)} \widetilde{\rho} \mathbb{1}_{E^{c}} d \mu_{H^{+}(u)} \\
&=\int d M_{\Gamma}(u) \rho(u) \mathbb{1}_{E^{c} / \Gamma}(u) \int_{B^{+}(u, r)} \frac{\widetilde{\rho}(v) \mathbb{1}_{\Delta_{r}}(v)}{\int_{B^{+}(v, r)} \widetilde{\rho} d \mu_{H^{+}(u)}} d \mu_{H^{+}(u)}(v) .
\end{aligned}
$$

D'une part, pour $u \in \Delta_{r}$, on a

$$
\int_{B^{+}(u, r)} \tilde{\rho} \mathbb{1}_{E^{c}} d \mu_{H^{+}(u)}>\frac{1}{2} \int_{B^{+}(u, r)} \tilde{\rho} d \mu_{H^{+}(u)} .
$$

Aussi le premier membre de l'identité précédente est-il plus grand que $\frac{1}{2} P_{\Gamma}\left(\Delta_{r} / \Gamma\right)$.

D'autre part, pour $u \in \mathcal{C}$, on peut, en recourant à la condition $(*)$, recouvrir $B^{+}(u, r)$ par des boules $B^{+}\left(u_{i}, r / 2\right)$ en nombre au plus $N$. Or pour $v \in B^{+}\left(u_{i}, r / 2\right)$, on a $B^{+}(v, r) \supset B^{+}\left(u_{i}, r / 2\right)$ et donc

$$
\int_{B^{+}(v, r)} \tilde{\rho} d \mu_{H^{+}(u)} \geqslant \int_{B^{+}\left(u_{i}, \frac{r}{2}\right)} \tilde{\rho} d \mu_{H^{+}(u)} .
$$

Par conséquent,

$$
\int_{B^{+}(u, r)} \frac{\widetilde{\rho}(v)}{\int_{B^{+}(v, r)} \widetilde{\rho} d \mu_{H^{+}(u)}} d \mu_{H^{+}(u)}(v) \leqslant N
$$

pour $u \in \mathcal{C}$ qui, rappelons-le, porte $M$. Il en découle que le second membre de l'identité plus haut est plus petit que $N P_{\Gamma}\left(E^{c} / \Gamma\right)<1 / 8$.

Il résulte de tout cela que $P_{\Gamma}\left(\Delta_{r} / \Gamma\right)<1 / 4$ comme annoncé.

Examinons maintenant les ensembles $Z_{r}, r>0$. Pour ce faire, commençons par estimer (grossièrement) la croissance avec $r$ de $\int_{B^{+}(u, r)} \widetilde{\rho} d \mu_{H^{+}(u)}$. Rappelons (voir $1 \mathrm{G})$ que

$$
d\left(P_{H^{+}(u)}^{*} \mu_{H^{+}(u)}\right)(\xi)=e^{\delta \beta_{\xi}\left(o, P_{H^{+}(u)} \xi\right)} d \mu_{o}(\xi)
$$

et que

$$
\begin{aligned}
& d_{H^{+}(u)}\left(P_{H^{+}(u)} \xi, u\right)=e^{\frac{1}{2} \beta_{\xi}\left(o, P_{H^{+}(u)} \xi\right)} d_{u}\left(\xi, g^{+\infty} u\right) \\
& =\left[e^{\frac{1}{2} \beta_{\xi}(u, o)} d_{u}\left(\xi, g^{+\infty} u\right)\right] e^{\frac{1}{2} \beta_{\xi}\left(o, P_{H^{+}}(u) \xi\right)} .
\end{aligned}
$$

Il en ressort que, pour $u \in S X$ fixé, on a

$$
\int_{B^{+}(u, r)} \tilde{\rho} d \mu_{H^{+}(u)}=O\left(r^{2 \delta}\right)
$$

avec $r \geqslant 1$.

Considérons $u \in \mathcal{C}$ fixé. Le temps de séjour de $u$ dans les $Z_{r}, r \leqslant t$, entendons par là la quantité $\int_{0}^{t} \mathbb{1}_{Z_{r}}(u) d r$, est clairement majoré par le nombre $n(t)$ d'entiers $k \leqslant[t / s]$ 
tels que $u \in Z_{r}$ pour au moins un $r \in[k s,(k+1) s[$. Or, en revenant à la définition de $Z_{r}$, on voit aisément que

$$
\int_{B^{+}(u, t+2 s)} \tilde{\rho} d \mu_{H^{+}(u)} \geqslant c 2^{n(t)}
$$

pour $t$ assez grand, avec une constante $c$ (dépendant de $u$ mais non de $t$ ) dont la stricte positivité tient au fait que $\int_{B^{+}(u, r)} \widetilde{\rho} d \mu_{H^{+}(u)}>0$ pour $r$ assez grand, ce qui a bien lieu puisque $u \in \mathcal{C}$ et que par conséquent $H^{+}(u)$ rencontre l'intérieur du support de $\widetilde{\rho}$ intersecté avec $\overline{\mathcal{C}}$, d'après la proposition 1.5 . Avec ce qui précède, il en découle que $n(t)=O(\log t)$.

On a ainsi montré que pour tout $u \in \mathcal{C}$, on a

On en déduit que

$$
\frac{1}{t} \int_{0}^{t} \mathbb{1}_{Z_{r}}(u) d r \longrightarrow 0 \text {. }
$$

$$
\frac{1}{t} \int_{0}^{t} P_{\Gamma}\left(Z_{r} / \Gamma\right) d r \longrightarrow 0 \text { quand } t \longrightarrow+\infty
$$

vu que $P(S X \backslash \mathcal{C})=0$. On conclut que pour au moins un $r>0$, on a $P_{\Gamma}\left(Z_{r} / \Gamma\right)<1 / 4$, et finalement $P_{\Gamma}\left(\Omega_{r} / \Gamma\right)>1 / 2$.

Quatrième étape : conclusion. - De même que dans la preuve du corollaire de la proposition 6.2 , la propriété fondamentale d'auto-adjonction soulignée en début de preuve nous fournit l'identité suivante :

$$
\begin{aligned}
\int d M_{\Gamma}(u) \rho(u) \frac{1}{\int_{B^{+}(u, r)} \widetilde{\rho} d \mu_{H^{+}(u)}} \int_{B^{+}(u, r)} \widetilde{h} d \mu_{H^{+}(u)} & \\
& =\int d M_{\Gamma}(u) h(u) \int_{B^{+}(u, r)} \frac{\widetilde{\rho}(v) d \mu_{H^{+}(u)}(v)}{\int_{B^{+}(v, r)} \widetilde{\rho} d \mu_{H^{+}(u)}} .
\end{aligned}
$$

Dans le second membre ci-dessus, la majoration par $N$ du deuxième terme de l'intégrande a déjà été effectuée à l'étape précédente. Aussi ledit membre est-il plus petit que $N \int \varphi d M_{\Gamma}$.

Quant au premier membre, grâce à la seconde étape, on le sait plus grand que $c_{5} P_{\Gamma}\left(\Omega_{r} / \Gamma\right) \int \varphi d m_{\Gamma}$.

En prenant $r$ comme à la troisième étape, c'est-à-dire tel que $P_{\Gamma}\left(\Omega_{r} / \Gamma\right)>1 / 2$, on obtient, avec $c_{6}=2 N / c_{5}$, que $\int \varphi d m_{\Gamma} \leqslant c_{6} \int \varphi d M_{\Gamma}$, et ce pour toute $h \in C_{c}(S X / \Gamma)$ positive. Comme à la fin de la preuve du corollaire de la proposition 6.2, ceci entraine que $\widehat{\mu}$ est absolument continue par rapport à $\nu$ (grâce à la non-atomicité de $\mu$ ), et enfin que $\widehat{\mu}$ et $\nu$ sont proportionnelles. 



\section{BIBLIOGRAPHIE}

[A-S] J. Aaronson \& D. Sullivan - « Rational ergodicity of geodesic flows », Ergod. Th. Dynam. Sys. 4 (1984), p. 165-178.

[Anc] A. AnCONA - «Exemples de surfaces hyperboliques de type divergent, de mesures de Sullivan associées finies mais non géométriquement finies », non publié.

[Bab1] M. BABILLOT - «On the mixing property for hyperbolic systems », Israel $J$. Math. 129 (2002), p. 61-76.

[Bab2] _ _ «Points entiers et groupes discrets : de l'analyse aux systèmes dynamiques », in Rigidité, groupe fondamental et dynamique (P. Foulon, éd.), Panoramas et Synthèses, vol. 13, Société Mathématique de France, 2002, p. 1-119.

[Bab-L] M. BABillot \& F. Ledrappier - « Geodesic paths and horocycle flow on abelian cover », in Lie groups and ergodic theory. Proceedings of the international colloquium, Mumbai, India, January 4-12, 1996 (S.G. Dani, éd.), Stud. Math., Tata Inst. Fundam. Res., vol. 14, Narosa Publishing House, New Delhi, 1998, p. 1-32.

[Be-H-P] K. Belabas, S. Hersonsky \& F. Paulin - « Counting horoballs and rational geodesics », Bull. London Math. Soc. 33 (2001), no. 5, p. 606-612.

[Bou] M. Bourdon - «Structure conforme au bord et flot géodésique d'un CAT(-1)-espace», Enseign. Math. 41 (1995), p. 63-102.

[Bow1] B.H. BowDITCH - «Geometrical finiteness of hyperbolic groups», J. Funct. Anal. 113 (1993), no. 2, p. 245-317.

[Bow2] _ « Geometrical finiteness with variable negative curvature », Duke Math. J. 77 (1995), no. 1, p. 229-274.

[Bow-M] R. Bowen \& B. MARCus - «Unique ergodicity for horocycle foliations », Israel J. Math. 26 (1977), p. 43-67. 
[Bu $\quad$ M. Burger - «Horocycle flow on geometrically finite surfaces », Duke Math. J. 61 (1990), no. 3, p. 779-803.

[Cos] S. Cosentino - « Equidistribution of parabolic fixed points in the limit set of Kleinian groups », Ergod. Th. Dynam. Sys. 19 (1999), no. 6, p. 1437-1484.

[Dal] F. DAL'BO - « Topologie du feuilletage fortement stable», Ann. Inst. Fourier (Grenoble) 50 (2000), no. 3, p. 981-993.

[D-O-P] F. Dal'bo, J.-P. Otal \& M. Peigné - « Séries de Poincaré des groupes géométriquement finis », Israel J. Math. 118 (2000), p. 109-124.

[Dal-P] F. DAL'Bo \& M. Peigné - « Some negatively curved manifolds with cusps, mixing and counting », J. reine angew. Math. 497 (1998), p. 141-169.

[Dan] S.G. DANI - «Invariant measures and minimal sets of horospherical flows », Invent. Math. 64 (1981), p. 357-385.

[Fur] H. Furstenberg - «The unique ergodicity of the horocycle flow », in Recent Advances topol. Dynamics, Proc. Conf. topol. Dynamics Yale Univ. 1972, Lect. Notes in Math., vol. 318, Springer-Verlag, 1973, p. 95-115.

[G-H] E. Ghys \& P. DE LA HARPE - Sur les groupes hyperboliques d'après Mikhael Gromov, Progress in Math., vol. 83, Birkhäuser, 1990.

[H-K] A.B. HajIAn \& S. KaKUTANI - « Weakly wandering sets and invariant measures », Trans. Amer. Math. Soc. 110 (1964), p. 136-151.

[He] G.A. HedLund - «Fuchsian groups and mixtures », Ann. of Math. (2) 40 (1939), p. $370-383$.

[H-P2] S. Hersonsky \& F. Paulin - « Counting orbit points in coverings of negatively curved manifolds and Hausdorff dimension of cusp excursions », Ergod. Th. Dynam. Sys., à paraître.

[H-P1] _ « On the rigidity of discrete isometry groups of negatively curved spaces », Comment. Math. Helv. 72 (1997), p. 349-388.

[Ho] E. Hopf - Ergodentheorie, Springer, Berlin, 1937.

[Hub] H. HubeR - «Zur analytischen Theorie hyperbolischer Raumformen und Bewegungsgruppen», Math. Ann. 138 (1959), p. 1-26.

[Ka1] V. Kaimanovich - «Ergodicity of harmonic invariant measures for the geodesic flow on hyperbolic spaces », J. reine angew. Math. 455 (1994), p. $57-103$.

[Ka2] _ « Ergodic properties of the horocycle flow and classification of Fuchsian groups », J. Dyn. Control Syst. 6 (2000), no. 1, p. 21-56. 
[Ka3] , «SAT actions and ergodic properties of the horosphere foliation», in Rigidity in dynamics and geometry. Contributions from the programme Ergodic theory, geometric rigidity and number theory, Isaac Newton Institute for the Mathematical Sciences, Cambridge, UK, January 5-July 7, 2000 (M. Burger \& al., éds.), Springer, Berlin, 2002, p. 261-282.

[Kre] U. Krengel - Ergodic theorems, De Gruyter Studies in Mathematics, vol. 6, Walter de Gruyter, Berlin, New York, 1985.

[Kre-S] U. Krengel \& L. Sucheston - « On mixing in infinite measure spaces », Z. Wahrsch. Verw. Gebiete 13 (1969), p. 150-164.

[Kri] K. KRICKEBERG - « Strong mixing properties of Markov chains with infinite invariant measure», in Proc. Fifth Berkeley Symposium, Math. Stat. Probab. Vol 2, Part II, 1965, p. 431-445.

[L] S.P. LALLEY - « Renewal theorems in symbolic dynamics, with applications to geodesic flows, non euclidean tesselations and their fractal limits », Acta Math. 163 (1989), no. 1-2, p. 1-55.

[L-P] P. LAX \& R. PhiLips - « The asymptotic distribution of lattice points in Euclidean and non-Euclidean spaces », J. Funct. Anal. 46 (1982), p. 280350 .

[Marg] G.A. MARGuLIS - «Applications of ergodic theory to the investigation of manifolds of negative curvature », Functional Anal. Appl. 3 (1969), p. 335336.

[Patt1] S.J. Patterson - « The limit set of a fuchsian group », Acta Math. 136 (1976), p. 241-273.

[Patt2] - "Spectral theory and Fuchsian groups », Math. Proc. Cambridge Philos. Soc. 81 (1977), no. 1, p. 59-75.

[Patt3] _ « Lectures on measures on limit sets of Kleinian groups », in Analytical and geometrical aspects of hyperbolic space, Symp. Warwick and Durham/Engl. 1984, Lond. Math. Soc. Lect. Note Ser., vol. 111, Cambridge University Press, 1987, p. 281-323.

[Patt4] _ « On a lattice-point problem in hyperbolic space and related questions in spectral theory», Ark. Mat. 26 (1988), no. 1, p. 167-172.

[Pei] M. PeIGnÉ - « On the Bowen-Margulis measure of some discrete groups of isometries », Israel J. Math. 133 (2003), p. 77-88.

[Pol-S] M. Pollicott \& R. Sharp - « Orbit counting for some discrete groups acting on simply connected manifolds with negative curvature », Invent. Math. 117 (1994), p. 275-302. 
[Rat] M. RATNER - «Raghunathan's conjectures for $S L(2, \mathbb{R}) »$, Israel J. Math. 80 (1992), no. 1-2, p. 1-31.

[Ri-N] F. RIESZ \& B. NAGY - Lecons d'analyse fonctionnelle, Gauthier-Villars, Paris, 1965.

[Ro1] T. Roblin - «Sur la théorie ergodique des groupes discrets en géométrie hyperbolique », Thèse, Université d'Orsay.

[Ro2] _ « Sur l'ergodicité rationnelle et les propriétés ergodiques du flot géodésique dans les variétés hyperboliques », Ergod. Th. Dynam. Sys. 20 (2000), no. 6, p. 1785-1819.

[Ru] D.J. RudolPH - « Ergodic behaviour of Sullivan's geometric measure on a geometrically finite hyperbolic manifold», Ergod. Th. Dynam. Sys. 2 (1982), p. $491-512$.

[Sch] K. SchmidT - Cocycles on ergodic transformation groups, Macmillan Lectures in Mathematics, vol. 1, The Macmillan Company of India Ltd., Delhi, Bombay, Calcutta, Madras, 1977.

[Sull1] D. Sullivan - « The density at infinity of a discrete group of hyperbolic motions », Publ. Math. Inst. Hautes Études Sci. 50 (1979), p. 171-202.

[Sull2] _ «Dicrete conformal groups and measurable dynamics», Bull. Amer. Math. Soc. (N.S.) 6 (1982), p. 57-73.

[Sull3] _ « "Entropy, Hausdorff measures old and new, and limit sets of geometrically finite Kleinian groups », Acta Math. 153 (1984), p. 259-277.

[Tu] P. TUkia - « The Poincaré series and the conformal measure of conical and Myrberg limit points », J. Analyse Math. 62 (1994), p. 241-259.

[CdV] Y. Colin de VerdiÈre - «Théorie spectrale des surfaces de Riemann d'aire infinie », in Colloq. Honneur L. Schwartz, Ec. Polytech. 1983, Vol.2, Astérisque, vol. 132, Société Mathématique de France, 1985, p. 259-275.

[W] P. WALTERS - An introduction to ergodic theory, Graduate Texts in Math., vol. 79, Springer-Verlag, New York, Heidelberg, Berlin, 1982.

[Y] C.B. YUE - « The ergodic theory of discrete isometry groups of manifolds of variable negative curvature», Trans. Amer. Math. Soc. 348 (1996), no. 12, p. $4965-5005$. 